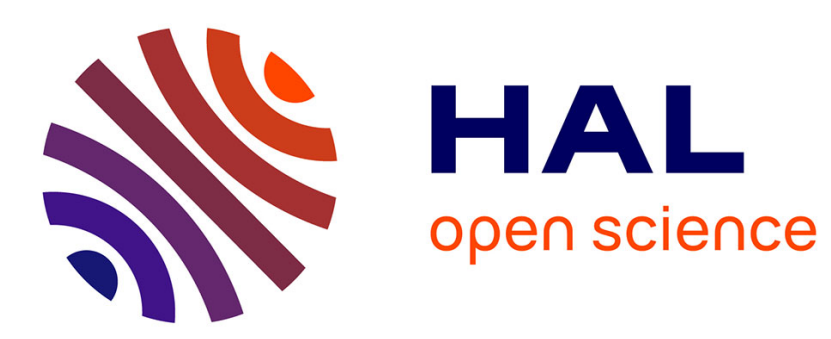

\title{
Do countries falsify economic data strategically? Some evidence that they might.
}

Tomasz Michalski, Gilles Stoltz

\section{To cite this version:}

Tomasz Michalski, Gilles Stoltz. Do countries falsify economic data strategically? Some evidence that they might.. Review of Economics and Statistics, 2013, 95 (2), pp.591-616. 10.1162/REST_a_00274 . halshs-00482106v3

\section{HAL Id: halshs-00482106 https://shs.hal.science/halshs-00482106v3}

Submitted on 15 Jul 2011

HAL is a multi-disciplinary open access archive for the deposit and dissemination of scientific research documents, whether they are published or not. The documents may come from teaching and research institutions in France or abroad, or from public or private research centers.
L'archive ouverte pluridisciplinaire HAL, est destinée au dépôt et à la diffusion de documents scientifiques de niveau recherche, publiés ou non, émanant des établissements d'enseignement et de recherche français ou étrangers, des laboratoires publics ou privés. 


\title{
DO COUNTRIES FALSIFY ECONOMIC DATA STRATEGICALLY? SOME EVIDENCE THAT THEY MIGHT.*
}

Tomasz Michalski

HEC Paris

Jouy-en-Josas, France

michalski@hec.fr
Gilles Stoltz

\author{
Ecole Normale Supérieure ${ }^{\dagger}$ CNRS \\ Paris, France \\ $\&$ \\ HEC Paris, CNRS, \\ Jouy-en-Josas, France \\ stoltz@math.ens.fr
}

July 15, 2011

\begin{abstract}
Using Benford's Law, we find evidence supporting the hypothesis that countries at times misreport their economic data strategically. We group countries with similar economic conditions and find that for countries with fixed exchange rate regimes, high negative net foreign asset positions, negative current account balances or more vulnerable to capital flow reversals we reject the first-digit law for the balance of payments data. This corroborates the intuition of a simple economic model. The main results do not seem to be driven by countries in Sub-Saharan Africa or those with low institutional quality ratings.
\end{abstract}

JEL codes: F31, F32, F33, F34, D82

Keywords: capital flows, public information provision, misinformation, Benford's Law, transparency

${ }^{*}$ We would like to thank Ai-Ting Goh, Bogumił Kaminski, Evren Örs, Martin Paldam, Romain Rancière, Tristan Tomala, Jaume Ventura, the participants at the ESSEC-HEC-INSEAD-PSE Workshop on Financial Economics and the DEGIT XV conference for helpful comments and discussions. We would like to thank two anonymous referees for their comments and suggestions. All remaining errors are ours.

${ }^{\dagger}$ This research was carried out within the INRIA project CLASSIC hosted by Ecole normale supérieure and CNRS. 


\section{Message to our readers:}

This paper is formed by two entities.

- The main text describes the results submitted for journal publication (pages 3-56).

- An appendix (Appendix C, starting on page 57) provides additional results that were not included in the journal submission. 


\section{Introduction}

Conventional wisdom has it that governments may lie strategically to the public about economic data that they collect and provide. The accusations that Greece and Italy tinkered with their budget deficit figures before joining the Euro zone provide anecdotal examples. Argentina has been suspected of understating inflation figures since mid-2007. The Hungarian government, according to its prime minister in a statement that leaked out, lied to the general public about the state of the economy to win the elections in 2006. Ukraine misreported the level of reserves to the International Monetary Fund between 1996 and 1998. China is believed to embellish its GDP growth numbers. Even the United States came under scrutiny after GDP growth revisions were consistently negative in the crisis that started in 2008. ${ }^{1}$ At times governments are caught red-handed (Hungary or Ukraine), but most of the time it is simply unclear whether the data that are provided to the public are just inaccurate (because of, say, measurement errors or bad data collection methods) or suffer from deliberate alterations. In some cases, misinforming economic agents may bring tangible (possibly short-term) gains for a government; for example, Argentina by misstating inflation figures avoided paying out higher interest on government bonds indexed to inflation (which should have constituted in fact a partial default) and raising the wages in the public sector. Greece enjoyed lower borrowing rates (close to Germany's) on its government debt because of its Euro zone membership and because investors did not know the entire extent of Greek budget troubles. Given these examples, there are two important questions to ask. Is it possible to find instances when countries or groups of countries engage in misinforming economic agents? Is there some common characteristic that is shared by these countries?

In this paper we use a statistical test based on the distribution of first digits of economic accounting data (also known as Benford's Law) to test whether countries falsify the economic figures that they report or not. Benford's distribution of the first digits arises naturally for many different types of data. This is true for processes with exponential growth or products of independent random variables; that is, in applications, for many economic data due to inflation, economic or population growth in the presence of stochastic shocks. This property is preserved under multiplication by a common factor, so will survive conversions into different currencies. This distribution arises when data from many different economic processes (from many countries) are considered together. Deviations from Benford's distribution may flag data reporting irregularities.

Our data set. In our tests we use quarterly balance of payments $(\mathrm{BOP})$ data from the International Monetary Fund (IMF) for years 1989-2007, for several reasons. ${ }^{2}$ Firstly, these accounting data are available for many countries from the same source. Secondly, there were instances where countries

\footnotetext{
${ }^{1}$ For Greece's actions before joining the Euro zone, see The Wall Street Journal (2004). For the more recent account of releasing dodgy budget statistics, see the Financial Times (2010). For Italy, see the Financial Times (2001). Argentina's story is described for example in the Financial Times (2008) and The Wall Street Journal (2008). The Hungarian case is treated in the Financial Times (2007). Ukraine's history is described inter alia in IMF (2000). For accounts for China and the USA see the Financial Times (2009).

${ }^{2}$ Section 2 details why some other statistical series of interest were not chosen for scrutiny.
} 
were either caught (Ukraine) or suspected (Russia, The Gambia, China, Chad, Sierra Leone, Egypt etc.) of misreporting this particular kind of data. Next, the first-digits of each BoP entry should by itself conform with the Benford's Law; and it should be the more so when data from multiple countries are considered (when heterogeneity is introduced). Moreover, the data are somewhat standardized (among countries and across time) as it should be prepared (categorized) by providers according to an IMF manual. They offer a lot of datapoints relatively to other data on economic fundamentals. Also, they are not transformed many times (and if the balance is recorded in U.S. dollars it is not transformed at all), something which could worsen their statistical properties. ${ }^{3}$ Moreover, as the data are quarterly, they include series that are closely watched by investors so that countries may have an incentive to misrepresent them. One would wish naturally to extend our tests to other types of macroeconomic data; but the size of the task just for the series at hand is already substantial enough.

Detailed methodology. We first advance theoretical arguments and review the literature on why the economic processes of interest should adhere to Benford's Law. Then, we show that for our entire dataset we cannot reject a Benford's distribution of the first digits. Before putting our data to a closer test, we develop a simple model (taking into account the characteristics of our data set) that gives us insights as to why and when countries would strategically misinform investors (economic agents) on the series we study. Our story is simple: a country may want to hide its true state of the world to prevent capital outflows or incite inflows. Next, we group countries in different relevant categories indicated by our model and investigate whether we can reject the hypothesis that the distribution of the first digits of data that they provide complies with Benford's distribution, which would be indicative of data manipulation.

Results. Using balance of payments data we find evidence that countries with fixed exchange rate regimes, those with high negative net foreign asset positions or negative current account balances have economic data that have irregular, non-Benford behavior of the first digits. We reject the Benford's Law for series from many groups of countries that would be more vulnerable to capital flow reversals (i.e., those with fixed exchange rates and relatively high capital openness; countries with both current and fiscal deficits). Euro-adopting countries have unusual distributions of first digits in the reported accounts after they join the Euro zone. Our main results do not seem to be driven by including countries in Sub-Saharan Africa or those with low institutional quality ratings in our samples.

Interpretation. As the rejections of the Benford distribution for the first digits of data occur for specific groups only and not for the complements in the data set (for example for fixed exchange rate regimes but not floating ones, or countries with low net foreign assets but not those with high net foreign assets), our results are consistent with the hypothesis that vulnerable countries (as indicated by our model) strategically manipulate the data. These rejections are rather category than country specific, as data from many countries for different quarters enters different categories. For example, if a country changes its exchange rate regime or the sign of the current account within the range of

\footnotetext{
${ }^{3}$ Nye and Moul (2007) found that economic series transformed many times with imprecise procedures (e.g., relying on inaccurate exchange rates) may not obey Benford's Law. They advise to work with the raw, nominal data.
} 
our sample, its data will enter different categories for different quarters. ${ }^{4}$

These rejections of the Benford's Law should not be driven by some perverse statistical features of the data. We correct for possible persistence or statistical breaks in the data by developing a specific randomization procedure, and develop its critical test values by simulations. We want our rejections of the null hypothesis to be corroborated both by traditional P-values of chi-square tests, but also with our developed tests based on selecting a fraction of the data at random that we call 1-in-5, 1-in-10, 1in-20 criteria. We group many countries sharing similar economic characteristics together so that data heterogeneity and shorter time series for more countries in each group are typically considered than in for example purely a geographical division of countries (we want to have at least 1100 observations so as to be able to use the 1-in-10 criterion). This should allow for a better adequation to Benford's Law for finite samples (see Section 5.1.2). ${ }^{5}$ The rejections for some categories (for example, among fixed vs. floating division) exist for groups comprising of a larger number of countries than the nonvulnerable groups, again favoring the strategic argument.

We doubt that the rejection of Benford's distribution for the first digits of the data for many groups of vulnerable countries according to our model is due to just poor data collection and noise in the data in those countries. Large positions in the errors and omissions section of the BoP data may indicate, of course, that the data collection process in a given country is of low quality or that there is a great amount of illicit or unrecorded transactions; but if there are no manipulations involved, the first digits of the series (even when incompletely registering transactions) should still adhere to Benford's Law (see Section C.2.1 in Appendix C). When we look at the tests based on the less persistent series, there is hardly any evidence that countries with bad institutions do have non-expected firstdigit distributions. If the rejection of Benford's distribution would be caused by bad data collection, the first-digit distribution of the less persistent series should be also affected. Moreover, robustness checks show that excluding countries in Sub-Saharan Africa or those with low institutional quality ratings as measured by World Bank WGI indicators, such as government effectiveness or rule of law, does not change our main conclusions (see Section 6.6). ${ }^{6}$ Observing various vulnerable categories, we obtain stronger results for countries with more open capital markets (which tend to be countries with better data collection metholodogies as well). Also, because we find evidence for countries that would appear (at least in the short run, according to our model) to benefit from misinforming investors, we do not think that the data suffer from rounding errors; if it were so, we would find rejections of the null hypothesis all across the board. The irregularities may not be caused merely by regular and expected interventions of authorities; for example if we exclude the item of reserves,

\footnotetext{
${ }^{4}$ We do not claim that necessarily all countries belonging to a particular group cause a discrepancy; this may be driven by few countries only. We control whether exclusion of one country at a time changes our results.

${ }^{5}$ For example, the subsets of countries that have fixed exchange rate regimes contain many countries (49 countries with 26.6 quarters on average for the stable sample and 84 countries with 32.6 quarters on average for the full sample) so that we believe based on Section 5.4.1 that the neither persistence nor structural breaks are driving our results.

${ }^{6}$ Young (2009) questions the quality of growth or price statistics provided by official sources in Sub-Saharan Africa. We exclude therefore such countries to show that they do not drive our results.
} 
which is directly impacted by central banks, we still draw the same conclusions for the first digit distributions of countries grouped by fixed and floating exchange regimes.

Relying on our evidence, which supports our economic model, we conclude that countries, at times, may falsify economic data strategically when it suits their interest. This calls for the inclusion of strategic information provision by governments in models where public signals are important.

Caveats on the practicality of our method. Unfortunately, due to data limitation of macroeconomic series (too few points per each series) our methods do not allow us to detect which country at what point provided false information. We do not know how grave the possible infractions were. Moreover, we cannot exclude that some of the observed effects would be just price tag effects, though the latter are likely to appear rather in nominal currency rather than in U.S. dollars. For example, a country might report instead of a deficit on the trade balance of 2.01 billion in a local currency a one that is slightly lower, say at 1.99 billion. Our task is complicated by the fact that countries may not cheat all of the time (as serial cheaters might be found out quickly); detecting fraud in one or two quarters when many quarters of the data are present is then unlikely. Out of necessity, we are also working with data that are sometimes revised and sent to the IMF several quarters after initial revelation. The tests cannot detect also some instances of cheating - for example if a country changes all its items by a common factor or if it changes the numbers preserving the Benford's first-digit distribution. It is also hard to envisage which digit would be favored in any potential infractions; this is because investors may possess some information of their own and the country may not stray too far away from either past values or investor expectations in order to appear credible in its announcements.

Literature review. The tests we employ are used by accounting practitioners to detect fraud in company accounts (see Nigrini [1999] for examples). Varian (1972) suggested to use Benford's Law to test the naturalness of the data and adequacy of the numbers produced by forecasting models. These tests were not used widely up to date in international (macroeconomic) comparisons; there is typically a lack of enough suitable data to boost the statistical power of such tests that render these methods impractical and until recently (for example at the time of writing of Varian [1972]) the theoretical processes generating this distribution were not well understood.

To our knowledge, there are two studies that use Benford's distribution to inspect macroeconomic data so far. Nye and Moul (2007) provide evidence (and also simulations of economic quantities) that widely used international macroeconomic data such as the GDP series in the Penn World Tables exhibits first digits that do in general conform to Benford's distribution. The data, however, do not conform to Benford's Law for non-OECD countries, posing questions about data quality; the alteration of data might come from the source (falsification or simply from an inefficient data collection infrastructure in the considered countries) or from inaccurate data transformations. Gonzalez-Garcia and Pastor (2009) follow a similar exercise and extend the analysis to annual IMF data. Their main conclusions are threefold. First, they find that most macroeconomic series they study follow Benford's Law. Second, after observing some series for individual countries - GDP for Finland and Japan - they claim that "structural breaks" in the data may lead to a failure of Benford's Law to hold in practice (as they work with a small number of observations) for countries that according to the IMF 
possess a high quality of data. ${ }^{7}$ Third, and most interestingly, they find no convincing link between independent assessments of data quality collection methods in country groups and the rejections of Benford's Law for the first digits of data, which may be consistent with our story. None of these papers, however, provides a model why countries would misreport data and do it strategically. The focus of these two papers is about assessing the quality of data used in empirical work. In these two studies countries are grouped based on geographical criteria, which in fact may be vacuous - why should all African countries be lumped together? - whereas we use groupings based on relevant economic criteria, which allows to draw some conclusions on category-specific behavior. Our groupings also should have more data heterogeneity as we consider shorter time series for more countries in each group rather than in a purely geographical division of countries. This should allow for a better adequation to Benford's Law for finite samples (see Section 5.1.2). It also appears that the indicated references do not correct for the possible persistence or structural breaks of the economic series, which could lead to false rejections of the conformity of the realized first-digit distribution with Benford's Law, while we address this issue (Gonzalez-Garcia and Pastor [2009] only notice the possibility of structural breaks for single series). We sidestep these issues by aggregating data across many countries and using a procedure to select the series at different dates to be tested randomly (see Section 5.4 for details).

From the existing literature it is clear that the public signal is important for economic agents. The research on countries' truthfulness in information provision, however, is meagre to date. The problem is that economic agents cannot readily verify whether the information issued by a government is of low quality (i.e., imprecise) or whether the government in question is supplying deliberately false information. ${ }^{8}$ Each government or statistical authority has some leeway in providing the various numbers describing the state of the economy as long as these are not too far off from the public expectations; and as long as these can be subsequently put in the errors or omissions sections. Related to our topic is the literature on public signals and transparency under strategic uncertainty recently represented inter alia by Morris and Shin (2002) or Angeletos and Pavan (2007) and explicitly in the context of a currency attack by Heinemann and Illing (2002). However, the notion of strategic investor misinformation has not been considered in this literature to our knowledge; a central authority can control at best the precision (the variance) of the public signal that the investors get, which is not the same as what we mean here. ${ }^{9}$ Note, however, that changing the precision of the signal would not constitute data manipulation and our tests would not pick it up, as the data that would be generated should still then adhere to Benford's Law (see the arguments in Section 5.1 below). Angeletos et al.

\footnotetext{
${ }^{7}$ This qualification is important; the presence of structural breaks in the data may be due to changes in data collection methodology and exactly a way to blur the measurement of some economic quantities. The discussions about the accuracy of measures of unemployment or the budget deficit under different methodologies are perennial.

${ }^{8}$ Institutions like the IMF or the Eurostat can verify at least partially the information that is given out by the country, but this does not solve the problem in its entirety.

${ }^{9} \mathrm{~A}$ different notion is that of vagueness, when a policymaker would release a set of signals containing the true one to be informative in the equilibrium; see Stein (1989) based on the model of Crawford and Sobel (1982). Gelos and Wei (2005) show empirically that funds invest less in less transparent countries.
} 
(2006) and Tarashev (2007) offer models where the government chooses a signal (the interest rate) to inform the public about the state of its economy. This affects the cost of the agent's later decision. But providing false information is a different issue; the agents may not know well the information policy of the government and (provided that the agents do pay attention to what the government says) this information may affect the belief formation in one way or the other. Extending the work of Obstfeld (1986) and Obstfeld (1996) and analyzing a second generation currency crisis model without any strategic uncertainty, Sbracia and Zaghini (2001) note (without modeling the strategic choices of a central authority) that a greater precision of public information may stop an otherwise imminent attack when the state of the economy is bad and that the contrary is true when the state is good. This opens up the question whether the government would like to use the information that is provided to its advantage. On a different note, Sandleris (2008) argues that a country may repay sovereign debt in order not to reveal bad information about the state of its economy. The literature on the strategic delay of information release is related but does not apply here. The large literature on dynamic inconsistency and limited commitment started by Kydland and Prescott (1977) is not relevant in this context; and neither is the literature on moral hazard with ex-post verifiable actions.

Successful misinformation by a government may seem puzzling and is rarely taken into account, as it appears at odds with the assumptions of rational expectations and the economic agents' knowledge about the true state of the world employed in many models. ${ }^{10}$ In some models with strategic uncertainty, information is aggregated through individual trades into prices; and hence the governments' influence over the information known to market participants may be extremely limited. This, however, may be not true all the time; for example in a fixed exchange regime where an important price - the exchange rate - is fixed, the shadow price (the real exchange rate) must be calculated based on the statistics provided by governments. A fundamental question is why individuals (knowing that the government may reveal information strategically) would pay attention to public signals at all. However the basic conclusions of the literature on one-sided private information when signalling is not possible (see for example Aumann et al. [1995], Crawford and Sobel [1982] or Benabou and Laroque [1992]) are that this can be the case, and partial information revelation may be an equilibrium. Another question is whether the ability of governments to provide false information is permanent or just temporary; in the long run serial cheaters may be found out. This paper, by providing some evidence on the strategic character of government release of public signals, provides an incentive to continue and expand theoretical research in these fields.

Organization of the paper. The paper is constructed as follows. First, we discuss the choice of our data set and then the reasons for which countries would want to misreport economic data; in Section 3 we lay out a model of financial flows and of country incentives to misinform investors

\footnotetext{
${ }^{10}$ The Billion Prices Project by Alberto Cavallo and Roberto Rigobon is an initiative by private agents to gather information in lieu of government agencies (in this case on the evolution of prices). This way of substituting the government, however, is limited by what can be observed by economic agents themselves. In the case of the balance of payments or gross domestic product data, a similar project is hard to envisage.
} 
about their state of the economy. Then in Section 4 we describe the data we use while in Section 5 we discuss the theory behind Benford's Law and the statistical methodology for our dataset. Section 6 reports the evidence and Section 7 concludes. Proofs, tables with statistical documentation and main results of our study are included in the Appendix. Other material (extended proofs, full sample results, robustness checks, etc.) is in Appendix C.

\section{Choice of a convenient data set}

We first choose a data set that would make detecting misreporting (if any occurs at all) possible given our methodology. We seek economic series which could be manipulated by the government and at the same time easily observed by investors. The indicators in question should also theoretically follow Benford's Law. For our tests to have rejection power (see Section 5) we require a lot of comparable data. Certain other characteristics are also desirable for a data set to be adequate (explained below).

For theoretical reasons, we do not expect inflation, interest rates, the fiscal deficit (as a percentage of the GDP) nor any indicators that are growth rates with some time-invariant expectation (GDP growth, changes in industrial production) to follow Benford's Law by property P1 (see Section 5), and it might be difficult to obtain enough heterogeneity so that property P2 works. In contrast, we expect that money supply, public debt (stock), budgetary entries (data in levels), output (level data) and balance of payments entries should follow the said law. Taking data from many countries (distributions) should increase adherence to the Benford's Law by property P2 (Section 5).

There are few statistics for GDP or industrial production available for each quarter for a wide range of countries and some of these are available only yearly (i.e., fiscal data). Comparable public sector debt data for most of the covered countries in the Public Sector Debt Statistics database from the World Bank/IMF are available only since 2009. For external debt in the World Bank/IMF Quarterly External Debt Statistics, more extensive quarterly coverage for countries starts with 2003-2005. ${ }^{11}$ With a small number of observations in the data sets, it is difficult to group countries according to their economic characteristics and reduce persistence by considering randomly selected subsets.

A different issue is whether the data are measured (i.e., as a flow) or artificially constructed (as an index). ${ }^{12}$ Industrial production and price levels are indices, so are not "natural" data. On the other hand, geometrically increasing sequences - like the BoP entries, fiscal data etc. - are convenient and guarantee on a theoretical side the conformity to Benford's Law. Ideally, we would like measures that are calculated using similar methodologies from country to country and that do not involve many transformations of the data. Moreover, some macroeconomic data are only one of a kind data, and so do not comprise comprehensive accounting data sets. Finally, possible persistence in the series may

\footnotetext{
${ }^{11}$ The troubling lack of availability (with speculations why such transparency could be caused by) of precise debt data even historically is highlighted for example by Reinhart and Rogoff (2009), page 137.

${ }^{12}$ Nye and Moul (2007) advise against using indices for testing the adequation to Benford's Law. Statistical methodologies used in constructing such indices and for example seasonal adjustment may worsen the "naturalness" of the data.
} 
make the rejection of the Benford's Law easier.

Data sets on the balance of payments, fiscal data and money supply satisfy most of these requirements. We chose to investigate quarterly balance of payments data from the IMF BOPS database ${ }^{13}$ for the period 1989-2007, as they had some additional advantages over other data sets. ${ }^{14}$ Available quarterly, these are accounting data containing net values, which helps counter the problem of persistence that could lead to easier rejections of the Benford distribution for the first digits in finite samples (see Section 5.4). These data are provided by statistical agencies of IMF member states and should be prepared in a standard way, using the Balance of Payments manual of the IMF (1993). The data are in U.S. dollars, which means that they were minimally transformed (for the importance of this, Nye and Moul [2007]) given the fact that most international transactions are conducted in this currency. Moreover, many countries keep track of the BoP in U.S. dollars (e.g., Poland, Romania, Taiwan, etc.). The fact that the BoP data are converted to U.S. dollars should preserve the Benford's distribution of the digits if the original distribution indeed is Benford (Section 5.1.3) but the distribution would remain non-Benford if it were such initially. Currency conversion may also help to avoid some structural breaks like currency reforms, hyperinflations or rapid disinflation that would appear in the domestic-currency data.

Why would countries manipulate the BoP data? BoP data is crucial for fixed exchange rate regimes and for countries with high net foreign liabilities. In a fixed exchange regime, the value of the information coming from the BoP is even higher to agents as the exchange rate (which would otherwise contain aggregate information about the underlying transactions) in a credible regime is expected not to change. A sustained current account imbalance may point to a misalignment of the real exchange rate that may be (abruptly) corrected by a devaluation or depreciation of the country's currency. A depletion of reserves without a possibility of borrowing liquidity may lead to a balance of payments crisis as in the literature started by Krugman (1979) or to a currency attack. Moreover, investors obtain valuable information from the BoP that helps them assessing the returns to capital (and their riskiness) within the country in the nearest future. ${ }^{15}$ For example, changes in reserves, or changes in the volume and composition of the current account items have implications for the ability to retire capital (for possible capital flow restrictions in the future), for the probability of a successful speculative attack (that would diminish the valuation of the assets held in the local currency), or for

\footnotetext{
${ }^{13}$ The data was accessed online at the IMF website on 31 December 2009.

${ }^{14}$ In further work, we analyze various fiscal data sets to uncover patterns of rejection of the Benford's Law. Most fiscal data sets contain yearly data which leads to relatively few datapoints available for a wide set of countries.

${ }^{15}$ For example, Standard and Poor's (2008) analyzes the structure of the current account, the composition of capital flows and reserve adequacy to assess a sovereign's rating. They use indices composed of different items of the BoP (balance on trade, FDI, portfolio flows, reserves, etc.) in their assessments. Imperfect as it may be, the sets of indicators published in economic press outlets may be telling what draws most attention. For example, in The Economist, the set of indices covered on a regular basis include: GDP change (\%), industrial production change ( $\%$ ), consumer prices change $(\%)$, unemployment rate (\%), trade balance (flow), current account (flow and \% of GDP), budget balance (\% of GDP) and interest rates (\%). In the IMF World Economic Outlook issues, the main indicators include output, inflation, fiscal balances, trade volumes and prices, current account, capital and financial account transactions.
} 
the solvency (or possible illiquidity) of the country and the firms located there and potential macroeconomic adjustment needed in case of a rapid correction of any current account imbalances. Reinhart and Rogoff (2009) include among the best few predictors of banking and/or currency crashes (compiling from the rich literature on early crisis indicators), respectively, the short-term capital inflows/GDP, current account balance/investment, current account balance/GDP, and exports. In the view of this, a government may want to lie on the BoP data for example to steer the market expectations to a good equilibrium (as in second generation currency crisis models), avoid capital flight or attract investment to cover a current account or a fiscal deficit.

There are several instances where countries were suspected of changing their BoP entries or outright caught doing so. ${ }^{16}$ Russia and Gambia were charged of being guilty of overstating reserves with Ukraine (see IMF [2000]) being the most blatant case. Chad and Sierra Leone were not disclosing external arrears that they ran in breach of the agreements with the IMF. China was accused of understating its trade surpluses. Analysts and commentators point in several cases to doubts about the veracity and accuracy of data (i.e., Egypt in 2005 but many other instances can be found) when the sizes of the net errors and omissions may seriously alter the current account balance shown by a country (for example, putting it into a negative territory). All these cases, except for China (the country is not in the IFS data for 1989-2007), are found in countries that were at the time of the accusations dependent on capital flows or international aid.

In the next section we develop a parsimonious stylized model where countries reveal information strategically to investors, trying to influence capital flows. The implications of this model are used to group countries for testing misreporting.

\section{Misreporting economic data to international investors: a model}

To motivate our empirical tests, we provide a parsimonious open economy model where we study the incentives of countries to provide false information to investors. We study a one shot game of incomplete information. All proofs are relegated to the Appendix. Suppose first there is a continuum of identical countries indexed $i \in[0,1]$ with a population of immobile workers $L_{i} \equiv 1$ and endowed with immobile capital installed $C_{i} \equiv C>0$. There are in total $\bar{K}$ units of mobile capital in the world; for simplicity these are owned by some agents which have no domicile, but have to invest this capital in at least one of the aforementioned countries. There are free capital flows between all countries, and perfectly competitive markets for the factors of production within each country. The remuneration of factors equals their marginal productivity.

To model a country's incentives, suppose that the news about each country's balance of payments data can be either Good $(g)$ or Bad $(b)$ reflecting the underlying state of a country's economy ${ }^{17}$; for

\footnotetext{
${ }^{16}$ For Russia: Financial Times (1999), for China: Financial Times (2003). For Chad, Gambia and Sierra Leone see the IMF press releases from 2003, 2005 and 2006 respectively. Doubts about Egypt's figures in Reuters News (2005).

${ }^{17}$ The model is stylized. The application to BoP data can be understood that good or bad data about the BoP will in-
} 
each country $i \in[0,1]$, we denote its state by $s_{i} \in\{g, b\}$. The state of the economy is modeled as a multiplicative productivity parameter and denoting by $A$ a common parameter for all countries, one has $A(g)>A(b)$. Each country has the same technology and the statistical process generating a periods' news, as well as $\Phi>0$, the present discounted value of punishment for being caught lying. ${ }^{18}$

There is only one period. For all countries, a Good state occurs with a common probability $\hat{\rho}$, which is drawn from some distribution $\rho$ on $[0,1]$. Since there is a continuum of countries, this results, by the law of large numbers, in respective fractions $\hat{\rho}$ and $1-\hat{\rho}$ of the countries being in Good and Bad states. The distribution $\rho$ is common knowledge but the actual probability $\hat{\rho}$ of a Good state is initially unknown to the countries and investors. The sequence of moves is the following. Each country $i \in[0,1]$ learns its own true state of productivity $s_{i} \in\{g, b\}$ and then declares it (truthfully or not) as $d_{i} \in\{g, b\}$ to investors simultaneously with other countries; we denote by $\tilde{\rho}$ the fraction of countries declaring a Good state. Investors observe country declarations, and then make decisions on where and how much to invest: we denote by $K_{i}\left(d_{i}, \tilde{\rho}\right)$ the total capital invested in country $i$ (including the immobile capital $C$ ). Then, the period ends, investments are realized and the payouts (including punishments, if any) are made.

We assume that the production function in country $i$,

$$
Y_{i}=A\left(s_{i}\right) F\left(K_{i}\left(d_{i}, \tilde{\rho}\right), L_{i}\right)=A\left(s_{i}\right)\left(K_{i}\left(d_{i}, \tilde{\rho}\right)\right)^{\alpha}\left(L_{i}\right)^{1-\alpha}
$$

is Cobb-Douglas with the capital intensity $0<\alpha<1$. As $L_{i}=1$ for all countries $i$, we do not analyze the model in per capita form. Let $R_{i}\left(s_{i}, d_{i}, \tilde{\rho}\right)$ be the return to a country and let $E R_{i}\left(s_{i}, d_{i}\right)=$ $\mathbb{E}\left[R_{i}\left(s_{i}, d_{i}, \tilde{\rho}\right)\right]$ be its expectation (with respect to the distribution $\rho$ ). We assume that each country $i$ cares about its gross national product (the remuneration of domestic factors), $R_{i}=w_{i} L_{i}+r_{i} C_{i}$ where $w_{i}$ is the wage and $r_{i}$ is the return to (domestically installed and owned) capital. With the above assumption on the production function, this translates into

$$
R_{i}\left(s_{i}, d_{i}, \tilde{\rho}\right)=A\left(s_{i}\right)\left((1-\alpha)\left(K_{i}\left(d_{i}, \tilde{\rho}\right)\right)^{\alpha}+\alpha\left(K_{i}\left(d_{i}, \tilde{\rho}\right)\right)^{\alpha-1} C\right) .
$$

Country $i$ 's payoff is increasing in the state of the world $s_{i}$ and in the installed (immobile) capital $C$.

We discuss only an equilibrium when a fraction of countries always lies, but investors analyze the declarations, obtain information from these and make rational investment decisions nevertheless. ${ }^{19}$

In this equilibrium, countries that receive a Good state $s_{i}=g$ report truthfully $g$; a fraction $\varphi \in$

crease/decrease potential flows if declared truthfully by a country, and the country desires (given the production functions and its objective to maximize GNP) to attract as many flows as possible. Good data about the BoP may mean that a country is less risky and offers higher returns to potential investors given the same level of flows.

${ }^{18}$ This punishment may not occur with certainty; in reality it may be very difficult for investors collectively to learn that a country misstated its information.

${ }^{19}$ The "babbling" equilibrium, as in games of cheap talk (Crawford and Sobel [1982]), where countries randomize their declarations and investors ignore the messages sent by countries does not exist here. This is because there is a positive punishment $\Phi$, so countries that would randomly lie would be punished: lying has consequences. 
$[0,1]$ of countries that receive a Bad state $s_{i}=b$ declare a Good state $g$ knowing that they will obtain in expectations a penalty $\Phi$; while the rest of those receiving the Bad state $(1-\varphi)$ declare it as such. As we show in Proposition 6 (in the Appendix), at equilibrium, the investors deduce $\varphi$ from the knowledge of $\Phi$. Since in addition countries' strategies do not depend on $\hat{\rho}$, which is unknown to them when making the declarations, one has that $\tilde{\rho}=\hat{\rho}+\varphi(1-\hat{\rho})$ and investors learn the true $\hat{\rho}$ from observing $\tilde{\rho}$. Investors wish to maximize their ex-ante return from investment (this will be the characteristic of any equilibrium), hence they will allocate capital $\bar{K}$ so that the marginal return from investment in countries declaring a Good or Bad state of the world is equalized: at equilibrium, the invested capital $K_{i}(g, \tilde{\rho})$ and $K_{i}(b, \tilde{\rho})$ do not depend on the countries $i$ (given that $C_{i} \equiv C$ for all $i$ ) and their common values $K(g, \tilde{\rho})$ and $K(b, \tilde{\rho})$ satisfy $^{20}$

$$
\alpha\left(\frac{\hat{\rho}}{\tilde{\rho}} A(g)+\frac{\varphi(1-\hat{\rho})}{\tilde{\rho}} A(b)\right)(K(g, \tilde{\rho}))^{\alpha-1}=\alpha A(b)(K(b, \tilde{\rho}))^{\alpha-1} ;
$$

Existence of this equilibrium is shown in the Appendix. When $\Phi$ is high enough, all countries always make a truthful declaration about the state of their productivity and $\varphi=0$.

Proposition 6 (stated in the Appendix) guarantees the existence of a one-to-one mapping $\varphi \in$ $(0,1) \mapsto \Phi$ and the following result indicates that this mapping (and its inverse) are decreasing: the weaker the punishment, the more countries lie.

Proposition 1. The fraction $\varphi \in(0,1)$ of liars at equilibrium strictly increases as the punishment $\Phi$ decreases.

In the analyzed equilibrium, investors make their decisions rationally; there is some information transmission between countries and the investors; and some fraction of countries is lying about their state of the world.

We now perform some comparative statics and interpret the implications of the model for the data. We shall use the implications stemming from these propositions to partition countries into different groups that should display different behavior in manipulating (or not) their messages.

Proposition 1 does not handle the case if there were different groups of countries with different levels of punishment (the size of which is common knowledge). Investors would view differently each group of countries, but still they would want to equalize the ex-ante return to capital on both groups. Suppose there are two groups ${ }^{21}$ that vary in the punishment costs of misreporting, that are respectively denoted by $\underline{\Phi}<\bar{\Phi}$.

Proposition 2. Countries in the group with the lower punishment cost $\Phi$ have a higher fraction of liars at equilibrium.

\footnotetext{
${ }^{20}$ We assume that the endowments of capital $C_{i}$ are such that even when being in the Bad state $b$ each country would receive some international flows.

${ }^{21}$ The arguments in Propositions 2-4 can be extended to countably many different groups.
} 
Let $\Theta$ be the cost of abandoning a fixed currency regime for country $i$ if the period capital flows were low after the country truthfully declared $s_{i}=b$. This means, ceteris paribus, given a penalty $\Phi$, that the effective penalty for misreporting for such a country is lower when the true state is $s_{i}=b$. Hence, the above proposition shows that if the countries have the same level of installed capital $C$ a country with a fixed exchange rate would more likely provide false information than a country with a more flexible currency arrangement if they had the same openness to capital flows. Also, if some countries are facing higher punishments because of their previous misreporting or sovereign defaults, Proposition 2 tells us they would be less likely to misrepresent their statistics.

We now show that countries with less installed capital (lower $C_{i}$ ) and therefore relying more on foreign investment will have higher incentives to lie. For the clarity of exposition we again assume that there are only two groups of countries, with respective installed capitals $\underline{C}<\bar{C}$.

Proposition 3. Countries in the group with the lower installed capital $\underline{C}$ have a higher fraction of liars at equilibrium.

When we turn to data, we will interpret the last observations in the following way. Countries with high negative asset positions (i.e., relying strongly on foreign capital), having a need of financing their negative current account and/or a fiscal deficit and at the same time open to capital flows should have higher incentives to misreport their true state of the economy.

In what we assumed so far, capital flows were unrestricted for the mobile international capital. Suppose now that there are barriers to capital flows to some countries, so that only a fraction $\tau<1$ of any investment is obtained ex-post. Then, ceteris paribus, a country with no barriers will have higher incentives to misinform investors in the Bad state of the world as the resulting relative capital flows (when claiming the Good vs. the Bad state) will be more important.

Proposition 4. Countries with lower barriers to capital flows have a higher fraction of liars at equilibrium.

When the economic state of the world (realized $\hat{\rho}$ ) is lower than the expectation of $\rho$, the fraction of liars among all countries is higher.

Proposition 5. The lower the $\hat{\rho}$, the more countries are lying.

This may not be the necessarily true during all "crisis" years when the fraction of countries receiving a Bad state of productivity is high, if such an outcome was expected.

Punishments (possible extensions). We assumed that the punishment $\Phi$ is constant and that the interaction is one shot only. A more complex model could be delivered; for example, investors may punish a country that lied by not investing in it in the future for at least some time. We chose not to model such an interaction, as in repeated games, there are many punishment structures that could sustain different equilibria; which one would be used by investors is an empirical question which was not resolved even in the oft-studied sovereign-debt literature. We also did not do it for parsimony. 
Benabou and Laroque (1992) provide a model with the stage game with similar features to ours (though with players' payoff functions that are easier to analyze) ${ }^{22}$ where many different equilibria with repeated interaction are characterized depending on the initial assumptions.

\section{Data}

For our BOPS data set, the initial date of 1989 is chosen because prior to that year the data are available for few countries and international capital flows were then relatively small; after 1989 many new countries emerge that start providing statistics. We use quarterly data that investors often take into account while making their decisions. Yearly data occurs with a lag and may not be as informative (so may not be actively falsified); moreover we would get four times fewer datapoints. Monthly data are available only for a few countries on a regular basis.

We take all the net figures from the BoP so as to lower the problem of persistence. ${ }^{23}$ We consider this to be an innocuous but a helpful fix. Net values like the current or financial account balances are given by many countries to investors before any gross items are available. Theoretically (see Section 5.1.1), a difference of almost all two exponential sequences (so, net values) will also have a Benford behavior of the first digits. We consider all entries (also Net Errors and Omissions); as the BoP items should sum up to zero, a typical manipulation would require changing at least two series. Net Errors and Omissions is an item that can be inflated to hide unfavorable current account data (Reuters News, 2005). All in all, we have at maximum 13 entries ${ }^{24}$ per country for each quarter, so with 76 quarters of data - at a maximum 988 observations for each country. More detailed statistics for the BoP are not available for a large set of countries and often come with a considerable lag. ${ }^{25}$

We have data for 103 countries for the period 1989-2007 with 69287 observations on the first digits. As the maximum number of observations is then 101764 (103 countries with 13 items reported over 76 quarters), there is a considerable number of missing observations. Many of these missing values appear not to be random, stemming from the fact that countries did not report all BoP entries (especially those that were small) for several consecutive quarters (or at all) at the beginning of the studied period, but started doing so regularly with time. In the first quarter of 1989, we have 517 observations while for the last quarter of 2007 - 1167 of them. An example of an entry that has this pattern is "Financial Derivatives: net" with only 3 countries reporting it in the first quarter of 1989

\footnotetext{
${ }^{22}$ These authors studied a problem of an informed agent which, except for passing her private information to other noninformed traders can trade on this information herself. The game studied there is a game of cheap talk when regarded as a one-stage game, and then a repeated game analysis is provided.

${ }^{23}$ Some items, like Net Errors and Omissions or for many countries Capital Account: net are only reported in net values.

${ }^{24}$ These are: Current Account: net; Goods: net; Services: net; Income: net; Current transfers: net; Capital Account: net; Financial Account: net; Direct Investment: net; Portfolio Investment: net; Other Investment: net; Financial Derivatives: net; Reserve Assets: net; Net Errors and Omissions. We have not investigated any non-net series.

${ }^{25} \mathrm{We}$ do not use the data supplied directly to investors in real time, but what is given to the IMF with some lag. Nevertheless, the IMF is an important lending institution which has an influence on the financial conditions in many, especially non-OECD, countries. The data submitted to the IMF are also used by market participants in their analyzes.
} 
and 45 countries in the last quarter of 2007 . We find 32456 missing observations that are missing in consecutive quarters (so that only 21 of the missing observations occur in 1 quarter only). Among them, we lose at least 4628 observations only due to the fact that 24 countries were created within the sample period so obviously could not report the data prior to their independence (and possibly for some years afterwards). Although some countries report the data for few years or few quarters only with breaks for unknown reasons, we do not consider the missing observations as a major obstacle. Even if some countries may have not reported data for strategic motives (which we cannot outrule) this would work against our method of detecting irregular behavior.

We also use a subset of the data containing only the countries reporting BoP data for at least one quarter and one series a year between 1995 and 2007, keeping only the observations corresponding to the latter period of time. This subset will be referred to as the stable sample while the notion of the full sample will refer to the whole data set. We inspect this sample for the following reasons. The stable sample is a more complete data set with 66 countries between 1995-2007 with 41245 observations out of 44616 all possible values. ${ }^{26}$ Some additional data that we use to provide conditions for slicing the samples (country investment positions, WGI, etc.) are available for this later period. When we scrutinize yearly data only, the number of observations per year remains roughly constant. The countries included therein may also provide higher quality data. For example, Poland is excluded from this sample as it did not report the data between 1996-2000 when it was changing and improving the statistical methodology of BoP reporting. The stable sample is thus our preferred one.

To categorize countries according to their international investment position, we use the updated and extended version of the External Wealth of Nations Mark II database developed by Lane and Milesi-Ferretti (2007). The exchange rate regimes were classified based on information of the IMF in various issues of the "Annual report on exchange arrangements and exchange restrictions" (IMF [1989-2008]) and the data provided by the Fund staff themselves. We opted for the de facto classifications of the IMF. What matters for us is the end-of-period (quarter) status of the regime. Data for fiscal balances was taken from the IMF. Data for institutional quality measures were taken from the World Bank's World Governance Indicators (WGI) database (Kaufmann et al. [2009]) while those for country ratings from the Institutional Investor.

The basic characteristics of the variables in the full and stable samples do not differ substantially (Table II). The major exception is in the exchange rate regimes: a higher fraction of the observations is available for the purely floating regimes in the stable sample than in the full sample (36.3\% vs. $28.2 \%$ respectively) and a lower fraction for fixed exchange rate regimes (37.5\% vs. $45.8 \%$ respectively). The countries in the stable sample have on average slightly higher liabilities but higher net foreign assets and better institutional rankings.

\footnotetext{
${ }^{26}$ Choosing 1994 as the initial year would give us only 61 countries with more incomplete data, while picking 1996 would only raise the number of countries in the sample to 70 . In both cases the alternative choice would lead to fewer observations being included in the stable sample. As we need as many countries and quarters as possible given the arguments advanced in Section 5, the choice of the 1995-2007 sample is a natural one.
} 


\section{The balance of payments data and Benford's Law}

We first recall why the numbers contained in the BoP data should theoretically follow a specific distribution called Benford's Law and then show that this is empirically the case.

\subsection{Benford's Law and its properties for macroeconomic data}

Newcomb (1881) and Benford (1938) independently observed empirically and described a distribution of numbers called since the Benford's Law. The predicted occurrences of the first ${ }^{27}$ digits are given, for all $j \in\{1, \ldots, 9\}$, by $\theta_{j}=\log _{10}(1+1 / j)$; the values of these frequencies $\theta_{j}$ are given in Table I and are plotted in the top left graph of Figure 1.

\begin{tabular}{cccccccccc}
\hline$j$ & 1 & 2 & 3 & 4 & 5 & 6 & 7 & 8 & 9 \\
$\theta_{j}$ & $30.1 \%$ & $17.6 \%$ & $12.5 \%$ & $9.7 \%$ & $7.9 \%$ & $6.7 \%$ & $5.8 \%$ & $5.1 \%$ & $4.6 \%$ \\
\hline
\end{tabular}

Table I: Benford's Law $\left(\theta_{1}, \ldots, \theta_{9}\right)$ for the first digits.

To date, we know of one property and two families of processes that lead the data to be distributed according to Benford's Law. These are processes with exponential growth and variations around them (differences of such processes and/or random exponential growths); taking random samples of random distributions; and scale-invariant (unit-invariant) processes. We label these properties P1, P2, and P3 and this is how we refer to them in the remainder of the text.

We first provide a brief overview of the classes of processes that lead to Benford's Law; mathematical details and further references may be found in Section C.2.1 in Appendix C. The applicability of the Benford's Law to macroeconomic data was already discussed in Nye and Moul (2007) or Gonzalez-Garcia and Pastor (2009), based on the mentioned properties P1-P3 and on the properties of artificially generated sequences.

\subsubsection{P1-Processes with exponential growth}

The simplest generating model is formed by (deterministic) geometric sequences $\left\{a, a^{2}, \ldots, a^{n}\right\}$ with ratio $a$ such that $\log _{10} a$ is irrational ${ }^{28}$; they lead to sets of data that conform more and more closely to Benford's Law as $n$ grows (Diaconis [1977]). Almost all geometric sequences lead to Benford's Law as the set of rational numbers has a null measure within the set of all real numbers. This implies

\footnotetext{
${ }^{27}$ Benford's Law also describes the frequencies of occurrences of the next digits, which we do not use here for the lack of a sufficient number of observations.

${ }^{28}$ Otherwise the sequence of the first digits is cyclic and has asymptotic proportions that are rational and thus are different from the ones of Benford's Law; consider the simple example when $\log _{10} a=2 / 3$, in which case the sequence is $10^{2 k / 3}$ and contains only elements with first digits equal to the first digits of $10^{2 / 3}, 10^{4 / 3}$, and 100 , that is, $4,2,1$; the asymptotic repartition of the first digits is uniform between these three numbers.
} 
that in practice all geometric sequences linked to real data lead the data to obey Benford's Law. This result extends to differences of such geometric processes: for given ratios $a$ and $b$ (such that $\log _{10} a$ is irrational) and any real numbers $\alpha$ and $\beta$, the sets $\left\{\alpha a-\beta b, \alpha a^{2}-\beta b^{2}, \ldots, \alpha a^{n}-\beta b^{n}\right\}$ conform more and more closely to Benford's Law as $n$ grows (Lemma 2.4 in Kaynar et al. [2010]). Finally, all these deterministic results extend to certain sequences of random variables $\left(X_{j}\right)$ and $\left(Y_{j}\right)$, with the respective replacement in the expressions above of $a^{t}$ and $b^{t}$ by $X_{1} X_{2} \ldots X_{t}$ and $Y_{1} Y_{2} \ldots Y_{t}$. The sequences $\left(X_{j}\right)$ and $\left(Y_{j}\right)$ can be formed, for instance, by independent and identically distributed random variables each having a common characteristic function $\varphi$ such that $\varphi(2 \pi k) \neq 1$ for all natural integers $k \neq 0$; this condition is satisfied for almost all distributions. Many other extensions and relaxations of the condition of independence and/or identical distribution exist. ${ }^{29}$

Given this, we may consider macroeconomic statistics that are obtained in some multiplicative way; due to economic, population or price growth, such multiplicative sequences of macroeconomic statistics for a given country should, if observed for a long enough time, result in a collection of numbers with the distribution of first digits abiding Benford's Law. For instance, the series of nominal GDP (exports, FDI flows, etc.) figures can be considered - but not the series of the GDP (exports, FDI flows, etc.) growth rates, since the latter correspond to realizations of random variables $X_{j}$ above. For example, in a stationary economy with constant real GDP (exports, FDI flows, etc.) ${ }^{30}$, this should be the case if long enough time-series are considered when the inflation in each period is, e.g., drawn from a log-normal distribution with a given mean, say 1 , and a constant small variance.

These results extend to net values: the difference of multiplicative sequences of macroeconomic statistics each as exports or imports (the trade balance) would generate Benford behavior of the first digits. In the same way, mismeasurement - for example consistent underreporting of some quantities - will still lead to Benford sequences as long as the nonmeasured part (i.e., illicit trade) can be represented by sequences discussed above. Various extensions can be modeled as special cases of the multiplicative form discussed above, including the case of structural breaks (that could be associated with deep economic crises or large shocks) and/or the presence of errors in data collection. ${ }^{31}$

\subsubsection{P2—Random samples of random distributions}

Hill (1995b) considered the case of data sets with numbers chosen at random according to a twostep procedure choosing first at random a distribution over the positive real numbers, then, drawing

\footnotetext{
${ }^{29}$ See Section C.2.1 in Appendix C for an overview of some recent results.

${ }^{30}$ This will be also true not only in an economy with (zero on nonzero) GDP growth but even with stochastically varying real GDP, as long as the increases or variations can be modeled as the result of successive multiplications by independent and identically distributed random variables.

${ }^{31}$ Suppose that each period a break can occur independently from the past with a constant (small) probability. In this case, the $X_{j}$ are given by a two-step randomization, the first step determining whether a break occurs and the second one providing the adequate multiplicative factor, drawn at random according to a distribution depending on the regime. The same two-step modeling can be applied in the presence of errors in data collection: if the errors can be described in some random multiplicative manner, the obtained sequences will obey Benford's Law.
} 
$k$ numbers according to it, and finally repeating the whole procedure a large number $n$ of times; he provided natural conditions on the distribution of the random probability distribution for Benford's Law to occur when $n$ grows. Janvresse and de la Rue (2004) showed that it suffices to consider random probability distributions taking values in the family of uniform distributions over intervals.

This means that data coming from many different distributions are much more likely to conform to Benford's Law (as observed by Benford himself). Aggregating macroeconomic data of several countries preserves the conformity to Benford's Law — and even should increase it; this is important to remember especially when the number of data points per country is too small (i.e., the series are too short or/and when breaks occur) for property P1 to lead to the conformity to Benford's Law per se. Property P2 works best when the measured quantities differ in scales that may arise naturally for example in terms of the country size. It should work well for our BoP data set, as the scale of the items on the BoP balances clearly is determined by the size of the economy in question. It could lead for example for price levels to converge to Benford's Law, especially if they do not all start at the same initial value (for example, indices with different base years for each country are preferred).

\subsubsection{P3-Scale invariance}

Pinkham (1961) and Hill (1995a) proved that Benford's Law was the unique law such that the distribution of the first digits of numbers drawn at random according to it was stable when the drawn numbers are multiplied by a common multiplicative factor.

This means that the distribution of first digits according to Benford's Law is preserved even if data for a country was provided in home currency and then converted into U.S. dollars (which is the case for the BoP data). The multiplicative factors used to proceed to these conversions are random and change with time; however, since we are interested in quantities that can be written in multiplicative forms, the impact of the conversion into U.S. dollars (i.e., the value of the exchange rate) can be encompassed in the sequence of the $X_{j}$, which should thus describe the multiplicative evolution of the quantity of interest as well as the one of the exchange rate. This way a conversion of a sequence adhering to Benford's Law in one currency to another should lead to a Benford sequence.

\subsubsection{More on the applicability to macroeconomic data}

The most thorough study of conformity to Benford's Law of data in macroeconomics to date is Nye and Moul (2007). They showed through simulations that long enough sequences of nominal GDPs for a set of fictitious countries formed sequences of numbers whose first digits obey Benford's Law, provided that the random economic factors (various growth rates) were set in a proper way. They then illustrated on the Penn World Tables dataset that some subgroups of countries (OECD countries on the one hand, African countries on the other) are such that the data set obtained by aggregating their GDP data conformed to Benford's Law. They also argued that various transformations of nominal data (inflation adjustments, creation of purchasing power indices, etc.) may alter its quality and lead to sets of numbers not conforming anymore to Benford's Law. They used the Benford's Law as a test 
of naturalness of the data, as is the case in other fields, e.g., accounting. They conclude that "broadly speaking, country GDP figures should be consistent with Benford's distribution when countries are heterogeneous in their initial levels (e.g., population, per-capita income, etc.) and then grow."

Gonzalez-Garcia and Pastor (2009) based on the same IFS data set as ours find that in general data conforms to Benford's Law when countries are grouped based on geographical criteria; they in particular consider also the same BoP data set as us with entries transformed into U.S. dollars. They point out that the obtained conformities are hardly related to measures of the quality of the data (as indicated by independent experts) and also explain the atypical behavior of series for some countries by structural breaks. Their tests are however based on annual observations and use fewer data points as we do, thus probably not being able to ensure that $n$ is large enough when calling for property P1. In addition, they do not correct for the persistence issues we mention in the next section. Given the short data span and the frequency, they may not have enough observed cycles.

\subsection{The chi-square test of goodness of fit to Benford's Law}

Such a goodness-of-fit test compares the empirical frequencies $\widehat{\theta}_{j}$ of the digits $j \in\{1, \ldots, 9\}$ of the numbers arising in a given data set to the theoretical frequencies $\theta_{j}$ prescribed by Benford's Law, via the statistic $D^{2}=N \sum_{j=1}^{9}\left(\theta_{j}-\widehat{\theta}_{j}\right)^{2} / \theta_{j}$, where $N$ denotes the total number of first digit observations available. The statistic $D^{2}$ converges in distribution to a chi-square distribution with 8 degrees of freedom as $N \rightarrow \infty$; practitioners think of the distribution of $D^{2}$ to be already close enough to this limit whenever $N \geqslant 30$ and $N \theta_{j} \geqslant 5$ for all $j \in\{1, \ldots, 9\}$ (van der Vaart [1998], p. 243). These conditions are in our case $N \geqslant 5 / \min _{j} \theta_{j}=5 / \theta_{9} \geqslant 110$. This approximation is used for P-values associated with values of the statistic $D^{2}$ in the tables. The above-mentioned test is powerful; it is easy to show by concentration-of-the-measure techniques (see Rivoirard and Stoltz [2009], pp. 57-58) that the probability of not rejecting any distribution of the first digits other than Benford's Law when the first digits indeed conform to Benford's Law is exponentially small in $N$, where the constant in the exponent depends on the discrepancy between the alternative distribution tested and Benford's Law.

The idea of detecting manipulations in the data by tests of conformity to Benford's Law is now well established is auditing and accounting, after the seminal article of Nigrini (1996) and the successful use of his methodology by the authorities of the city of New York, leading to the detection of frauds in seven companies; see Nigrini (1999) for an extended review of many other successful stories. The methodology there is to use as the data set to be tested all quantities appearing in accounts (the values of all individual transactions); the theoretical justification behind this is idea is mainly the property P2 discussed above. Other occurrences and practical uses of Benford's Law can be found, e.g., in Hill (1995b); one can cite, among others, stock market data (Ley, 1996) and census statistics as occurrences and the design of more efficient computers as a possible use. 


\subsection{Global conformity of our proposed data set to Benford's Law}

We first use chi-square tests of goodness of fit to show that our data set indeed conforms globally to Benford's Law, as suggested by the properties P1-P3 and the existing literature. We consider here the full and stable samples, as well as those formed by OECD and non-OECD countries. The first and second columns of Table III indicate the number of countries encompassed by the economic condition considered, as well as the average number of quarters per country. In the third column we report the corresponding $\mathrm{P}-$ value and in the fourth column the value of $N$. Since the $\mathrm{P}$-values are much larger than any conventional threshold for rejecting a null hypothesis, the data set considered globally (and all other subsets here) conforms to Benford's Law.

\subsection{Conformity of typical subsets of the BOPS data set to Benford's Law}

In this paper we consider subsets of the entire BoP data set defined by some economic conditions and show that some of them, for appropriately chosen conditions, lead to subsets whose distributions of the first digits do not conform to Benford's Law. But before doing this we need to show that in the BOPS data set typical (most of the randomly drawn) subsets do obey the latter law!

As we detail below, a persistence issue appears in a significant fraction of the data series, which is compensated by the heterogeneity between countries. Subsets corresponding to a small number of countries and/or containing many consecutive or close-in-time quarters of these countries typically do not conform to Benford's Law while the ones corresponding to the choice of a large number of countries and/or a few number of quarters per country do. This is in line with properties P1 and P2: property P1 may require very large sample sizes, while property P2 indicates that smaller sample sizes for each country can be compensated by grouping the data corresponding to different countries.

\subsubsection{Persistence}

We illustrate persistence on the top part of Table IV, where we estimated the probability that when choosing a given number $C$ of countries at random from our data set, the data subset formed passes the goodness of fit test against Benford's Law at the $5 \%$ level. This table shows that a significant fraction of the subsets formed by $C$ countries, with $C \leqslant 70$, cannot be said to be distributed according to Benford's Law. Only when taking a large number of countries (e.g., $C=80$ ), one gets subsets that typically conform to Benford's Law.

In the subsets grouped by economic conditions, however, fewer countries will be typically involved; fewer quarters too, and this will be the key. Indeed, as is shown in the bottom part of Table IV, the rejection rates become smaller as either the number $C$ of involved countries increases or the number $M$ of quarters picked at random for each country decreases.

This can be explained by persistence of data from one quarter to another and/or structural breaks in the series for individual countries (Section C.2.2 in Appendix C); it is crucial to deal with it when a small number $C$ of countries is involved (otherwise enough heterogeneity is introduced for the 
aggregated data to conform to Benford's Law). We use net items from the IMF BOPS database to reduce persistence as much as possible, but obviously an extra treatment is needed.

One solution is to select only the less persistent series (Section C.2.3 in Appendix C); doing so, however, one may not detect the manipulations on the most crucial series that are watched and analyzed by investors, which can be the most persistent ones as well (e.g., the balance on the current account or goods). Some persistent series in fact may also be such because they are manipulated. This is why we focus on the fact that conformity to Benford's Law is more likely when the data subset is formed randomly by taking few quarters only (relatively to the number of countries) as in Table IV.

\subsubsection{A methodological remedy: Considering random sub-subsets}

We need to quantify whether a large enough proportion of the data subsets conform to Benford's Law for the whole data set to be declared as abiding Benford's Law. This is why we introduce the following new statistical methodology.

To assess the conformity of a subset $D$ of the data, we do not consider all country-quarter pairs of the corresponding subset, but take a small fraction $f$ of them at random and test the conformity of the obtained random subset to Benford's Law (at the $5 \%$ level), a fact that we denote by $R_{D}$, which is therefore a Bernoulli random variable. We repeat this procedure a large number of times, say, 1,000 times, by fixing $D$ but by choosing different sub-subsets of it at random. The average rejection rate will be an indicator of conformity to Benford's Law without the bias due to persistence (and, due to the randomness, to any structural breaks in the data). Formally, the procedure above gives rise to the random variables $R_{D, 1}, R_{D, 2}, \ldots, R_{D, 1000}$, which, conditionally to $D$, are independent and identically distributed according to a Bernoulli distribution with parameter denoted by $q_{D}$. We consider the empirical mean $\bar{R}_{D, 1000}$ of these random variables as the statistic of interest.

The heuristics behind this procedure is to reduce efficiently the average number of consecutive quarters at hand per country, thus weakening persistence, and to consider $q_{D}$ instead of the $\mathrm{P}$-value quantifying the goodness of fit of $D$ against Benford's Law. The repetition of the procedure aims at obtaining a stable result given $D$. Formally, me mean that

$$
\left[\bar{R}_{D, 1000} \pm 1.96 \sqrt{\frac{\bar{R}_{D, 1000}\left(1-\bar{R}_{D, 1000}\right)}{1000}}\right]
$$

is a confidence interval at a $95 \%$ confidence level for $q_{D}$, where, given that the typical realized values of $\bar{R}_{D, 1000}$ are around 0.10 , the precision of the estimation of the rejection rate $q_{D}$ is about $\pm 2 \%$.

To use this new criterion, we first determine the typical values of the statistic $\bar{R}_{D, 1000}$ when $f \in$ $\{5 \%, 10 \%, 20 \%\}$ and $D$ is a set drawn at random $^{32}$ from Benford's distribution with a given size

\footnotetext{
${ }^{32}$ Of course, it is immediate that in this case, the expectation of $\bar{R}_{D, 1000}$ (with respect to the choice of $D$ and the random subset) is $5 \%$ but we want a sharper idea of its distribution, namely, good estimates of its tail.
} 
$N$. Results are in Table $\mathrm{V}$, which provides estimates of the quantiles of the underlying distribution according for $f=10 \%$, which is the values mostly used in this paper; the quantiles for $f=5 \%$ and $f=20 \%$ can be found in Table XIV in Appendix C. These estimates were constructed by running 1,000 times the above procedure on randomly generated sets $D$. This involved computing $10^{6}$ goodness-of-fit tests per cell; because of the computational cost, no sharper estimates based on more repetitions are given.

This method provides us with another test of conformity to Benford's Law of subsets $D$; this test, given $D$, compares the obtained value of $\bar{R}_{D, 1000}$ to the above quantiles and is then able to associate a P-value with the hypothesis of conformity to Benford's Law. This is done by identifying in which interval of the last line of Table $\mathrm{V}$ (corresponding to the chosen $f$ ) this value lies in. For instance, if the realized value of the statistic $\bar{R}_{D, 1000}$ is 8.5 and $f$ was chosen equal to $10 \%$, the corresponding $\mathrm{P}$-value is between $1 \%$ and $5 \%$; or if the realized value is 5.4 and $f$ was chosen equal to $5 \%$, the the corresponding $\mathrm{P}-$ value is larger than $5 \%$.

The subsamplings in proportions $f \in\{5 \%, 10 \%, 20 \%\}$. are called, respectively, 1-in-20 (when $f=5 \%$ ), 1 -in-10 (when $f=10 \%$ ), and 1-in-5 (when $f=20 \%$ ) criteria. We include the 1-in-10 criterion in our main results as this already delivers good randomness of the sampled data, but is not as demanding in terms of the required observations as the 1 -in-20 criterion. ${ }^{33}$

\subsection{Our new methodology and the conformity of the BOPS data set}

We include all the criteria discussed above (as well as the one using less persistent series described in Appendix C) in Table III and find a good adequation of the first digits of the BoP data to Benford's Law confirming our findings from Section 5.3. This is true for the full and stable samples. It does not appear either important whether we restrict the samples to include only OECD members (as of 1989 ) or not. This is very important, as we do not find that non-OECD countries have non-Benford distributions of the first digits as an entire group taken together, which is different from the results of Nye and Moul (2007) on the Penn World Table dataset; we further investigate groups of countries that may have driven the results of Nye and Moul (2007) in Sections 6.5 and 6.6.

\section{Empirical results}

We discuss here in detail the results for our preferred "stable" subsample of countries which provided data at least once each year in the period 1995-2007 and back them with the results from the full sample and other robustness checks. We perform traditional chi-square goodness-of-fit tests and our developed 1-in-10 randomized tests on the subsamples grouping country-quarter pairs by economically meaningful characteristics to detect any irregularities in the first-digit behavior. The null

\footnotetext{
${ }^{33}$ To ensure that for each chi-square goodness-of-fit that need to be performed at least 110 observations, as mentioned above, are available, at least 1,100 and 2,200 are respectively needed to compute the 1-in-10 and 1-in-20 criteria.
} 
hypothesis is that the first digits of the data are drawn from Benford's distribution. We consider that the null is rejected if it is so for both tests. Based on our model in Section 3, we believe that some groups of countries have higher incentives to tweak their BoP statistics than others. Fixed exchange rate regime countries may be wary to provide truthful information about the developments in the BoP because of a fear of an attack on their currency that unfavorable information may trigger. Countries with high net foreign asset positions in terms of GDP, those having negative current account balances or fiscal deficits may fear that too much of negative information in the BoP data may spur a nervous reaction of investors and cause capital outflows, which they would like to avoid.

In grouping countries we investigate subsets that are the unions of various conditions. While investigating the specificity of countries partitioned according to their exchange rate regimes, current account or fiscal balances, we also look at subgroups of countries that have relatively lower net foreign assets excluding foreign direct investment to GDP (in tables, denoted as NFA_EXCL_FDI_GDP) or higher foreign equity liabilities to GDP ratio (EQ_LIAB_GDP). Additional criteria (for example for the exchange rates) are presented in tables in Appendix C. For the international investment positions, we took the top $75 \%$ of countries ranked in terms of negative net foreign assets or foreign liabilities. ${ }^{34}$ In all cases, we use previous year's figures for grouping countries. So, e.g., to test for a group of countries with fixed exchange rate regimes that also have low net foreign assets (excluding FDI) in the stable sample, we took all the countries which had the net foreign assets (excluding FDI) to GDP ratio lower than -0.0201 in the preceding year that also had a fixed regime. ${ }^{35}$ As a measure of capital openness we used the foreign equity liabilities/GDP ratio. A relatively higher value of this indicator shows that a country is de facto more open to private capital flows than others, and hence may be also more prone to capital outflows. ${ }^{36}$

The amount of data that we were able to gather allows for grouping countries into many different subsamples; however with many restricting conditions these subsamples become small, containing few countries with many quarters of data which would lead to easy rejections of Benford's distribution due solely to persistence, as discussed in Section 5.4. In such cases we want to rely on the 1-in-10 condition but then we need at least 1,100 observations in the sample, as discussed in Section 5.4.2. Hence we are unable to slice the data very finely; this is against our story as we cannot condition very well in each category for countries that may have higher incentives to cheat.

\footnotetext{
${ }^{34}$ In the robustness checks, we also use the top $90 \%, 80 \%, 66.66 \%$ or $50 \%$ of countries (not reported).

${ }^{35}$ The quantiles of a given economic quantity (e.g., net foreign assets to GDP) were computed by considering all the available values of the quantity as countries and quarters vary; that is, at most one value of the quantity per countryquarter pair of the sample (full or stable) was considered: none when the quantity was unavailable and one when the data contained information about it. For this reason the number of available observations in, for instance, the top $10 \%$ or lower $10 \%$ of the country-quarter pairs for this criterion may differ; the difference is due to the grouping according to the criterion by itself.

${ }^{36}$ We did not use the total portfolio liabilities (equity and debt) as many debt liabilities for a country consist of foreign denominated debt which is traded outside of the country borders. There are also fewer data points for this measure.
} 
Robustness checks for our findings. We offer several robustness checks of our main goodnessof-fit tests, most of which are shown in Appendix C. First of all, we typically test whether for the complement of the set for a given restricting condition (or an alternative regime in the case of an exchange rate regime) we reject Benford's distribution for the first digits as well or not. Such a rejection would make our claim of the relevance of a potential category for grouping countries and detecting data manipulation vacuous. Secondly, we seek a confirmation of our results for the stable sample in the full sample as well. Next, we want to know whether it is not only one country which drives the result for a particular category. This may indicate that out of the whole group only one country is either providing false information or, for example, suffers from a dire problem of persistence in its quarterly data. The fact that such a country would drive the results would invalidate the generality of the claim. This number of countries driving the results (which we label as the "stability index") is quantified by the number of countries which, when excluded from the subset, lead to the acceptance of the null hypothesis at a level of $10 \%$; that is, we count the number of countries such that when they are excluded one at a time the P-value associated with the goodness-of-fit test to Benford's Law increases from less than $5 \%$ to more than $10 \%$. We would like our stability index to be zero when we expect to reject the null hypothesis. We also test for the rejection of Benford's distribution for the less persistent items and offer results for different criteria based on random sub-subsets (1-in-5 and 1-in20). Finally, in Section 6.6 we rerun the tests for our principal results excluding countries with poor governance to assure that the worst countries in terms of institutional rankings are not responsible for our findings.

\subsection{Different exchange rate regimes}

In Table VI (see also Tables XVIII-XXI in Appendix C) we present the tests for the conformity of the first digits of the BoP data with Benford's distribution for country-quarter pairs groups in terms of their exchange regimes at the end of a quarter. The left-hand column presents the characteristics of the groups considered. Additional groupings are presented in Tables IX and X.

Any exchange rate regime where the intervention of the central bank was substantial (a currency board, a conventional peg, a crawling peg, a crawling band) was labeled in our data set as "fixed."37 Such a wide range of regimes was classified together to have a large number of different countries. We label a regime as "floating" when it was deemed by the IMF as being independently floating. ${ }^{38}$ It is to note that the currencies that were a part of the European Exchange Rate Mechanism I or II in a

\footnotetext{
${ }^{37}$ Consequently, for example crawling band regimes prior to 1998 , when the IMF adopted a new classification, were coded as being fixed from the category of managed floating.

${ }^{38}$ The remainder of the countries have regimes which are difficult to classify into either category. For some managed exchange rate regimes ("Managed floating with no predetermined path for the exchange rate" in the IMF nomenclature) it is difficult to make a call whether it is a floating regime or not; and we preferred only to make conservative assessments. Secondly, we classify here also countries when they do not have any own legal tender (Panama, El Salvador or Ecuador). Indeed, in our tests, countries with regimes that are neither fixed or floating exhibit different behavior than the two "ideal" regimes (see Section 6.1.1).
} 
given quarter are considered by the IMF to belong to the fixed category prior to the introduction of the Euro and as floating afterwards.

First of all, we observe that the null hypothesis of conformity to Benford's Law is rejected for fixed exchange rate countries both for the stable and full samples at a $1 \%$ significance level. The subsets of countries that have fixed exchange rate regimes contain many countries (49 countries with 26.6 quarters on average for the stable sample and 84 countries with 32.6 quarters on average for the full sample) so that we believe based on Section 5.4.1 that the neither persistence nor structural breaks are driving our results. The 1-in-10 criterion confirms this. The important thing to notice is that any irregular behavior detected here may not be country specific, as many countries in our samples (27 and 40 respectively for the stable and full samples) change the fixed exchange rate regime to floating (or the other way round) at least once.

Moreover, after imposing more restrictive conditions groups of countries with fixed exchange rate regimes have distributions of the first digits for which the hypothesis of Benford's distribution is rejected at a $1 \%$ level for all series. The 1-in-10 criterion confirms our findings as these statistics are always greater than 10. More precisely, the level of significance of the rejection increases considerably when we further refine the picture and consider countries which belong to the upper $75 \%$ in terms of the (negative) net foreign assets (with or without foreign direct investment), equity liabilities, and the size of their total foreign liabilities (shown in Appendix C). This is also true for fixed exchange rate regime countries that have negative current account or fiscal balances (Tables IX and X). This supports our hypothesis that countries with fixed exchange rate regimes that would be more sensitive to capital flows have an interest in tinkering with the signals that they send out to investors. In particular, the rejection rate of Benford's distribution for countries with fixed exchange rate regimes and relatively liquid equity markets within our sample (which means they need to be de facto open to individual financial flows) is $0.00004 \%$ in the stable sample and $0.0007 \%$ in the full sample! This occurs for a large number of countries (38 and 58 countries respectively) in this category with an average data span of 17.8 and 24.2 quarters. Our findings square with the model that we presented in Section 3: that the countries with fixed exchange rate regimes may wish to misreport data; and among these countries it is those that have higher negative net asset positions or higher openness to capital flows that have more incentives to do so.

These results survive our stress tests (shown in Tables XX-XXI in Appendix C). For the stable and full samples, the hypotheses of Benford's distribution of the first digits are still rejected even when we exclude from the sample each country one-by-one (as the values "0" for the stability indexes indicate); which means that there may be several countries in a given group that are responsible for the result. Even when we run the tests on less persistent series of the BoP, we still get rejections of the hypothesis of the first-digit distribution being Benford at a $5 \%$ level-except for the full sample for the fixed exchange rate regimes that have more liquid equity markets or the entire unconditioned group. The 1-in-5 and 1-in-20 criteria tell the same story as the 1-in-10 criterion. These provide a strong indication that the rejections may not be due to the persistence of the data. We also run the tests excluding the entries on the reserve assets, which in fixed exchange rate regimes may experience 
large movements due to the readiness of the central bank to buy and sell currency at a prespecified rate (column 4 in Tables XX-XXI in Appendix C). This is important, as this is the only item in the BoP that a central bank can control directly. It does not seem thus that the unusual distribution of the first digits for fixed regimes comes from the activity in this entry and hence directly from central bank intervention. Taking different quantiles for the conditions preserves all of the results for the top 90, 80, and 66.66 percents of the conditions and most for the 50 percent (however, we start having few observations here for some conditions). The picture does not change when we condition on contemporaneous year values of net foreign asset/GDP or liabilities/GDP ratios.

Our results for fixed exchange rate regimes contrast strongly with similar tests for the floating exchange rates regimes, for which the null hypothesis cannot be rejected even for one category, also when we use the same conditions on the investment positions of a country (Tables VI, IX and X). Notice also that the number of countries with floating regimes included in each sample is always lower than for the fixed regimes in the same category; again this may be an indication that the persistence issue does not matter here and that properties P1-P3 are at work here.

It seems odd that our results would occur only due to the specificity of the economic processes under a fixed exchange rate regime. The fact that some items of the BoP like changes in reserves may be more variable in a fixed exchange rate regime should not matter (and indeed, when we exclude the reserves entry, the conclusions are not different - cf. above) as long as the processes generating the data are well behaved and conditions P1-P3 are preserved. In a fixed exchange regime, there is still inflation, economic and population growth that would lead the BoP data to evolve according to a process that leads to Benford's Law; and we still aggregate across many countries. Some less vulnerable fixed exchange regimes country groups do exhibit first digit distributions for which the hypothesis that these are drawn from Benford's distribution cannot be rejected - for example for countries with positive fiscal balances (Table X), OECD countries as of 1989 (line 5 of Tables XVIII-XIX in Appendix C), countries with relatively low liabilities/GDP ratios in the sample (not shown), or those with Institutional Investor ratings ${ }^{39}$ above 68 (not shown). Also, the rejections of the null hypothesis for vulnerable countries with fixed exchange rate regimes (those more indebted or being more open to capital flows) are stronger than for fixed regimes that are not vulnerable (if the null for the latter is rejected at all). Another question thus appears: can this be driven by less developed countries that for example score badly in terms of the quality of institutions? ${ }^{40}$ This again seems unlikely, as we obtain increases in the strength of rejections (lower P-values, higher 1-in-10 criteria) when adding further conditions. In particular, we obtain strongest rejections for countries with fixed regimes and most internationally open capital markets, which is typically the feature of more developed countries (see also Section 6.6 for further discussion and tests of this issue).

It thus appears that among countries with fixed exchange rate regimes there might be some (but

\footnotetext{
${ }^{39}$ According to Reinhart and Rogoff (2009) such countries are advanced economies that are credible debtors.

${ }^{40}$ When we split the countries at the median of their WGI scores, it turns out that fixed exchange rate regimes are adopted equally often by both groups created in this way. See Table XLVII in Appendix C.
} 
probably not all!) which strategically misinform investors as the distribution of the first digits of the BoP data they report is unusual. This is true especially for countries which may face higher outflows due to the stock of liabilities they owe to the outside world. It becomes even more so for countries which are de facto open in terms of capital flows (and have higher equity/GDP liabilities than other countries). There is economic rationale why this may be the case: in a fixed exchange rate regime the aggregate information contained in the behavior of the exchange rate is missing, and investors need to rely more on the information provided by a country, which may want to tweak it in order to put itself in a more favorable light.

\subsubsection{Subcategories of exchange rates}

We report here further results of our tests among finer IMF exchange rate categories. These tests are less reliable, as we have fewer countries in each group, and persistence may be a larger problem. We present two studies in Table VII (supplementary tables in Appendix C). First, we compare different exchange rate regimes. In the top panel of Table VII we observe that the Benford's Law is rejected for the first digits of data in both Chi-square and 1-in-10 tests from countries with crawling peg regimes. Using our 1-in-10 criterion we also find rejections at a 5\% level for countries with currency board and managed float regimes. This, however, may not be the best metric to measure countries with vulnerability of their exchange rate regime. For example, Cape Verde, a country with a conventional peg regime throughout our sample, has very little capital flows as measured by equity/GDP held by foreigners (a maximum of $0.0055 \%$ in the sample). Therefore, we additionally scrutinize countries that are de facto more open to capital flows (lower panel of Table VII), and therefore may be more vulnerable to any currency attacks or rapid capital movements. Interestingly, now we reject the Benford distribution for the first digits for all fixed exchange rate types and managed float regimes as well when using $\mathrm{P}$-values from Chi-square tests and all except conventional pegs using the 1-in-10 criterion. ${ }^{41}$ This means that we get rejections for the countries which manage their currencies and are vulnerable to capital flows. This is also true for countries with currency board regimes - and these are the same four countries providing data as in the stable sample - but for different (shorter) time periods when they experience higher capital flows. On the full sample, for all fixed exchange rate regimes with higher capital flows the null can be rejected at a $5 \%$ level both in Chi-square tests and the tests based on the 1 -in-10 criterion.

\subsection{Countries with high negative foreign asset liabilities}

In Table VIII (see also Tables XXVI-XXIX in Appendix C) we show the P-values of tests for different centiles of the data when countries are ranked according to the ratios of net foreign assets excluding

\footnotetext{
${ }^{41}$ For the currency board regimes, due to a lack of a sufficient number of observations we could not calculate the 1 -in-10 criterion. The 1-in-20 criterion that could be calculated strongly points to a rejection at a $1 \%$ level.
} 
foreign direct investment (FDI) stocks to GDP for the stable sample. This is the most apt measure of the capital stock that could rapidly move out of a country; FDI flows, which are largely immobile in the short run, are excluded, and we take the net value of assets. We use the previous year's value for the current year quarters to group countries.

For the first digits of the BoP data for the $10 \%$ and $25 \%$ of countries with the worst net foreign asset position (excluding FDI) in the stable sample the null hypothesis is rejected at the $5 \%$ significance level, with the 1-in-10 criterion confirming this (for the top $10 \%$ countries on the full sample). These results survive several robustness checks (shown in Tables XXVIII-XXIX in Appendix C). First, for the complement of the sets defined by conditions we cannot reject the null hypothesis of conformity to Benford's Law. With the exclusion of one or more countries we still reject Benford's distribution for the previously flagged categories as indicated by the stability indexes. In addition, we obtain a rejection of Benford's distribution for the first digits of the data for the $10 \%$ most indebted countries on the stable sample also for the less persistent series. The 1-in-20 and 1-in-5 indicators give the same indication as the 1-in-10 criterion.

We conclude that countries that have a high ratio of liquid indebtedness/GDP provided, in contrast to others, BoP data which had an unusual distribution.

We do not get consistent and strong results in terms of the observed patterns for country groups created using other measures of investment positions. In particular, for the full sample we obtain rejections of Benford's distribution of the first digits also for countries with the highest net foreign asset positions and the lowest liabilities/GDP, although we get similar results as for countries with net foreign assets (excluding FDI) for the stable sample. These series may be less pertinent for our tests. For example, the fact that a country has a high total liability/GDP ratio, like Switzerland, may not per se be an indicator that it is vulnerable if it is has high assets as well. It may also mean that we cannot condition the data well enough, for example we do not control for many features (like differences in technology) which may affect the payoffs from misreporting. The data on liabilities may not be free of errors as well; and these are data that we have on a yearly basis only. Therefore, if the data set heterogeneity is large, the picture may be blurred with these less precise measures.

\subsection{Countries with negative current account balances}

We report in Table IX (see also Tables XXX-XXXIII in Appendix C) the results of tests for countries with negative current account positions in a given quarter (labeled as $\mathrm{CA}$ in the tables).

By taking into account the $\mathrm{P}$-values and the 1-in-10 criterion we observe that in both the the stable (P-value of $0.2 \%$ and 1 -in-10 criterion at a $1 \%$ significance level) and the full sample (P-value of $5.6 \%$ and 1-in-10 criterion indicating rejection at less than a $2 \%$ significance level) we obtain a rejection of the conformity of the first digits of the BoP data to Benford's distribution for countries that have negative current account positions in a given quarter. 63 out of 66 countries at some point in time have a negative current account figure (99 out of 103 for the full sample); this shows that the rejection of this condition is not due to some country-specific data dissemination practices but rather 
to what is reported when the country in question is borrowing from the rest of the world in a quarter. The same is true when we additionally select countries with the highest level of net indebtedness (with or without FDI), fixed exchange rates or fiscal deficits (shown in Table X) that are running negative current accounts.

The robustness tests are presented in Tables XXXII-XXXIII in Appendix C. In general they confirm our findings with some caveats. For the main condition - negative current account position in a quarter - after excluding countries one-by-one from the sample we still always reject Benford's distribution for the first digits of the data for the stable sample. It seems that some additional conditioning (for example, choosing countries with high liabilities/GDP ratios, with or without FDI accounted for) have in fact little importance in characterizing countries with unusual first-digit distributions, as we reject the null hypothesis for the complement of the set. The 1-in-5 and 1-in-20 criteria confirm the findings of the 1-in-10 criterion.

Overall, the rejections we find seem to indicate that countries requiring the financing of their borrowing (as their current account balances are negative) may be willing to misinform investors, especially among the countries included in the stable sample.

\subsection{Countries with fiscal deficits}

We group countries also according to whether they had a positive general government fiscal balance in a given year or not (labeled as FISC in the tables). Results are shown in Table X in the main text as well as Tables XXXIV-XXXVII in Appendix C).

The evidence here is weaker than in the case of countries grouped according to other economic criteria. Global P-values for country groups with either a positive or negative fiscal balance are below $5 \%$. For the countries with positive balances this result is however not confirmed for the full sample, and for the stable sample the result is driven by 2 countries, exclusion of either of which removes the rejection. Because the Benford's distribution is rejected for the complement of the sample as well, we do not conclude that a negative fiscal balance by a country itself is consistent with any strategic data manipulation. However, further investigation shows interesting patterns.

When we study the grouping of countries according to a fixed or floating exchange rate regime, we find a rejection of the Benford distribution of the first digits for the BoP data for countries with a negative fiscal balance and fixed exchange rates (a P-value of $0.02 \%$, corroborated on the full sample and by our 1-in-10 criterion). In contrast, countries with a fixed exchange rate regime but reporting a budget surplus display a very good adequation of their statistics to Benford's Law (a P-value of $70.6 \%$ ). We do not reject the Benford's Law for the first digits of data from countries with floating exchange rates either.

When we condition countries according to deficits or surpluses in their current account and fiscal balances (therefore creating four different groups), we reject the Benford's Law only for the group 
of countries with both a negative current account and a fiscal deficit ( $\mathrm{P}$-value of $0.007 \%) .{ }^{42}$ This group contains the most countries and observations among the four possible groups according to this partitioning in the sample. The finding is confirmed for the full sample and by our 1-in-5, 1-in10 and 1-in-20 conditions. Countries with twin deficits - in the current account and with negative fiscal balances - would be the most vulnerable among all the four possible groupings, ceteris paribus: they would be in need of borrowing to cover the fiscal deficit, and doing so (on the macroeconomic aggregate level) from international investors.

These results for the two aforementioned groupings survive our robustness checks. The "stability" index is equal to zero meaning that no one country is driving the rejections. 1-in-5 and 1-in-20 criteria give the same indications as the 1-in-10 criterion. The results are corroborated on the full sample.

Although for either the global partition or among the $75 \%$ of countries with the lowest in-sample NFA (without FDI) we get consistent rejections for both groups of countries (with either positive or negative fiscal balances), we find two highly interesting patterns nevertheless: countries with negative fiscal balances and fixed exchange rate regimes or current account deficits display unnatural patterns in their first-digit data.

\subsection{Quality of institutions and data provision}

We also study how institutional quality affects the behavior of the firsts digits of provided data. For this purpose, we use various WGI from the World Bank and report the results in Table XI (main results for all indicators, on the stable sample). Detailed tables for all WGI indicators along with robustness checks are omitted from the main text but are available in Appendix C.

Both the $\mathrm{P}$-values from the goodness of fit tests and the 1-in-10 criterion indicate that countries ranked below the 50th percentile (and below the 25th as well) in the WGI data set in terms of corruption, political stability and the rule of law and below the 25 th percentile in terms of government effectiveness (in terms of the global WGI data set) exhibit BoP data with first digits that have nonBenford behavior. It appears thus that countries with poor institutions provide data of low quality. This is a finding similar to Nye and Moul (2007) who show that in samples of economic data from the Penn World Tables for non-OECD countries the hypothesis of Benford's distribution of the first digits is rejected (but remember that for our BOPS data this is not the case for the entire group of non-OECD countries, as witnessed by Table III). For rankings in terms of voice and accountability and regulatory quality we obtained no consistent and interesting results.

For all the categories flagged above the rejection of Benford's distribution for the complement of the set (i.e., for countries with better institutional rankings) of the first digits fails. The exclusion of one of the countries from the samples does not drive the results mentioned above, except for the

\footnotetext{
${ }^{42}$ For the group with positive fiscal and current account balances, the 1-in-10 criterion based on studying randomly selected subsets of the data points to a rejection at a $5 \%$ level with the similar pattern for the full sample; but this is not confirmed by the $\mathrm{P}-$ value, so we do not consider it as a category for which we have a rejection.
} 
lower 25th percentile of government effectiveness in the stable sample. For tests ran on less persistent series, we can reject Benford's distribution at the $10 \%$ significance level for the countries ranking lowest $25 \%$ in terms of government effectiveness and political stability for both samples, and for those ranking most corrupt for the stable sample; the evidence based on these is weak. The 1-in-20 and 1-in-5 criteria give rejections for the same categories, albeit sometimes at different conventionally accepted significance levels, as the 1 -in-10 criterion.

Our results lead us to believe that countries with poor institutions produce BoP data with irregular first digit behavior. However, we do not have a story why such countries would manipulate the data. The answer may be that it is not due to bad data collection procedures or methodologies. ${ }^{43}$ Firstly, even high measurement errors should in practice cause the data to have first digits obeying Benford's Law if the errors are well behaved, in view of the property P1 (see Section 5.1.1, as well as the example described in the next section). Secondly, when we look at the tests based on the less persistent series, there is hardly any evidence that countries with bad institutions do have weird first-digit distributions. If the rejection of Benford's distribution would be caused by bad data collection, the first-digit distribution of the less persistent series should be also affected. Thus, a simple explanation for the rejections for these groups of countries may be that their data exhibits persistence. Another story may be that the institutions in these countries have lower scruples or fewer control mechanisms (so that rightly they are ranked as having bad institutional quality) that prevent data falsification. A different point is that such countries may find themselves more likely in a situation that encourages misreporting. For example, as witnessed by Table XLVII in Appendix C, countries with poor institutions in our sample typically are more indebted to foreigners, adopt floating regimes less often, and are more likely to have negative current account and fiscal balances.

\subsection{Are poor countries with bad statistical agencies driving our main results?}

A valid question in this context is whether our main findings in Sections 6.1-6.4 are driven by bad data collection procedures employed in some countries or not. Moreover, Young (2009) claims that many entries for data in the UN or World Bank data sets, for example for Sub-Saharan countries for growth or price level data, are not based on actual measurements by governments but on extrapolations or from other sources. Hence, the data that we might be working with could be either subject to errors during collection or be actually ad hoc or artificially created by various agencies.

We are not aware of how grave the problem of mismeasurement of BoP entries is for the countries included in our sample ${ }^{44}$ and are not aware of the IMF extrapolating the BoP data that are obtained from monetary and statistical authorities of countries. We note that the data we use are available for only 103 countries for the studied period 1989-2007 and for many countries (and data series) there

\footnotetext{
${ }^{43}$ Gonzalez-Garcia and Pastor (2009) compare the quality rankings of data collection methods of various countries with the rejections of the Benford's distributions of their macroeconomic series, finding no consistent pattern between the two.

${ }^{44}$ Though Curcuru et al. (2009) argue the mismeasurement of the BoP entries even for the United States is severe.
} 
are gaps. We have 14 Sub-Saharan countries (including South Africa) in the full sample while only 4 (including South Africa) in the stable sample. If we look at all WGI indicators characterizing the countries in the data, we have countries which boast on average governance scores that are higher than the means for the entire WGI data sets (which contains for 2007 up to 215 entries). For example (as witnessed by Table II), for the most relevant WGI indicator in this respect - government effectiveness - the average rank value for the full sample is 60 while for the stable sample 63.5 (whereas the average rank value in the WGI data set by construction is 50).

Theoretically, errors in measurement should not matter for our results as long as they can be represented by an appropriate stochastic process (see Section 5.1.1). For example, if the goods balance is increasing exponentially but its multiplicative variations are measured with independent errors which are identically distributed ${ }^{45}$ (possibly according to a distribution which is centered around 1 , or according to positively or negatively skewed distributions with respect to 1 which could account for repeated over- or under-estimation of some quantities), the series should adhere to Benford's Law if enough datapoints are observed.

In order to address the issue empirically, we reran our basic results excluding Sub-Saharan countries or those with bad governance scores. We present the results for the most relevant categories in Table XII for the stable sample (see also Tables XLVIII-LI in Appendix C). We see that our basic result on the behavior of the first digits of the BoP statistics for fixed versus floating regimes does not change, and the strength of the rejection of the null hypothesis for the fixed regimes is similar both when we exclude only Sub-Saharan countries (keeping South Africa) on the basis that these countries might not have developed capabilities for clean data collection, or when we exclude countries ranked in the lowest $25 \%$ in terms of government effectiveness in the WGI data. If we look at some additional conditioning, considering for example the exchange rate regime and the openness to equity flows, again the picture is the same. In this case, we are not surprised: very few countries with bad governance have high equity liabilities and also fixed exchange rates (the average government effectiveness rank for countries with a fixed exchange rate in the stable sample and equity liabilities/GDP $>0.0098$ is 70.8). The results are confirmed for the stability index for these results (0) as well as if we only look at the less persistent series - again we obtain a rejection for the fixed exchange rate regimes but not for floating ones. If we exclude Sub-Saharan countries from the group of countries that have the lowest net foreign assets to GDP ratio (excluding foreign direct investment liabilities), the rejection patterns are confirmed. The evidence is weaker when we exclude from the same group the countries with lowest government effectiveness but we still obtain a rejection at $5 \%$ when we scrutinize the $\mathrm{P}$-value. For the countries with a negative current account in a given quarter, the null hypothesis is rejected at $0.1 \%$ level when we exclude Sub-Saharan countries and at $6.7 \%$ when we exclude those with lowest government effectiveness scores. The results for countries grouped by fiscal balances are the only ones for which we cannot obtain rejections after countries with poor institutional scores are

\footnotetext{
${ }^{45}$ Section C.2.1 in Appendix C indicates however that these assumptions of independence and/or identical distribution can be strongly relaxed; more general random errors can thus be considered.
} 
dropped from the sample. Nevertheless, for the groupings of interest for which we claimed rejections in Section 6.4 - countries with negative fiscal balances and fixed exchange rates or countries with twin deficits - we still reject the Benford distribution of the first digits of the data when we exclude countries either ranked in the lowest $25 \%$ in terms of government effectiveness or the Sub-Saharan countries (except South Africa) for the stable sample at least a $5 \%$ level (results not shown).

Based on our discussion above, we do not believe that our results are driven by poor countries with bad data collection procedures.

\subsection{Other conditions for defining the subsets of the data}

We have tried various other conditioning of the data in our search of unusual reporting patterns. ${ }^{46}$

First of all, we studied (akin to Section 6.2) whether countries with more capital openness (defined as high foreign equity liabilities/GDP ratios) exhibit non-Benford distribution of their first digits of the BoP data. Scrutinizing the tails of data sorted according to this measure, we find no results that this is the case.

Next, for countries that joined the Euro zone we inspected the period in which they had to maintain a fixed exchange rate regime and satisfy certain economic performance criteria for admission. We do not find any evidence that the BoP data are unusual for up to 5 years prior to joining the zone. However, interestingly, we find that the Benford distribution is rejected for the first digits of the BoP statistics for these countries after joining the Euro zone. For the full sample, the P-value is $0.08 \%$ and the 1-in-10 criterion 14.6 (both statistically significant at 1\% level) with 4623 observations and 11 countries (Belgium and Luxembourg are not in our data set), while for the stable sample the P-value is $0.18 \%$ with a 1 -in-10 criterion of 14.1 (4259 observations and 10 countries).

We also investigated countries with episodes of sovereign default and banking crises (data on their timing obtained from Reinhart and Rogoff [2009]). For countries that defaulted on their sovereign debt within our sample period, we do not find rejections of the null hypothesis either for one, two or three year brackets preceding a default (results shown in Table LIII in Appendix C). The first digits of the statistics released by countries three, five, ten and twenty years after default appear to conform with the Benford distribution. This is consistent with investors threatening a more severe punishment for future default (or misinformation) but also with lower capital flows after a default occurs. After default, a country's actions may be also scrutinized more closely by investors or the IMF. However, we find evidence that within one year after enduring banking crises countries report suspicious distributions of the BoP data. For these, we can reject the hypothesis that the first digits

\footnotetext{
${ }^{46}$ For geographically-based country groupings (see Section C.4.2 in Appendix C), we rely on the 1-in-10 criterion as the persistence issue may be severe. We find that countries in Africa and the Middle East (for both the full and stable samples), in Latin America and Eastern Europe (for the stable sample) have suspect distributions of the first digits. This should be taken with caution as there are relatively few countries in each group with many quarters of data each, which may make rejecting the null hypothesis easier (tests on the least persistent series do not confirm any of the findings). There may be also too little heterogeneity for property $\mathrm{P} 2$ to be able to compensate for the possible persistence issues.
} 
conform to Benford's distribution at $0.2 \%$ with 5,094 observations on the full sample and $5.6 \%$ for the stable sample $(2,487$ observations $)$.

There seems not to be any evidence that countries suffering substantial falls in their Institutional Investor country credit ratings, either contemporaneous, lead or lagged, offer suspect statistics.

We found no strong results for countries grouped by the level of their year-to-year inflation, though our 1-in-10 criteria reject the Benford distribution for the first digits of statistics from countries with an inflation rate higher than $10 \%$ both for the stable and full samples at a $2 \%$ level.

We inspect our data for main categories on a year-by-year basis in Section C.4.1 in Appendix C. We find that rejections of the Benford distribution for many vulnerable country groupings for 1996, 1997 and 2006. For 1997, one can rationalize the finding in favor of Proposition 5: a global, unexpected crisis put many countries in a position where they misreported their data. We do not know, however, what were investors' expectations in 1997 (and for the other years as well), so we do not draw any conclusions from this exercise.

\subsection{Summary of the empirical results}

We find results consistent with the hypothesis that countries strategically provide manipulated data to economic agents. We observe rejections of Benford's distribution for the first digits of data issued by groups of countries that are more vulnerable to high capital outflows. From the main results shown in Tables VI, VIII, IX and X, we find such pattern in 10 out of 13 groupings with the three inconclusive ones involving partitions using fiscal balances. These rejections are rather category than country specific, as data from many countries for different quarters enters different categories. We obtain the strongest (also most robust) results for countries with fixed exchange rate regimes though we also find that countries with highest levels of net indebtedness and those that were running current account deficits have first digits that have unusual, non-Benford distributions. The Benford distribution is rejected for the first digits of the BoP statistics for Euro-adopting countries after these join the Euro zone. These findings confirm the viability of the intuition developed by the simple model in Section 3. However, we do not find general results for countries that were de facto more open to capital flows. This may be due to the fact that we are unable to capture the extent of their vulnerability very finely; but for some subgroups of countries (for example those with fixed exchange rate regimes and relatively high de facto openness) we still get very strong rejections of the null hypothesis. The interesting finding on the fixed exchange rate regime countries may show that the public is more readily misinformed by governments when there is a larger scope for misinformation - for example when the market prices aggregating private information are missing. We find also evidence that countries with weak institutions provide data that are non-Benford distributed; moreover, it seems that they are not driving our main results in Sections 6.1-6.4. 


\section{Conclusions}

In this paper we took a glimpse at the dark side of the moon of government statistics. We conclude that country-quarter pairs that correspond to economic situations in which countries would have higher incentives to misinform investors lead indeed to different distributions of the corresponding first digits of the balance of payments data than Benford's distribution, while for other country-quarter pairs the data conforms to this distribution. This may of course be because there is a different underlying process that rules the generation of balance of payments distributions in country-quarter pairs for vulnerable countries, which we think is unlikely. It may be, however, simply that such countries will at times falsify their balance of payments data in these quarters. This partial evidence on the strategic character of misreporting points out that models in which governments emit public signals (for example, the discussion on central bank transparency) should seriously consider the possibility that this signal may be at times intentionally misleading. On the policy side, this paper calls for the need to establish independent statistical agencies akin to that of independent central bankers. Some countries that failed in having such an independent agency, like Argentina or Greece, were caught red handed in altering economic data that they disseminated to the public. The possible welfare implications of misinformation should also be investigated; the actions of Argentina or Greece show that economic gains to governments, even if short-run, may exist and be substantial. In further work we would like to extend our analysis to fiscal data from many data sets.

\section{References}

Angeletos, George-Marios and Alessandro Pavan, "Efficient Use of Information and Social Value of Information," Econometrica, 2007, 75 (4), 1103-1142.

_ , Christian Hellwig, and Alessandro Pavan, "Signaling in a Global Game: Coordination and Policy Traps," Journal of Political Economy, 2006, 114 (3), 452-484.

Aumann, Robert J., Michael B. Marschler, and Richard E. Stearns, Repeated Games With Incomplete Information, MIT Press, 1995.

Benabou, Roland and Guy Laroque, "Using privileged information to manipulate markets: insiders, gurus and credibility," The Quarterly Journal of Economics, 1992, 107 (3), 921-958.

Benford, Frank, "The law of anomalous numbers," Proceedings of the American Philosophy Society, 1938, $78,551-572$.

Crawford, Vincent and Joel Sobel, "Strategic Information Transmission," Econometrica, 1982, 50, 14311451.

Curcuru, Stephanie E., Charles P. Thomas, and Francis E. Warnock, "Current Account Sustainability and Relative Reliability," Technical Report, in: Jeffrey Frankel and Christopher Pissarides (eds.), NBER International Seminar on Macroeconomics 2008, April 2009. 
Diaconis, Persi, “The distribution of leading digits and uniform distribution mod 1," The Annals of Probability, 1977, 5, 359-364.

Gelos, R. Gaston and Shang-Jin Wei, "Transparency and international investor behavior," Journal of Finance, 2005, 60 (6), 2987-3020.

Gonzalez-Garcia, Jesus and Gonzalo Pastor, “Benford's Law and Macroeconomic Data Quality,” Working Paper WP/09/10, International Monetary Fund 2009.

Heinemann, Frank and Gerhard Illing, "Speculative attacks: unique equilibrium and transparency," Journal of International Economics, 2002, 58, 429-450.

Hill, Theodore P., "Base-invariance implies Benford's law," Proceedings of the American Mathematical Society, 1995, 123, 887-895.

_ , "A statistical derivation of the significant-digit law," Statistical Science, 1995, 10, 354-363.

Holewijn, Piet J., "On the uniform distribution of sequences of random variables," Zeitschrift für Wahrscheinlichkeitstheorie und Verwandte Gebiete, 1969, 14, 89-92.

IMF, "Annual report on exchange arrangements and exchange restrictions," Technical Report 1989-2008.

_ , "Balance of Payments Manual,” Technical Report 1993.

_ , "Release of PricewaterhouseCoopers Report on the National Bank of Ukraine," News Brief 00/26 2000.

Janvresse, Elise and Thierry de la Rue, "From uniform distributions to Benford's law," Journal of Applied Probability, 2004, 41, 1203-1210.

Kaufmann, Daniel, Aart Kraay, and Massimo Mastruzzi, "Govenance Matters VIII: Aggregate and Individual Governance Indicators, 1996-2008,” Research Working Paper 4978, World Bank Policy 2009.

Kaynar, Bahar, Arno Berger, Theodore P. Hill, and Ad Ridder, "Finite-State Markov Chains obey Benford's Law,” Working Paper TI 2010-030/4, Tinbergen Institute 2010.

Krugman, Paul, "A model of balance of payments crises," Journal of Money, Credit and Banking, 1979, 11, 311-325.

Kydland, Finn E. and Edward C. Prescott, "Rules rather than discretion: The inconsistency of optimal plans," Journal of Political Economy, 1977, 85, 473-491.

Lane, Phillip and Gian-Maria Milesi-Ferretti, "The External Wealth of Nations Mark II: Revised and Extended Estimates of Foreign Assets and Liabilities, 1970-2004," Journal of International Economics, 2007, $73,223-250$.

Ley, Eduardo, “On the Peculiar Distributrion of the U.S. Stock Indices’ Digits," The American Statistician, 1996, 50, 311-313. 
Morris, Stephen and H.S. Shin, "The Social Value of Public Information,” American Economic Review, 2002, 92, 1521-1534.

Newcomb, Simon, "Note on the frequency of use of the different digits in natural numbers," American Journal of Mathematics, 1881, 4, 39-40.

Nigrini, Mark, “Taxpayer compliance application of Benford's law," Journal of the American Taxation Association, 1996, 18, 72-92.

_ , "I've got your number: How a mathematical phenomenon can help CPAs uncover fraud and other irregularities," Journal of Accountancy, May 1999.

Nye, John and Charles Moul, "The political economy of numbers: on the application of Benford's law to international macroeconomic statistics," The B.E. Journal of Macroeconomics, 2007, 7 (1), article 17.

Obstfeld, Maurice, "Rational and self-fulfilling balance of payments crises.," American Economic Review, 1986, 76, 72-81.

_ , "Models of Currency Crises with Self-Fulfilling Features.," European Economic Review, 1996, 40, 10371047.

Pinkham, Roger S., "On the distribution of first significant digits," The Annals of Mathematical Statistics, 1961, 32, 1223-1230.

Reinhart, Carmen and Kenneth Rogoff, This Time is Different, Princeton University Press, 2009.

Rivoirard, Vincent and Gilles Stoltz, Statistique en action, Vuibert, 2009.

Sandleris, Guido, "Sovereign defaults: Information, investment and credit," Journal of International Economics, 2008, 76, 267-275.

Sbracia, Massimo and Andrea Zaghini, "Expectations and information in second-generation currency crises models," Economic Modelling, 2001, 18, 203-222.

Schürger, Klaus, "Extensions of Black-Scholes processes and Benford's law," Stochastic Processes and their Applications, 2008, 118, 1219-1243.

Standard and Poor's, “Sovereign Credit Ratings: a Primer,” Technical Report 2008.

Stein, Jeremy C., "Cheap Talk and the Fed: A Theory of Imprecise Policy Announcements," American Economic Review, 1989, 79 (1), 32-42.

Tarashev, Nikola A., "Speculative Attacks and the Information Role of the Interest Rate," Journal of the European Economic Association, 2007, 5, 1-36.

van der Vaart, Aad, Asymptotic Statistics, Cambridge University Press, 1998.

Varian, Hal R., "Benford's Law,” The American Statistician, 1972, 26, 65-66.

Young, Alwyn, “The African Growth Miracle,” mimeo, London School of Economics 2009. 


\section{Newspaper articles}

Financial Times (1999). Andrew Jack.“Loans may hang on report disclosure.” 2 July 1999.

Financial Times (2001, via FT.Com). R. Bream, J. Blitz and P. Norman."Doubts over way Italy qualified for euro." 4 November 2001.

Financial Times (2003). Alan Ruskin.“A truer measure of China’s trade surplus.” 30 October 2003.

Financial Times (2006). Christopher Condon. "The Speech that sparked rioting.” 20 September 2006.

Financial Times (2007). Stefan Wagstyl. “Lies haunt a reformer’s grip on power.” 25 October 2007.

Financial Times (2008). J. Webber. “As prices surge, Argentines cry foul over the official figures.” 9 June 2008.

Financial Times (2009). Bernie McSherry. “Investors have to be sure that statistics do not lie.” 16 July 2009.

Financial Times (2010). T. Barber and K. Hope. "Brussels attacks Greece over false data.” 13 January 2010.

International Monetary Fund Press Release No. 03/98 (2003). "IMF Executive Board Reviews Noncomplying Disbursement to Chad." 26 June 2003.

International Monetary Fund Public Information Notice No. 05/121 (2005). "IMF Executive Board Concludes 2005 Article IV Consultation with The Gambia.” 8 September 2005.

International Monetary Fund Press Release No. 06/94 (2006). "IMF Executive Board Approves USD 46.3 Million PRGF Arrangement for Sierra Leone” 10 May 2006.

Reuters News (2005). “Egypt 2004/5 C/A surplus slips due to import surge.” 13 October 2005.

The Wall Street Journal (2004). J. W. Miller. "Obscure EU statistics bureau may be getting a higher profile." 10 November 2004.

The Wall Street Journal (2008, online version). M. O'Grady. "Argentina Impoverishes Itself Again; What happens when government meddles with private wealth.” 3 November 2008. 


\section{A Incentives to misreport economic data: Proofs of the stated results}

We provide in this section the proofs of the results stated in Section 3 in the main text.

Existence and properties of the equilibrium with lying. Investors wish to maximize their ex-ante return from investment, hence they will allocate capital $\bar{K}$ so that the marginal return from investment in all countries is equalized, no matter whether they declare a Good or Bad state of the world. This leads, on the one hand (when $C_{i} \equiv C$ for all countries $i$ ), to the stated fact that the capital $K_{i}\left(s_{i}, \tilde{\rho}\right)$ invested in country $i$ does not depend on $i$ but solely on $s_{i}$ and $\tilde{\rho}$; on the other hand, that returns are equal for countries declaring Good or Bad states as in (3). As $\tilde{\rho} \geqslant \hat{\rho}$, investment return in countries that declare a Good state of the world is lower than if all these countries would in fact be enjoying a Good state. The equality (3) needs to be a feature of any efficient allocation, as then investors are indifferent between investing marginally more in one group of countries than in the other. Note also that (3) indicates that the ratio $\Xi$ of $K(g, \tilde{\rho})$ to $K(b, \tilde{\rho})$ equals, at equilibrium,

$$
\begin{array}{r}
\Xi(\tilde{\rho}, \varphi)=\frac{K(b, \tilde{\rho})}{K(g, \tilde{\rho})}=\left(\frac{\hat{\rho} A(g)+\varphi(1-\hat{\rho}) A(b)}{\tilde{\rho} A(b)}\right)^{1 /(\alpha-1)}=\left(1-\frac{\hat{\rho}(A(g)-A(b))}{\hat{\rho} A(g)+\varphi(1-\hat{\rho}) A(b)}\right)^{1 /(1-\alpha)} \\
\text { with } \Xi(\tilde{\rho}, \varphi)<1 .
\end{array}
$$

Given this and the global mobile capital supply $\bar{K}$, we can find the capital values $K(b, \tilde{\rho})$ and $K(b, \tilde{\rho})$, the stock of capital in countries declaring a Bad and Good states respectively. Indeed, the market clearing condition in the capital market is

$$
\int_{[0,1]}(K(b, \tilde{\rho})-C) \mathbb{I}_{\left\{d_{i}=b\right\}} \mathrm{d} i+\int_{[0,1]}(K(g, \tilde{\rho})-C) \mathbb{I}_{\left\{d_{i}=g\right\}} \mathrm{d} i=(1-\tilde{\rho}) K(b, \tilde{\rho})+\tilde{\rho} K(g, \hat{\rho})-C=\bar{K}
$$

After some algebra and by substituting the equality $\tilde{\rho}=\hat{\rho}+\varphi(1-\hat{\rho})$, one can show that

$$
K(g, \hat{\rho})=\frac{\bar{K}+C}{\hat{\rho}+(1-\hat{\rho})(\varphi+\Xi-\varphi \Xi)} .
$$

We now show that $\varphi+\Xi-\varphi \Xi$ increases as $\varphi$ increases, which entails that $K(g, \hat{\rho})$ then decreases; the derivative of the considered expression with respect to $\varphi$ indeed equals $(1-\Xi)+(1-\varphi) \partial \Xi / \partial \varphi$, where both summands are positive as $\Xi<1$ and as $\Xi(\tilde{\rho}, \varphi)$ is increasing in $\varphi$ as can be seen from its closed-form expression. The interpretation is that as the fraction $\varphi$ of liars among countries with $s_{i}=b$ increases, the capital invested in countries declaring a high state of the world declines; this is because the return from investment in these countries falls.

Countries. At equilibrium, in the interior when $\varphi \in(0,1)$, countries are indifferent between lying and reporting truthfully the state of the world when their state is $s_{i}=b$. When making their decision, 
they do not know $\hat{\rho}$, hence their strategies can depend only on the information they have, i.e., on their own state of the world and the knowledge of the distribution of $\rho$. The first step is to analyze the payoffs of countries given in (2). Since we showed above that the functions $K_{i}$ giving the invested capitals did not depend on $i$, the same is true for the return functions $R_{i}$, which we will simply denote by $R$ in the sequel (the same holds for the expected returns $E R$ ). A country that has a Good state of the world $s_{m}=g$ will not declare a Bad state of the world; it would not only receive less capital from investors (as these make decisions based on declarations only and $K(g, \tilde{\rho})<K(b, \tilde{\rho})$ ), but also obtain a punishment. In other words, $R(b, g, \tilde{\rho})-R(g, g, \tilde{\rho})<0$. To analyze the incentive of countries with a Bad state of the world, we introduce the gap $\Delta(\tilde{\rho})=R(b, g, \tilde{\rho})-R(b, b, \tilde{\rho})$. Because of the antagonist effects of the punishment and the increase of invested capital when lying, this gap can in principle be positive, negative, or null.

Lemma 1. The gap in payoffs $\Delta(\tilde{\rho})$ is strictly decreasing in $\varphi$, i.e., decreases as the proportion $\varphi$ of liars is higher.

Proof. By definition of $\Xi$ and by (2), one gets that

$$
\Delta(\tilde{\rho})=A(b)\left((1-\alpha)(K(g, \tilde{\rho}))^{\alpha}\left(1-\Xi^{\alpha}\right)+\alpha C(K(g, \tilde{\rho}))^{\alpha-1}\left(1-\Xi^{\alpha-1}\right)\right) ;
$$

but since $\Xi$ increases as $\varphi$ increases (as can be seen from its closed-form expression) and since the above expression of $\Delta$ shows that it is a decreasing function of $\Xi$, the conclusion follows.

The higher the $\varphi$, the more liars given any $\hat{\rho}$, and the lower the capital that is invested in countries declaring $g$. Hence, there is a lower incentive to lie when the country in fact has a true state $b$. The following result is immediate by taking expectations (with respect to $\tilde{\rho}$ ) in the lemma.

Proposition 6. The expected gap $E R(b, g)-E R(b, b)$ is strictly decreasing in $\varphi$.

In particular, the mapping $\varphi \mapsto E R(b, g)-E R(b, b)$ is one-to-one. If there exists a $\varphi \in(0,1)$ such that $E R(b, g)-E R(b, b)=\Phi$ then this $\varphi$ is unique and the investors can deduce it from the value of $\Phi$; in particular, they are then able to precisely deduce the value of $\hat{\rho}$ given the fraction of countries $\tilde{\rho}$ declaring a Good state of the world: since the strategies of countries do not depend on $\hat{\rho}$, unknown to the countries when making the declarations, and since $\tilde{\rho}=\hat{\rho}+\varphi(1-\hat{\rho})$, the investors can learn the true $\hat{\rho}=(\tilde{\rho}-\varphi) /(1-\varphi)$. If $\varphi \in(0,1)$ then countries with $s_{m}=b$ are indifferent between declaring truthfully and misreporting. If $\varphi=1$ then all countries with $s_{m}=b$ find it more profitable to lie than to declare truthfully despite the expected punishment $\Phi$. If $\varphi=0$ then all countries declare truthfully; this is the case whenever $\Phi$ is large enough (larger than the expected gap in payoffs). All in all, we showed that countries have the correct incentives given $\Phi$ and the parameters of the environment (the stock of mobile capital, productivity differences between the Good and the Bad state, etc.).

Proof of Proposition 1. When $\varphi \in(0,1)$ at equilibrium, one has $E R(b, g)-E R(b, b)=\Phi$; but Proposition 6 precisely stated that $\varphi \mapsto E R(b, g)-E R(b, b)$ is decreasing, so that the inverse mapping $\Phi \mapsto \varphi$ of what happens at equilibrium is decreasing as well. 
Comparative statics (proofs of Propositions 2-5). The simplest proof of the one of Proposition 5, since this result does not rely on a partitioning of the countries into two groups.

Proof of Proposition 5. Countries make the choice whether to report $g$ or $b$ based on the properties of the distribution of $\rho$, as at the moment of making the decision they do not know the actual fraction $\hat{\rho}$ of countries enjoying a Good state. Hence, at a given equilibrium (i.e., for a given value of $\varphi$ ), a constant fraction $\varphi$ of those that obtained a $b$ state lies and the fraction of liars in the entire population is $\varphi(1-\hat{\rho})$, which is decreasing in $\hat{\rho}$ as stated.

The framework for all other propositions to be proved is to split countries into two groups, with respective proportions $\xi$ and $1-\xi$, for which it is easy to see - following the same lines as abovethat at equilibrium they share the same characteristics; this defines a common invested capital function $\underline{K}$ or $\bar{K}$ in each group, a common expected return function $\underline{E R}$ or $\overline{E R}$, a common cheating rate $\underline{\varphi}$ or $\bar{\varphi}$, and so forth. The observed proportion of countries declaring a Good state is now

$$
\tilde{\rho}=\hat{\rho}+(1-\hat{\rho})(\xi \underline{\varphi}+(1-\xi) \bar{\varphi})
$$

the respective proportions of countries not truthfully declaring their states in each group are given by

$$
\frac{(1-\hat{\rho}) \underline{\varphi}}{\hat{\rho}+(1-\hat{\rho}) \underline{\varphi}} \quad \text { and } \quad \frac{(1-\hat{\rho}) \bar{\varphi}}{\hat{\rho}+(1-\hat{\rho}) \bar{\varphi}}
$$

As above, at equilibrium, investors can determine $\underline{\varphi}$ and $\bar{\varphi}$ and infer $\hat{\rho}$ from $\tilde{\rho}$.

Proof of Proposition 2. We are interested in the behavior of the countries when there is lying in equilibrium, i.e., when $\underline{\varphi}, \bar{\varphi} \in(0,1)$ at equilibrium; in this case, as follows from an immediate adaptation of the arguments above in each of the groups, $\underline{E R}(b, g)-\underline{E R}(b, b)=\underline{\Phi}$ and $\overline{E R}(b, g)-\overline{E R}(b, b)=\bar{\Phi}$, where each gap is still a decreasing function of the respective proportions $\varphi$ and $\bar{\varphi}$ of liars (see Proposition 6). The conclusion follows.

The proofs of the last two propositions are relegated due to their length to Appendix C, Section C.1; we provide here only a sketch of how these are constructed.

Sketch of proof of Proposition 3. In any equilibrium, investors demand that investment brings ex-ante the same return at the margin in either group of countries, irrespective of the declared state of the world. We use this condition and find that the following must be true: $\bar{\varphi}<\varphi$ if and only if $\bar{K}(g, \tilde{\rho})>$ $\underline{K}(g, \tilde{\rho})$ for at least one ${ }^{47}$ realization $\hat{\rho}$. To show that indeed $\bar{K}(g, \tilde{\rho})>\underline{K}(g, \tilde{\rho})$ we use that for equilibria in the interior, $\underline{E R}(b, g)-\underline{E R}(b, b)=\Phi$ and $\overline{E R}(b, g)-\overline{E R}(b, b)=\Phi$ must hold, take a difference of the two conditions and substitute.

\footnotetext{
${ }^{47}$ It can be shown that it will then hold for all realizations of $\hat{\rho}$.
} 
Sketch of proof of Proposition 4. We use a similar line of reasoning as in the above proof. From the ex-ante return equalization on any group of countries, it can be shown that the stated $\bar{\varphi}<\varphi$ holds if and only if $\bar{K}(b, \tilde{\rho}) / \underline{K}(b, \tilde{\rho})<\bar{K}(g, \tilde{\rho}) / \underline{K}(g, \tilde{\rho})$ for at least one realization $\hat{\rho}$. We use the same property of the interior equilibrium $\underline{E R}(b, g)-\underline{E R}(b, b)=\Phi$ and $\overline{E R}(b, g)-\overline{E R}(b, b)=\Phi$ to show that the right hand side of the equivalency condition holds.

\section{B Tables and figures}

They are reported in the following pages (one table or set of figures per page). 

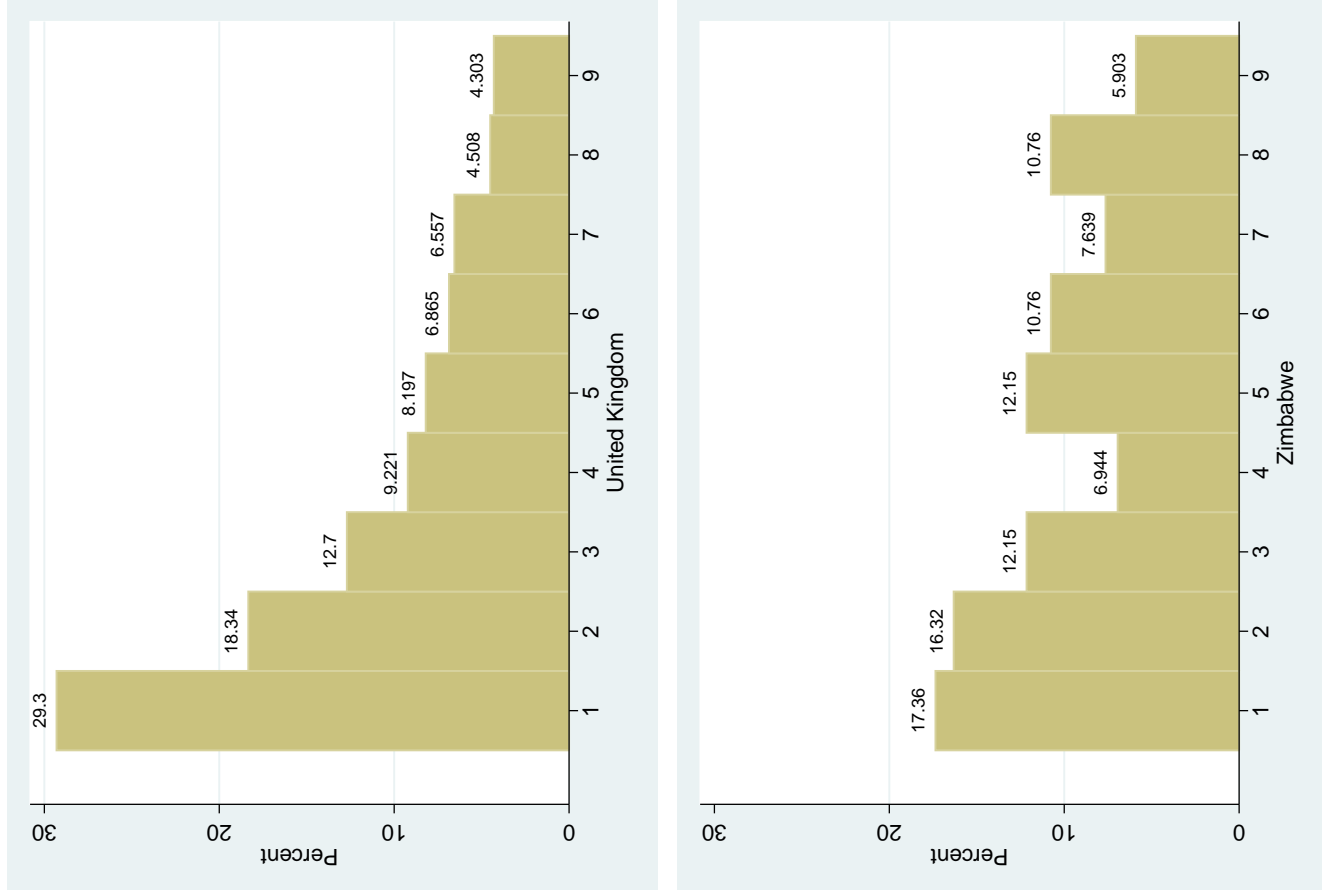

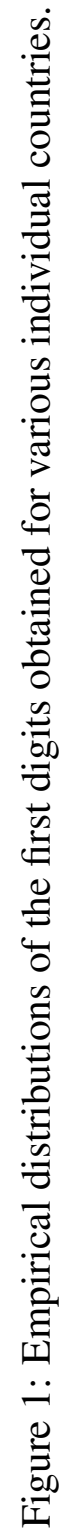
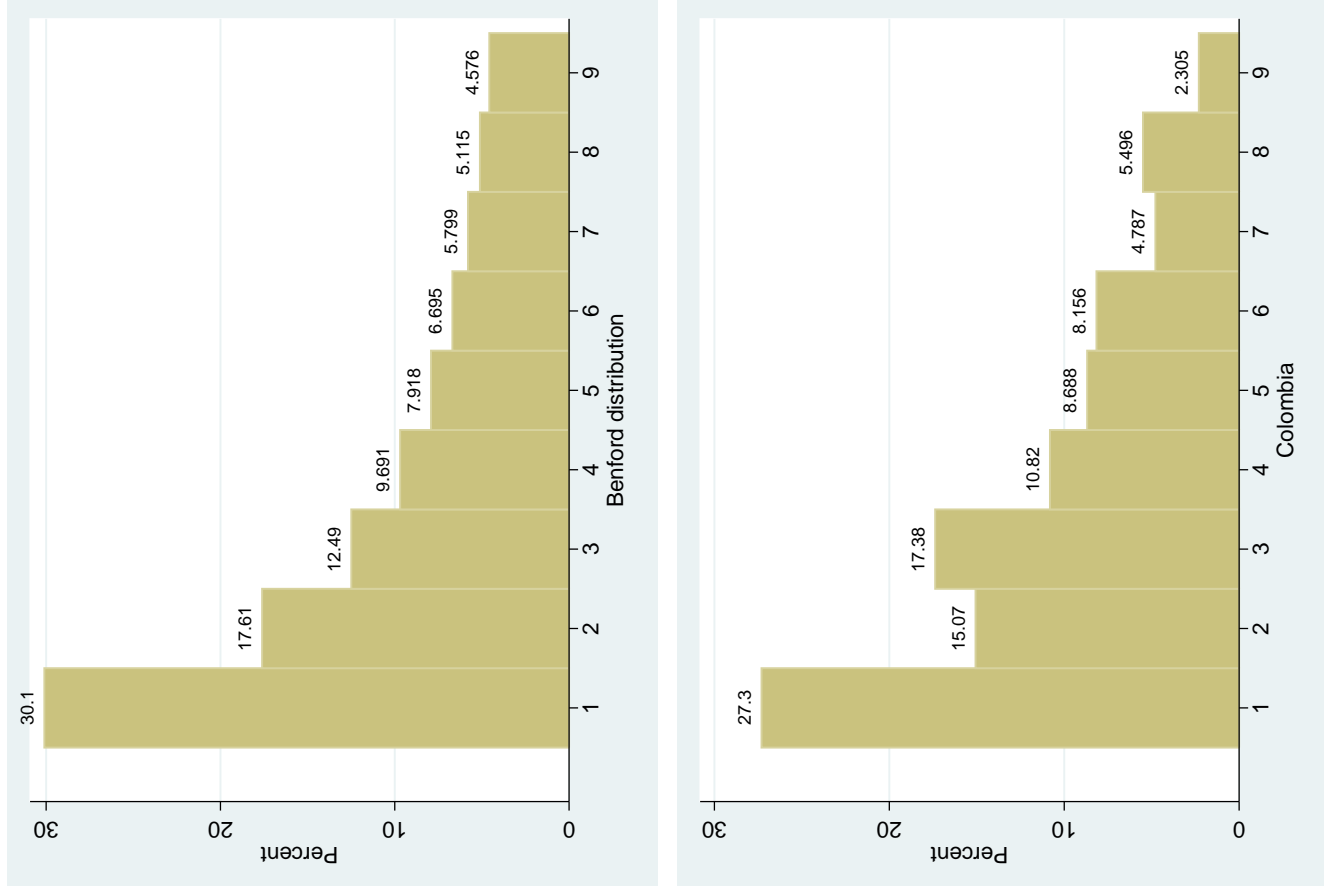


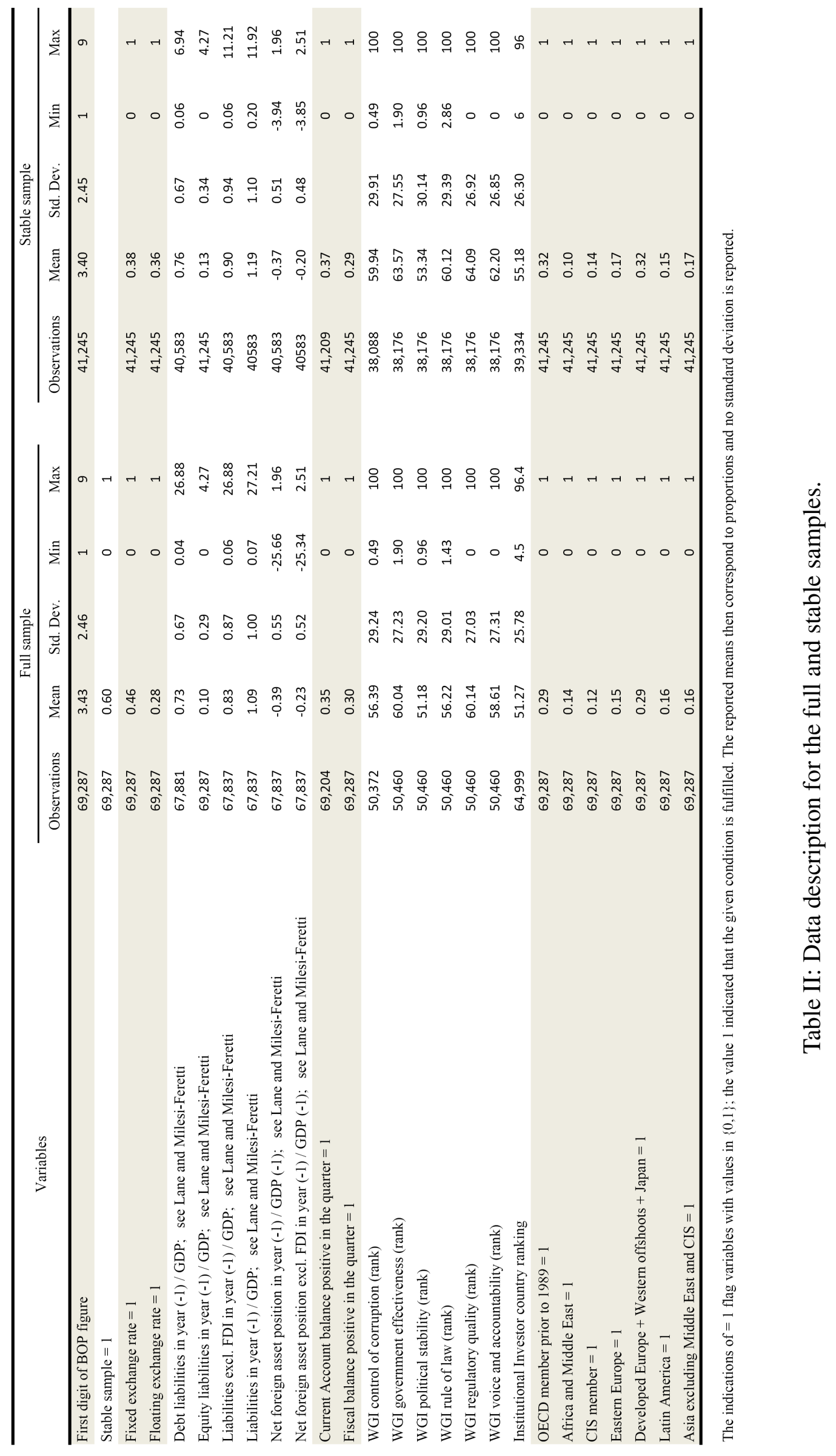




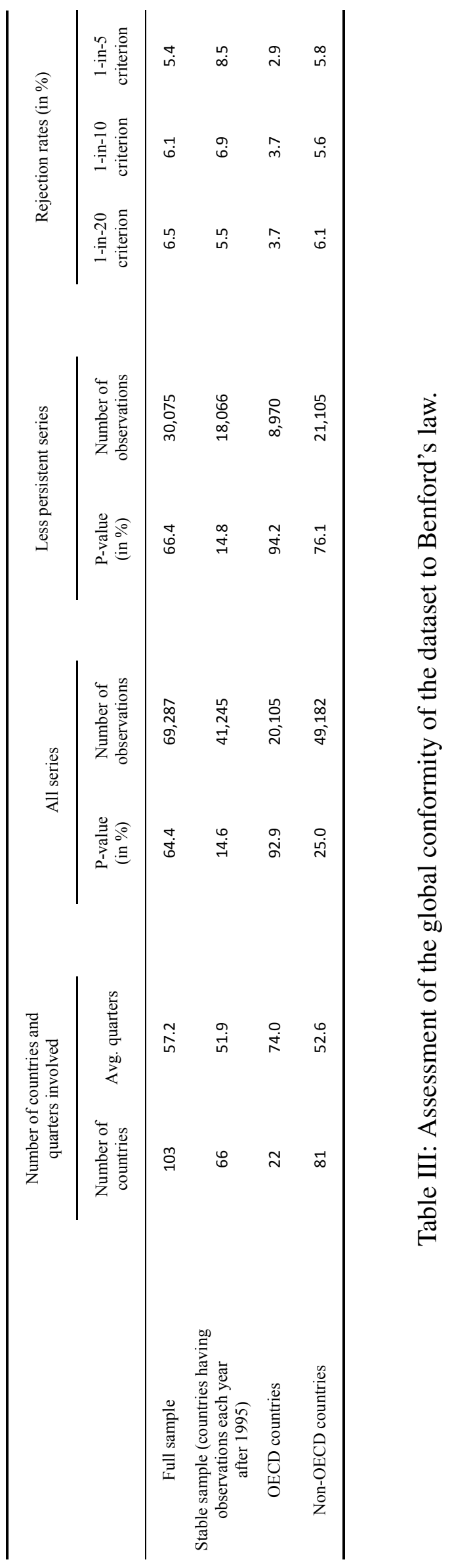



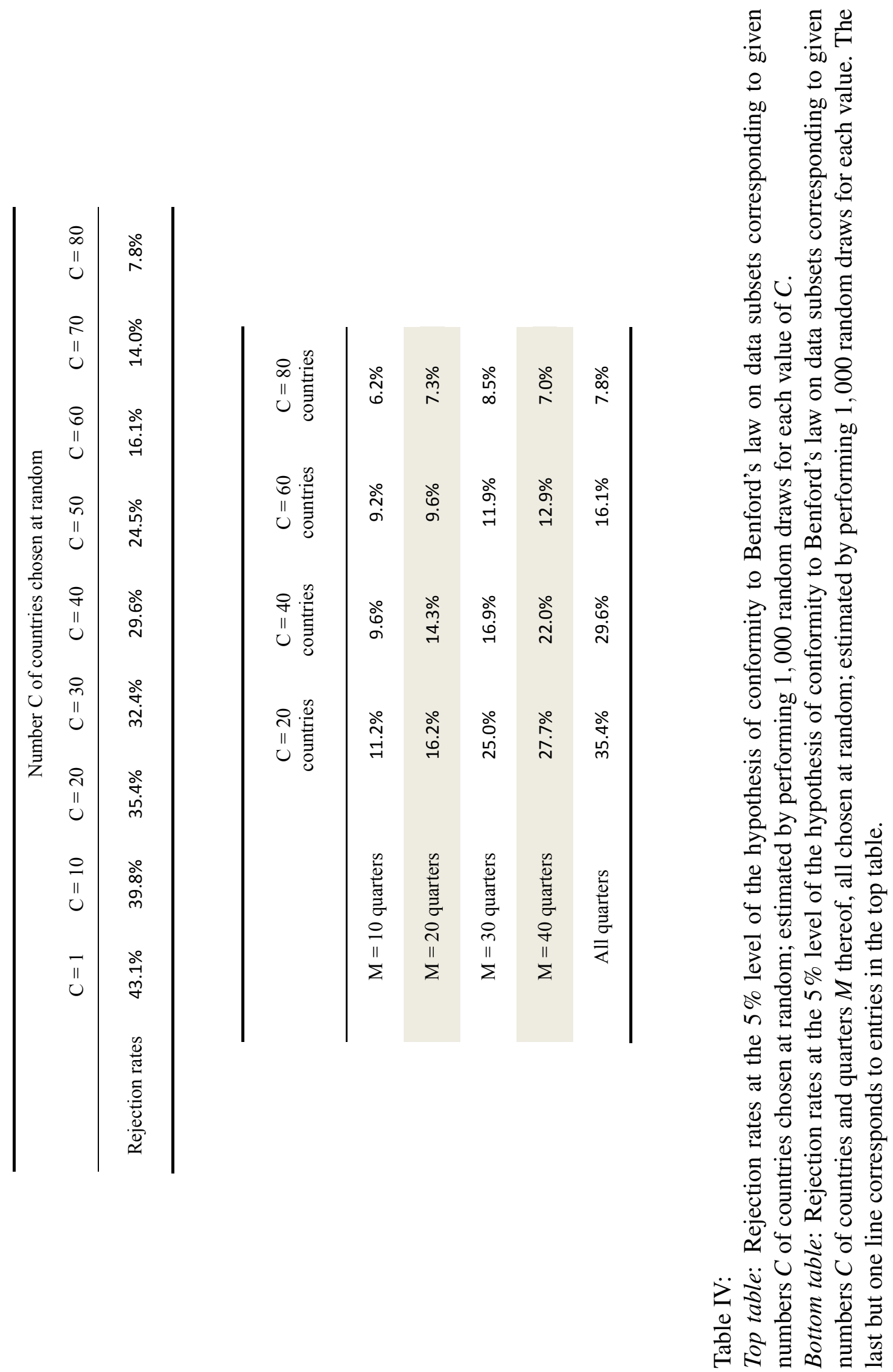


\begin{tabular}{ccccc}
\hline $\begin{array}{c}\text { Proportion } \\
f=10 \%\end{array}$ & \multicolumn{4}{c}{ Estimated quantiles (values given in \%) } \\
\hline $\mathrm{N}=1,200$ & $50 \%$ & $95 \%$ & $98 \%$ & $99 \%$ \\
$\mathrm{~N}=2,000$ & 7.0 & 6.3 & 7.2 & 8.0 \\
$\mathrm{~N}=5,000$ & 6.9 & 7.9 & 9.1 & 9.5 \\
$\mathrm{~N}=10,000$ & 6.9 & 7.8 & 9.0 & 9.8 \\
$\mathrm{~N}=30,000$ & 6.8 & 7.5 & 8.7 & 9.9 \\
$\mathrm{~N}=50,000$ & 6.8 & 7.7 & 9.7 & 9.5 \\
\hline & \multicolumn{5}{c}{ Conclusion: upper bounds } \\
\hline $\mathrm{N} \geq 2,000$ & 7.0 & 8.0 & 9.0 & 9.7 \\
\hline
\end{tabular}

Table V: Estimates of the quantiles of the distribution of the random variable $\bar{R}_{D, 1000}$ when $D$ is chosen at random according to Benford's law and $f$ equals $10 \%$; estimates were obtained by drawing $D$ at random 1,000 times and computing the associated values of $\bar{R}_{D, 1000}$. 


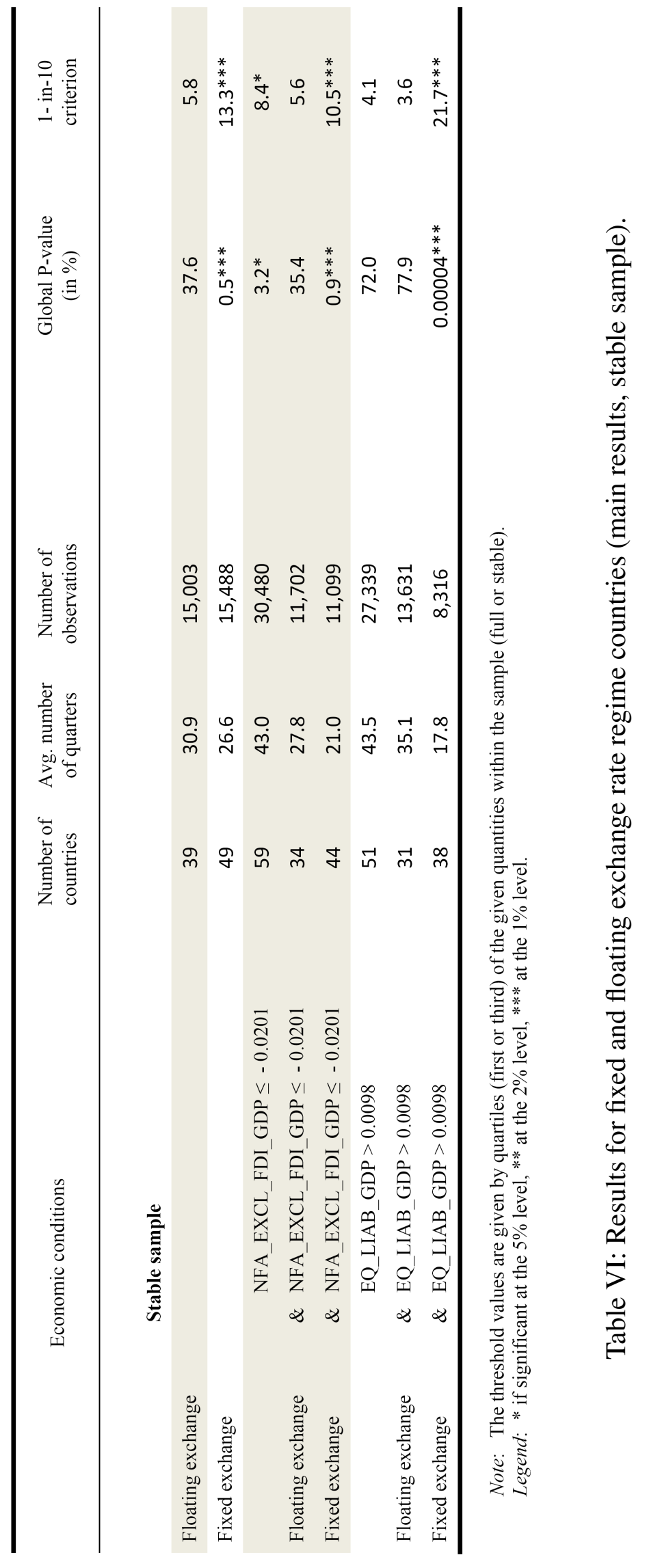




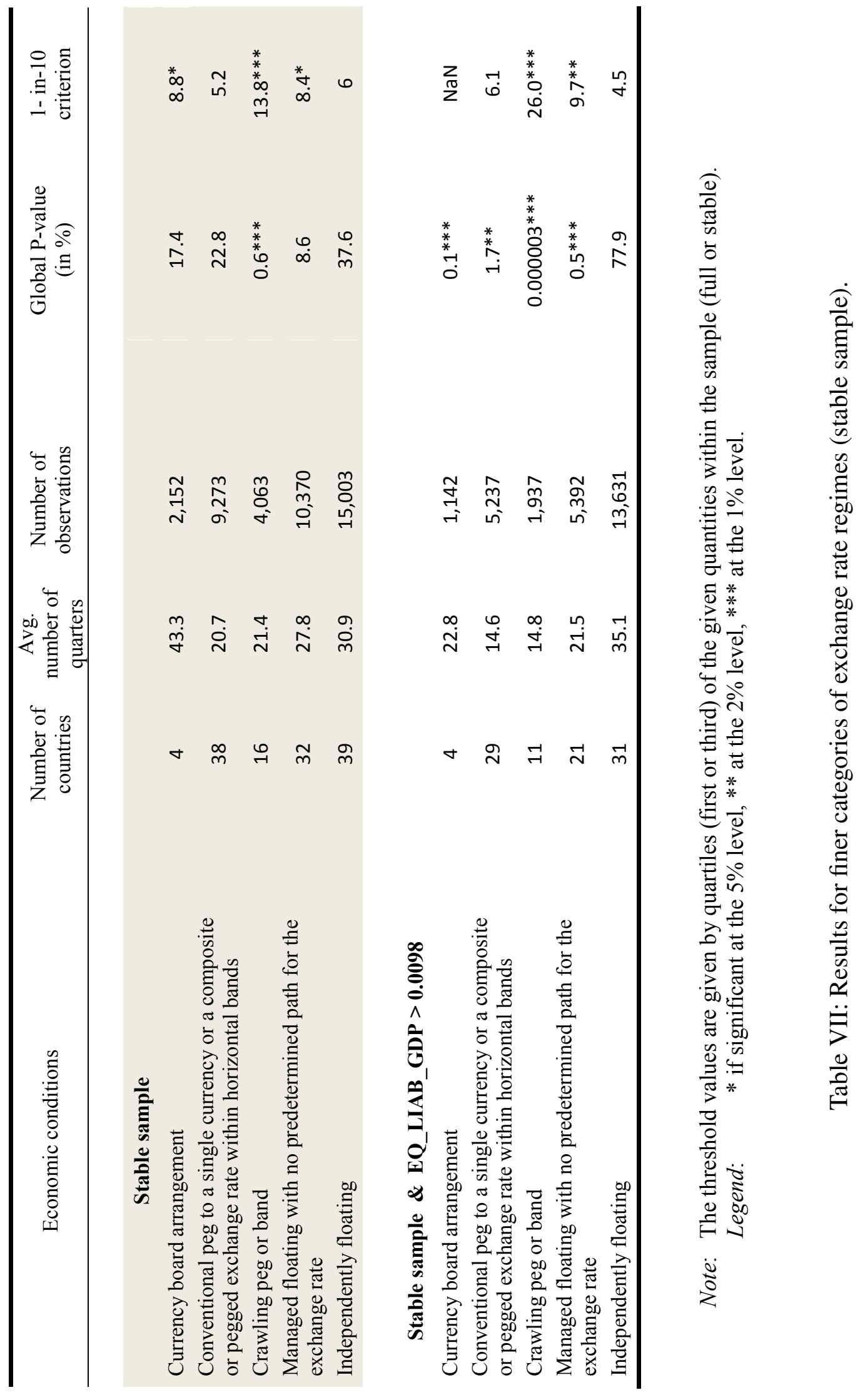




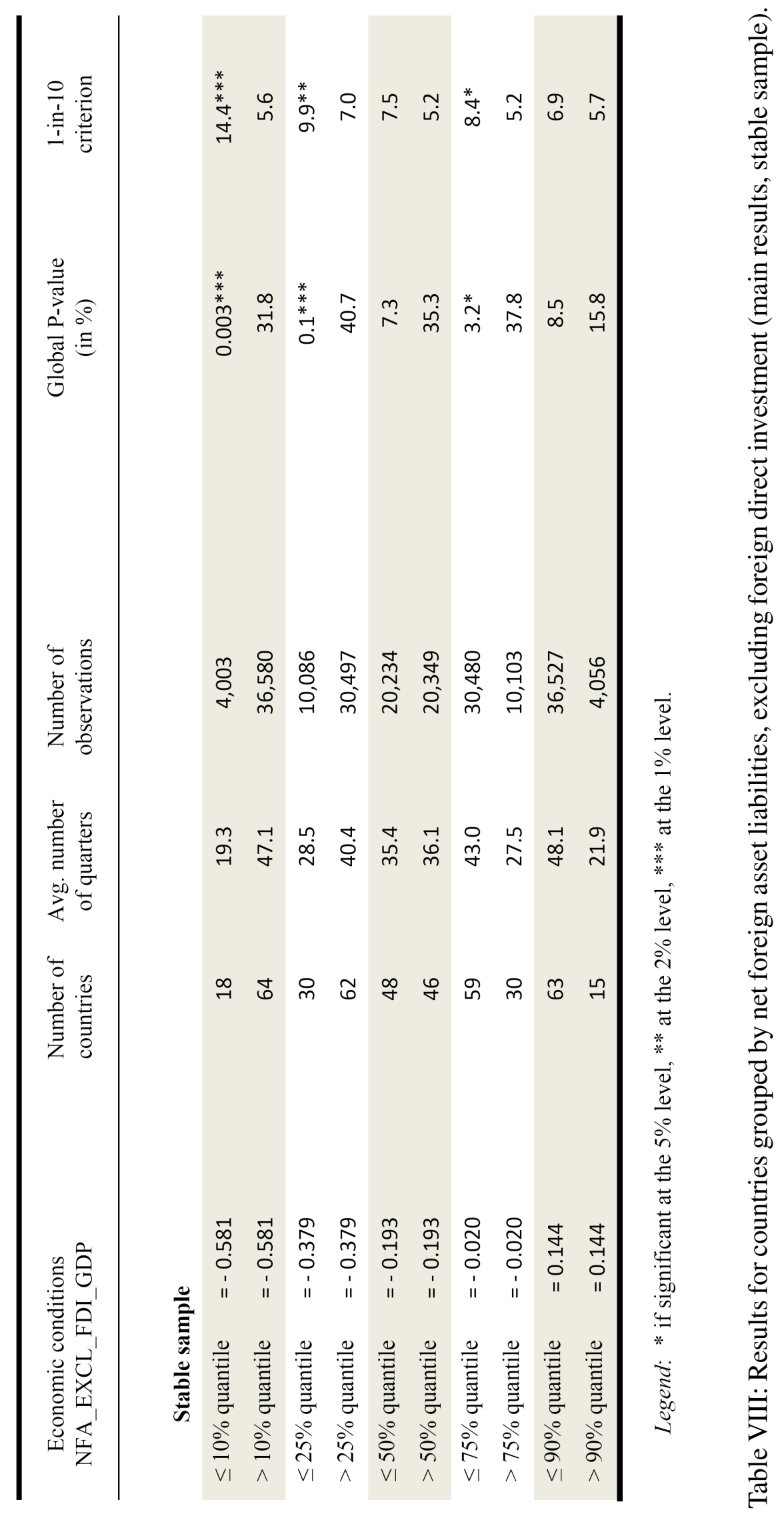




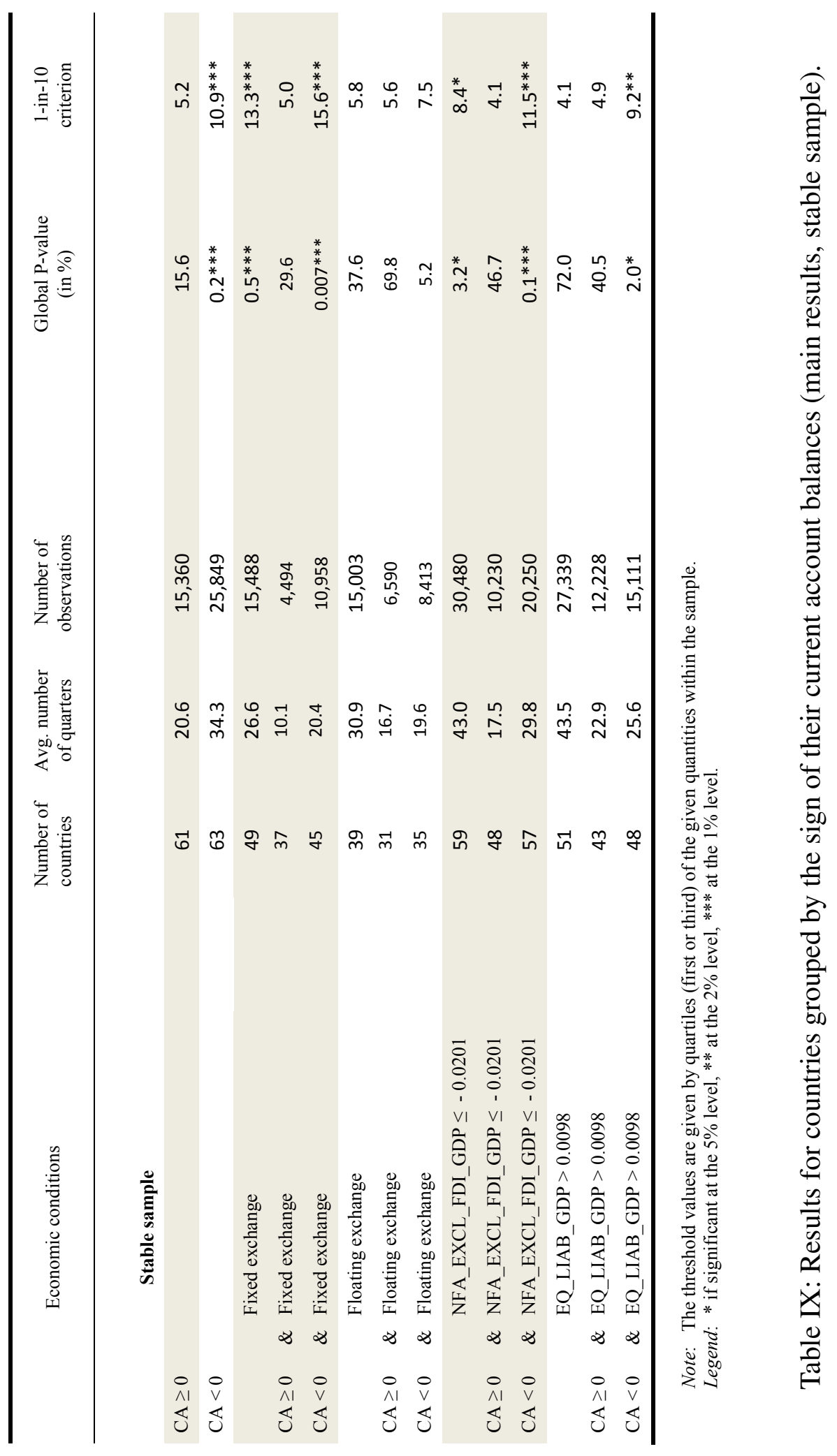




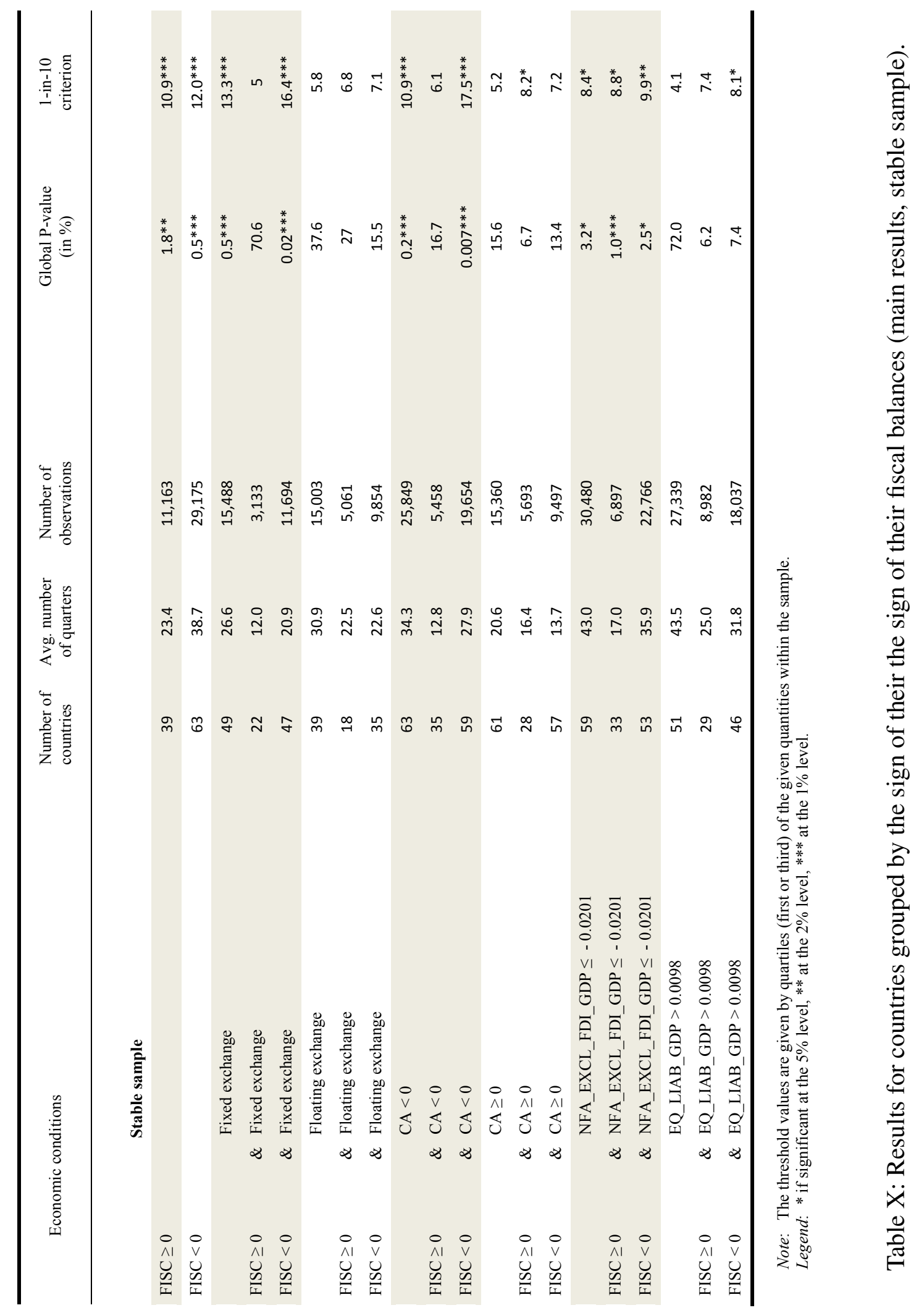




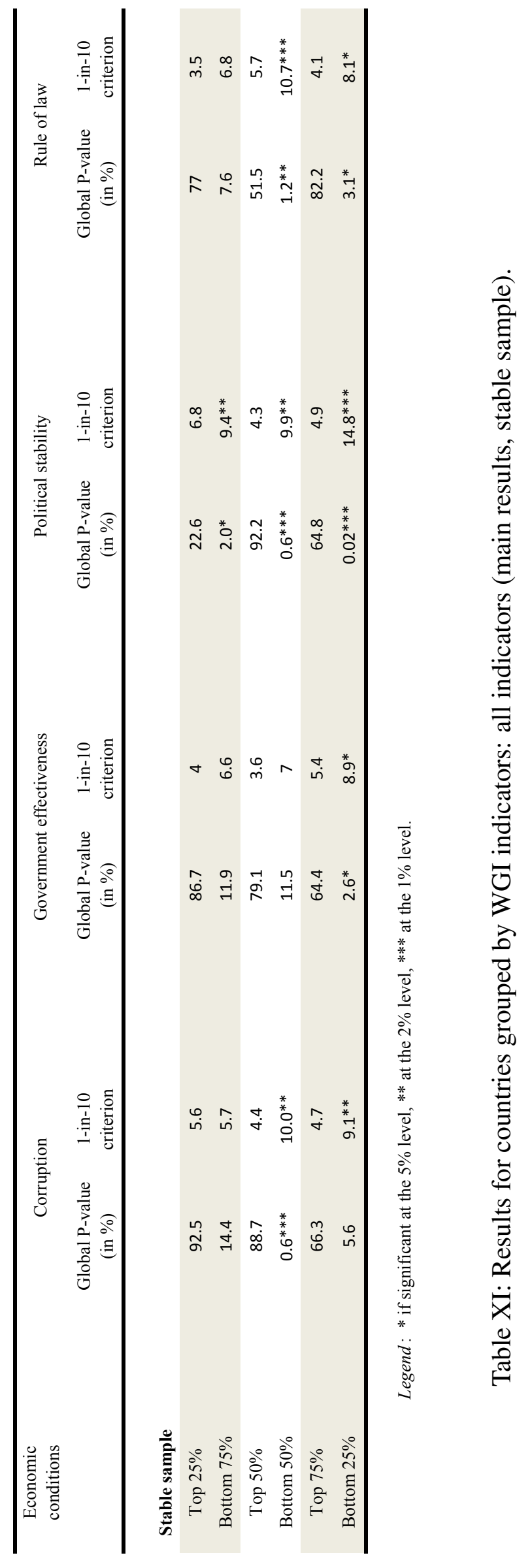




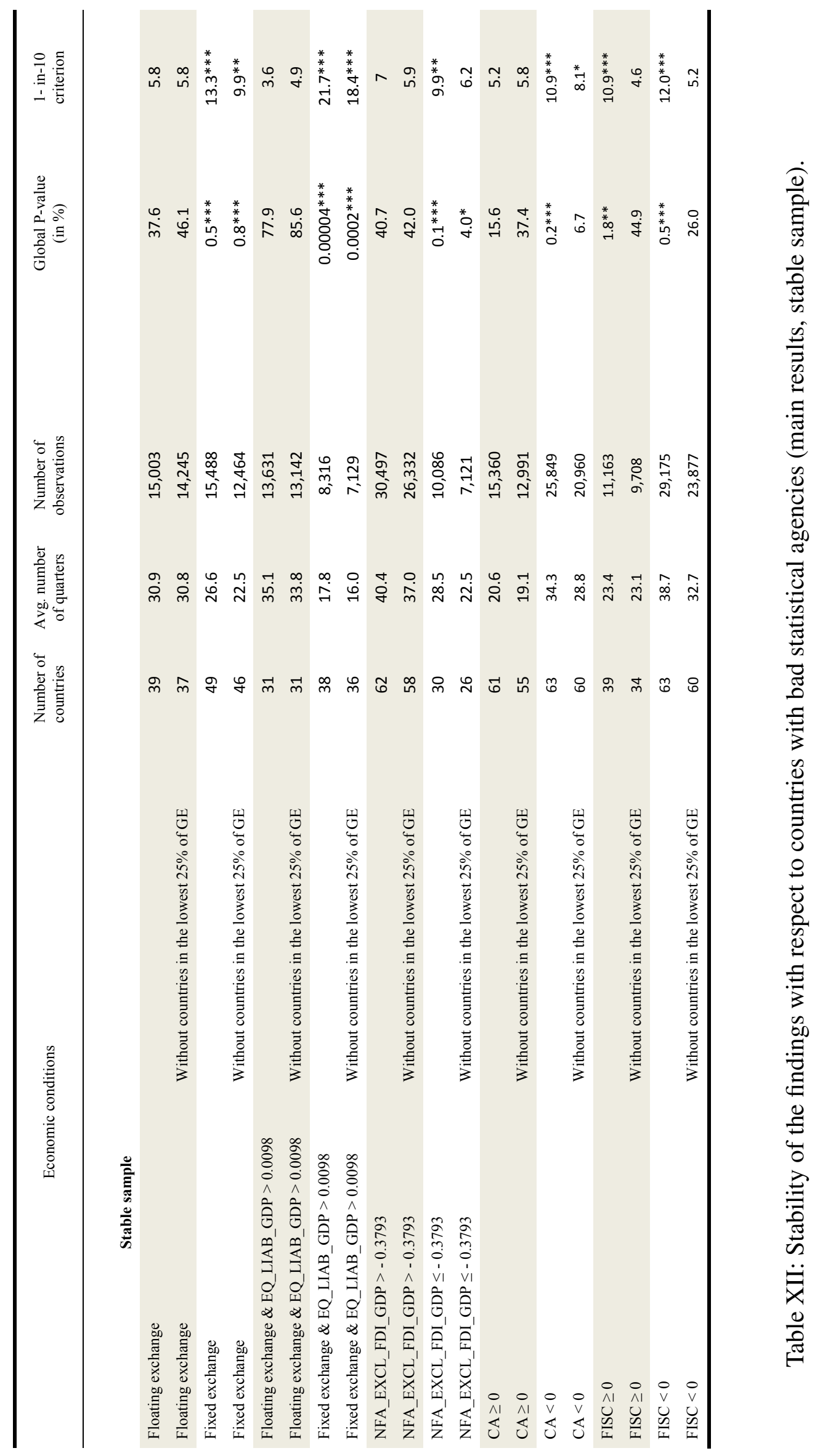





\section{Additional material - Working paper}

This is the companion working paper to the paper "Do countries falsify economic data strategically? Some evidence that they might." by Tomasz Michalski and Gilles Stoltz. It provides some technical details on the material alluded at in the main body of the text. It contains

- extended proofs (Section C.1);

- additional discussion of the Benford law and its application to macroeconomic sequences (Section C.2);

- list of countries included in the study (Section C.3);

- other results not discussed in the main text (Section C.4);

- more detailed tables of the main results for the stable sample; tables of results for the full sample and robustness checks (Section C.4.3).

\section{C.1 Extended proofs}

Proof of Proposition 3. In view of the respective proportions of liars stated above, the equality of returns to investors for countries declaring Good states in both groups, i.e., considering the left-hand side of (3), implies in particular that for all realizations $\hat{\rho}$,

$$
\begin{aligned}
& \alpha\left(\frac{\hat{\rho}}{\hat{\rho}+\bar{\varphi}(1-\hat{\rho})} A(g)+\frac{\bar{\varphi}(1-\hat{\rho})}{\hat{\rho}+\bar{\varphi}(1-\hat{\rho})} A(b)\right)(\bar{K}(g, \tilde{\rho}))^{\alpha-1} \\
& \quad=\alpha\left(\frac{\hat{\rho}}{\hat{\rho}+\underline{\varphi}(1-\hat{\rho})} A(g)+\frac{\underline{\varphi}(1-\hat{\rho})}{\hat{\rho}+\underline{\varphi}(1-\hat{\rho})} A(b)\right)(\underline{K}(g, \tilde{\rho}))^{\alpha-1} .
\end{aligned}
$$

Since

$$
\varphi \longmapsto \frac{\hat{\rho}}{\hat{\rho}+\varphi(1-\hat{\rho})} A(g)+\frac{\varphi(1-\hat{\rho})}{\hat{\rho}+\varphi(1-\hat{\rho})} A(b)=\left(1+\frac{\hat{\rho}(A(g)-A(b))}{(\hat{\rho}+\varphi(1-\hat{\rho})) A(b)}\right) A(b)
$$

is a decreasing function of $\varphi$ (no matter the value of $\hat{\rho}$ ) and since $\alpha-1<0$, the above stated equality entails that $\bar{\varphi}<\underline{\varphi}$ if and only if $\bar{K}(g, \tilde{\rho})>\underline{K}(g, \tilde{\rho})$ for at least one ${ }^{48}$ realization $\hat{\rho}$. We will prove that the latter condition is indeed met by showing that for some increasing function $\psi$,

$$
\mathbb{E}[\psi(\bar{K}(g, \tilde{\rho}))-\psi(\underline{K}(g, \tilde{\rho}))]>0
$$

\footnotetext{
${ }^{48}$ It can be shown that it will then hold for all realizations of $\hat{\rho}$.
} 
where expectations are taken with respect to $\hat{\rho}$ (which is distributed according to $\rho$ ). To do so, we study the incentives of countries. At equilibrium, we have

$$
\left\{\begin{array}{l}
\mathbb{E}[\underline{\Delta}(\tilde{\rho})]=\underline{E R}(b, g)-\underline{E R}(b, b)=\Phi, \\
\mathbb{E}[\bar{\Delta}(\tilde{\rho})]=\overline{E R}(b, g)-\overline{E R}(b, b)=\Phi ;
\end{array}\right.
$$

we now study the difference

$$
\mathbb{E}[\bar{\Delta}(\tilde{\rho})]-\mathbb{E}[\underline{\Delta}(\tilde{\rho})]=\overline{E R}(b, g)-\overline{E R}(b, b)-\underline{E R}(b, g)+\underline{E R}(b, b)=0,
$$

which can be rewritten as

$$
\begin{aligned}
& \mathbb{E}\left[A(b)\left((1-\alpha)\left((\bar{K}(g, \tilde{\rho}))^{\alpha}-(\bar{K}(b, \tilde{\rho}))^{\alpha}\right)+\alpha \bar{C}\left((\bar{K}(g, \tilde{\rho}))^{\alpha-1}-(\bar{K}(b, \tilde{\rho}))^{\alpha-1}\right)\right)\right. \\
& \left.\quad-A(b)\left((1-\alpha)\left((\underline{K}(g, \tilde{\rho}))^{\alpha}-(\underline{K}(b, \tilde{\rho}))^{\alpha}\right)+\alpha \underline{C}\left((\underline{K}(g, \tilde{\rho}))^{\alpha-1}-(\underline{K}(b, \tilde{\rho}))^{\alpha-1}\right)\right)\right]=0 .
\end{aligned}
$$

On the other hand, writing the equality of returns to investors for countries declaring Bad states in both groups, i.e., considering the right-hand side of (3), we see that

$$
\alpha A(b)(\underline{K}(b, \tilde{\rho}))^{\alpha-1}=\alpha A(b)(\bar{K}(b, \tilde{\rho}))^{\alpha-1}, \quad \text { that is, } \underline{K}(b, \tilde{\rho})=\bar{K}(b, \tilde{\rho}) .
$$

Hence,

$$
\begin{aligned}
& \mathbb{E}\left[A(b)\left((1-\alpha)(\bar{K}(g, \tilde{\rho}))^{\alpha}+\alpha \bar{C}\left((\bar{K}(g, \tilde{\rho}))^{\alpha-1}-(\bar{K}(b, \tilde{\rho}))^{\alpha-1}\right)\right)\right. \\
& \left.\quad-A(b)\left((1-\alpha)(\underline{K}(g, \tilde{\rho}))^{\alpha}+\alpha \underline{C}\left((\underline{K}(g, \tilde{\rho}))^{\alpha-1}-(\underline{K}(b, \tilde{\rho}))^{\alpha-1}\right)\right)\right]=0 .
\end{aligned}
$$

In particular, substituting the lower bound $\underline{C}<\bar{C}$ and using $\underline{K}(g, \tilde{\rho})>\underline{K}(b, \tilde{\rho})$, which entails the inequality $-(\underline{K}(g, \tilde{\rho}))^{\alpha-1}+(\underline{K}(b, \tilde{\rho}))^{\alpha-1}<0$, we get

$$
\begin{aligned}
& \mathbb{E}\left[A(b)\left((1-\alpha)(\bar{K}(g, \tilde{\rho}))^{\alpha}+\alpha \bar{C}\left((\bar{K}(g, \tilde{\rho}))^{\alpha-1}-(\bar{K}(b, \tilde{\rho}))^{\alpha-1}\right)\right)\right. \\
& \left.\quad-A(b)\left((1-\alpha)(\underline{K}(g, \tilde{\rho}))^{\alpha}+\alpha \bar{C}\left((\underline{K}(g, \tilde{\rho}))^{\alpha-1}-(\underline{K}(b, \tilde{\rho}))^{\alpha-1}\right)\right)\right]>0,
\end{aligned}
$$

which indicates that (6) is satisfied with the function $\psi_{\alpha, \bar{C}}(x)=A(b)\left((1-\alpha) x^{\alpha}+\alpha \bar{C} x^{\alpha-1}\right)$. The latter function is increasing on $[\bar{C},+\infty[$ (as can be seen by computing its derivative); if $\underline{K}(b, \tilde{\rho}) \geqslant \bar{C}$ for all $\hat{\rho}$, then (6) is satisfied with an increasing function and the proof is concluded. Otherwise, there exists a $\hat{\rho}$ such that $\underline{K}(b, \tilde{\rho})<\bar{C} \leqslant \bar{K}(b, \tilde{\rho})$ and the desired necessary and sufficient condition for $\bar{\varphi}<\underline{\varphi}$ 
is met as well.

Proof of Proposition 4. We consider two groups of countries with barriers to capital flows of $\tau<1$ and 1 respectively, with $\tau<1$ being the fraction of returns that can be recuperated by investors expost; quantities related to countries in the group with capital flow barriers $\tau$ will be indexed by the overline bar. We need to prove that $\bar{\varphi}<\varphi$ : in equilibrium, when suffering a Bad state of the economy, the countries with no barriers to flows misreport more often than the countries with barriers $\tau$.

The ex-ante equality of returns demanded by investors (3) for countries declaring Bad states in both groups is now

$$
\begin{aligned}
\tau \alpha A(b)(\bar{K}(b, \tilde{\rho}))^{\alpha-1} & =\tau \alpha\left(\frac{\hat{\rho}}{\hat{\rho}+\bar{\varphi}(1-\hat{\rho})} A(g)+\frac{\bar{\varphi}(1-\hat{\rho})}{\hat{\rho}+\bar{\varphi}(1-\hat{\rho})} A(b)\right)(\bar{K}(g, \tilde{\rho}))^{\alpha-1} \\
= & \alpha A(b)(\underline{K}(b, \tilde{\rho}))^{\alpha-1}=\alpha\left(\frac{\hat{\rho}}{\hat{\rho}+\underline{\varphi}(1-\hat{\rho})} A(g)+\frac{\underline{\varphi}(1-\hat{\rho})}{\hat{\rho}+\underline{\varphi}(1-\hat{\rho})} A(b)\right)(\underline{K}(g, \tilde{\rho}))^{\alpha-1} ;
\end{aligned}
$$

that is, $\bar{K}(b, \tilde{\rho})=\tau^{1 /(1-\alpha)} \underline{K}(b, \tilde{\rho})<\underline{K}(b, \tilde{\rho})$ and, as in (5), we set $\bar{K}(b, \tilde{\rho})=\Xi(\hat{\rho}, \bar{\varphi}) \bar{K}(g, \tilde{\rho})$ and $\underline{K}(b, \tilde{\rho})=\Xi(\hat{\rho}, \underline{\varphi}) \underline{K}(g, \tilde{\rho})$. Note that the closed-form expression of $\Xi$ indicates that $\varphi \mapsto \Xi(\hat{\rho}, \varphi)$ is strictly increasing, for all $\hat{\rho}$. We also introduce the factors $\lambda(b, \hat{\rho})$ and $\lambda(g, \hat{\rho})$ such that

$$
\bar{K}(b, \tilde{\rho})=\lambda(b, \hat{\rho}) \underline{K}(b, \tilde{\rho}) \quad \text { and } \quad \bar{K}(g, \tilde{\rho})=\lambda(g, \hat{\rho}) \underline{K}(g, \tilde{\rho}) .
$$

Combining all these notations, we see that

$$
\lambda(b, \hat{\rho}) \Xi(\hat{\rho}, \underline{\varphi}) \underline{K}(g, \tilde{\rho})=\lambda(b, \hat{\rho}) \underline{K}(b, \tilde{\rho})=\bar{K}(b, \tilde{\rho})=\Xi(\hat{\rho}, \bar{\varphi}) \bar{K}(g, \tilde{\rho})=\Xi(\hat{\rho}, \bar{\varphi}) \lambda(g, \hat{\rho}) \underline{K}(g, \tilde{\rho}),
$$

thus,

$$
\lambda(b, \hat{\rho}) \Xi(\hat{\rho}, \underline{\varphi})=\Xi(\hat{\rho}, \bar{\varphi}) \lambda(g, \hat{\rho}) .
$$

In view of this inequality, since $\Xi$ is increasing in its second argument, to show the desired inequality $\bar{\varphi}<\underline{\varphi}$, it suffices to show that $\lambda(b, \hat{\rho})<\lambda(g, \hat{\rho})$ for at least one ${ }^{49}$ realization $\hat{\rho}$. Since we showed above that $\lambda(b, \hat{\rho})=\tau^{1 /(1-\alpha)}<1$, we can assume that $\lambda(g, \hat{\rho})<1$ for all $\hat{\rho}$ (otherwise, the needed property is immediately seen to hold).

Introducing the function $\psi_{\alpha, C}(x)=A(b)\left((1-\alpha) x^{\alpha}+\alpha C x^{\alpha-1}\right)$, the equilibrium condition (7) with respect to the incentives of countries can be rewritten in this case as

$$
\begin{aligned}
& \mathbb{E}\left[\psi_{\alpha, C}(\bar{K}(g, \tilde{\rho}))-\psi_{\alpha, C}(\bar{K}(b, \tilde{\rho}))-\psi_{\alpha, C}(\underline{K}(g, \tilde{\rho}))+\psi_{\alpha, C}(\underline{K}(b, \tilde{\rho}))\right] \\
& \quad=\mathbb{E}\left[\psi_{\alpha, C}(\lambda(g, \hat{\rho}) \underline{K}(g, \tilde{\rho}))-\psi_{\alpha, C}(\underline{K}(g, \tilde{\rho}))-\psi_{\alpha, C}(\lambda(b, \hat{\rho}) \underline{K}(b, \tilde{\rho}))+\psi_{\alpha, C}(\underline{K}(b, \tilde{\rho}))\right]=0 ;
\end{aligned}
$$

\footnotetext{
${ }^{49}$ It can be shown that it will then hold for all realizations of $\hat{\rho}$.
} 
or, introducing the function of two variables $\Psi_{\alpha, C}(x, t)=\psi_{\alpha, C}(t x)-\psi_{\alpha, C}(x)$,

$$
\mathbb{E}\left[\Psi_{\alpha, C}(\underline{K}(g, \tilde{\rho}), \lambda(g, \hat{\rho}))\right]=\mathbb{E}\left[\Psi_{\alpha, C}(\underline{K}(b, \tilde{\rho}), \lambda(b, \hat{\rho}))\right] .
$$

We have that $\underline{K}(b, \tilde{\rho})<\underline{K}(g, \tilde{\rho})$ as proceeds from (8) and will show below that $\Psi_{\alpha, C}$ is decreasing in its first argument and increasing in its second argument; in view of the equality (9) this will entail that $\lambda(g, \hat{\rho})>\lambda(b, \hat{\rho})$ for some $\hat{\rho}$ and will conclude the proof.

Recall that we indicated above that $\lambda(g, \hat{\rho})<1$ and $\lambda(b, \hat{\rho})<1$ for all $\hat{\rho}$. The fact that the mapping $x \in\left[C,+\infty\left[\mapsto \Psi_{\alpha, C}(x, t)\right.\right.$ is decreasing for all $t<1$ comes directly from its closed-form expression,

$$
\Psi_{\alpha, C}(x, t)=A(b)((1-\alpha) \underbrace{\left(t^{\alpha}-1\right)}_{<0} \underbrace{x^{\alpha}}_{\text {increasing }}+\alpha C \underbrace{\left(t^{\alpha-1}-1\right)}_{>0} \underbrace{x^{\alpha-1}}_{\text {decreasing }}) .
$$

To study the monotonicity in $t$, we note that $t \mapsto \Psi_{\alpha, C}(x, t)$ has the monotonicity of $t \mapsto \psi_{\alpha, C}(t x)$. But we already indicated at the end of the proof of Proposition 3 that $\psi_{\alpha, C}$ was increasing on $[C,+\infty[$; since we only consider here pairs $(x, t)$ such that $t x \geqslant C$ (these pairs are such that $t x=\bar{K}(b, \tilde{\rho})$ or $\bar{K}(g, \tilde{\rho}))$, this show that $t \mapsto \Psi_{\alpha, C}(x, t)$ is indeed increasing for all $x$ of interest, as claimed above.

For two groups of countries with barriers to capital flows of $\underline{\tau}<1$ and $\bar{\tau}<1$ respectively, where $\underline{\tau}<$ $\bar{\tau}<1$ the above proof can be easily restated with rescaling the barriers to $\tau \equiv \underline{\tau} / \bar{\tau}$ for the country with lower barriers. Neither the relative relationships between capital levels invested in these economies nor the country payoffs (and the equilibrium condition) depend directly on these barriers.

\section{C.2 Complements to Section 5: The balance of payments data and Benford's law}

\section{C.2.1 On property $\mathbf{P 1}$}

We provide below a more detailed presentation of its mathematical foundations, with references to the mathematical literature, and some other applications to macroeconomic data.

Deterministic sequences. The simplest mathematical generating model is the following; it is used as a keystone to construct the subsequent models of this paragraph and of the models discussed in Section 5.1.1. Pick numbers $Y_{1}, \ldots, Y_{n}$ at random in the interval $[1,10)$ such that the random variables $\log _{10} Y_{1}, \ldots, \log _{10} Y_{n}$ are uniformly distributed over the interval [0,1); that is, we consider $\log _{-}$ uniform mantissas. Then, for all natural integers $k_{1}, \ldots, k_{n}$, the distribution of the first digits of the numbers $Y_{1} \times 10^{k_{1}}, \ldots, Y_{n} \times 10^{k_{n}}$ converges to Benford's law as $n$ grows.

Based on this, Diaconis (1977) shows that geometric sequences $\left\{a, a^{2}, \ldots, a^{n}\right\}$ with ratio $a$ such that $\log _{10} a$ is irrational ${ }^{50}$ lead to sets of data that conform more and more closely to Benford's law as

\footnotetext{
${ }^{50}$ Otherwise the sequence of the first digits is cyclic and has asymptotic proportions that are rational and thus are
} 
$n$ grows. That is, almost all geometric sequences lead to Benford's law as the set of rational numbers has a null measure within the set of all real numbers. This implies that in practice all geometric sequences linked to real data lead the data to obey Benford's law.

A natural and important extension of this result is that for differences of such geometric processes the Benford's law is obeyed. Lemma 2.4 in Kaynar et al. (2010) shows that for given ratios $a$ and $b$ (such that $\log _{10} a$ is irrational) and any real numbers $\alpha$ and $\beta$, the sets

$$
\left\{\alpha a-\beta b, \alpha a^{2}-\beta b^{2}, \ldots, \alpha a^{n}-\beta b^{n}\right\}
$$

conform more and more closely to Benford's law as $n$ grows.

Extensions to certain random geometric sequences. A combination of the results of Holewijn (1969) and of some other classical results recalled, e.g., in Diaconis (1977) indicates that when $\left(X_{j}\right)$ is a sequence of independent and identically distributed random variables, the sets

$$
\left\{X_{1}, X_{1} X_{2}, \ldots, X_{1} X_{2} \cdots X_{n}\right\}
$$

also conform more and more closely to Benford's law as $n$ grows provided that the common characteristic function $\varphi$ of the $X_{j}$ is such that $\psi(2 \pi k) \neq 1$ for all natural integers $k$; this condition is satisfied for almost all distributions. This result extends to differences of processes of the form (11), in the same vein as in (10).

Many other extensions and variations around them exist (such as the considerations of martingale difference sequences, which relaxes the hypotheses of independence and/or identical distribution), which we do not mention in detail. We only indicate a recent fundamental article of Schürger (2008), who studies the conformity to Benford's law of processes of the form

$$
Z_{t}=Z_{0} \exp \left(\mu t+\sum_{s=1}^{t}\left(X_{s}-V_{s} / 2\right)\right), \quad t=1,2, \ldots
$$

where $\left(X_{t}\right)$ is a martingale difference sequence with associated conditional variances $\left(V_{t}\right)$; this process is indeed of the form (11).

Other applications to macroeconomic data We indicated in Section 5.1.1 that various extensions could be instantiated as special cases of the multiplicative form discussed above, including the case of structural breaks that could be associated with deep economic crises or large shocks and/or the presence of some errors or misreports in the data.

different from the ones of Benford's law; consider the simple example when $\log _{10} a=2 / 3$, in which case the sequence is $10^{2 k / 3}$ and contains only elements with first digits equal to the first digits of $10^{2 / 3}, 10^{4 / 3}$, and 100 , that is, $4,2,1$; the asymptotic repartition of the first digits is uniform between these three numbers. 
Indeed, as far as the structural breaks are concerned, we suppose that at each round, a break can occur independently from the past, with a constant (small) probability. In this case, the $X_{j}$ are given by a two-step randomization, the first step determining whether a break occurs and the second one providing the adequate multiplicative factor, drawn at random according to a distribution depending on the regime. Of course, for the conformity of the sets (11) to hold approximatively, we will then need even larger values of $n$.

Moreover, the same two-step modeling shows that the presence of some errors or misreports in the data does not impact per se its conformity to Benford's law, provided that these errors can be described in some random multiplicative manner.

\section{C.2.2 More on persistence issues}

We report here the perhaps deceiving results that are obtained when testing whether the data corresponding to each country is Benford distributed or not: the test accepts the hypothesis of conformity to Benford's law at the level $5 \%$ for 58 countries but rejects it for 44 other countries (whereas no conclusion can be drawn for the remaining country, Serbia, for which fewer than $N=110$ observations are available). That is, if one chooses one country with enough observations at random, the obtained data set will not conform to Benford's law with probability $44 /(44+58) \approx 43.1 \%$. There is no striking pattern in countries for which we obtain these rejections. For example, we reject the null hypothesis for the USA, France, Switzerland, Japan or Canada while for many non-OECD countries we cannot. Some examples of obtained empirical distributions for different countries are reported in Figure 1. Out of the individual three series presented (United Kingdom, Colombia and Zimbabwe) only for the series for United Kingdom (976 observations) the hypothesis that they are drawn from a Benford's distribution are not rejected at a $1 \%$ level. The Colombian series (564 observations), although with sizeable discrepancies, still has a distribution visually similar to Benford's law; the two first digits are also more common in the data from Zimbabwe (288 observations).

On many levels it is unsurprising that many individual series with few observations will not adhere to Benford's law (for example see Gonzalez-Garcia and Pastor [2009]). As already noted and illustrated by simulations in Nye and Moul (2007, Section I), it seems necessary to consider several countries for the corresponding data subset to conform to Benford's law; this is to increase heterogeneity at the initial levels and match the requirement provided by the property $\mathrm{P} 2$ that the number $n$ of separately drawn subsets should be large. For example, although for many individual OECD countries the distribution of the first digits does not conform to Benford's law (as noted above), for the entire set of the OECD countries for which we have data (which includes 22 countries) Benford's distribution cannot be rejected (see Table III). 


\section{C.2.3 Another way for correcting for persistence issues: selecting some series from the balance of payments data}

All methods described in the main body of this paper relied on considering for each country-quarter pair all the 13 series that occur in the balance of payments data we obtained from the IMF. We now study what happens when only some series are selected. To that end we considered two choices, the independent series and the less persistent series.

The first subset of series is formed by taking into account the identities that occur in the balance of payments data and by removing a series for each such identity; several choices were possible and the one we made is the following: Current account; Goods; Services; Income; Financial account; Direct investment; Portfolio investment; Financial derivatives; Reserve assets; Net errors and omissions. That is, we dropped the series: Current transfers; Capital account; Other investment.

The second subset of series is formed by taking, out of the 13 series, the 6 series which showed on average the smallest persistence from one quarter to another. For each series and each country, we computed the number of breaks in the sequence of the first digits indexed by quarters and then considered the average of these results with respect to countries. The series included in the resulting subset are then: Financial account; Portfolio investment; Other investment; Financial derivatives; Reserve assets; Net errors and omissions.

We first study what happens for individual countries. We recall that using all series, the data corresponding to 58 countries could be said to conform to Benford's law whereas for 44 countries, it did not pass the test (whereas for 1 country there was not enough data). For independent series, the respective figures are 54, 44, and 5 while for the less persistent series, we obtain 78, 15, and 10. Clearly, the persistence of the series does matter for rejections of Benford's distribution for individual countries.

In addition to this comparison, we also used the same random drawing methodology as above to obtain Table XV. We only reported therein the results for all series and the less persistent ones since the consideration of the independent series instead of all series almost does not change the picture for any pair $(C, M)$ (detailed results provided upon request). On the other hand, the restriction to series chosen as being the less persistent ones ensures that for almost all pairs, the rejection rates get close to or smaller than $5 \%$.

This illustrates once again that the observed persistence is due to a lack of independence in the quarter-to-quarter values of the series and is not linked to an intra-quarter dependence caused by the identities between the series of the balance of payments.

Therefore, a method for correcting the persistence issues is to discard the most persistent series in the balance of payments data; the remaining series typically exhibit behavior close to what is predicted by Benford's law (as shown by the small rejection rates indicated in Table XV). The consideration of the less persistent series brings auxiliary information on whether the possible non-conformity to Benford's law may not be due to the persistence of the balance of payments data. However, finding a conformity of the first digits of these less persistent entries with Benford's distribution does not 
indicate that there is no data falsification going on at all.

\section{C.2.4 Associated tables}




\begin{tabular}{l|ccc}
\hline BOP series & Independent series & Less persistent series & All series \\
\hline Current account & yes & no & yes \\
Goods & yes & no & yes \\
Services & yes & no & yes \\
Income & yes & no & yes \\
Current transfers & no & no & yes \\
Capital account & no & no & yes \\
Financial account & yes & yes & yes \\
Direct investment & yes & no & yes \\
Portfolio investment & yes & yes & yes \\
Other investment & no & yes & yes \\
Financial derivatives & yes & yes & yes \\
Reserve assets & yes & yes & yes \\
Net errors and omissions & yes & yes & yes \\
\hline
\end{tabular}

Table XIII: Summary of the three sets of balance of payments series considered in this study. 


\begin{tabular}{ccccc}
\hline $\begin{array}{c}\text { Proportion } \\
f=5 \%\end{array}$ & \multicolumn{5}{c}{ Estimated quantiles (values given in \%) } \\
\hline $\mathrm{N}=2,500$ & $50 \%$ & $95 \%$ & $98 \%$ & $99 \%$ \\
$\mathrm{~N}=5,000$ & 6.2 & 5.7 & 6.2 & 6.5 \\
$\mathrm{~N}=10,000$ & 6.3 & 6.7 & 7.5 & 7.8 \\
$\mathrm{~N}=30,000$ & 6.3 & 6.7 & 7.4 & 7.9 \\
$\mathrm{~N}=50,000$ & 6.2 & 6.7 & 7.2 & 7.6 \\
\hline & & 6.6 & 7.2 & 7.6 \\
\hline $\mathrm{N} \geq 5,000$ & 6.3 & 6.7 & 7.5 & 7.9 \\
\hline
\end{tabular}

\begin{tabular}{ccccc}
\hline $\begin{array}{c}\text { Proportion } \\
f=10 \%\end{array}$ & \multicolumn{5}{c}{ Estimated quantiles (values given in \%) } \\
\hline $\mathrm{N}=1,200$ & $50 \%$ & $95 \%$ & $98 \%$ & $99 \%$ \\
$\mathrm{~N}=2,000$ & 7.0 & 6.3 & 7.2 & 8.0 \\
$\mathrm{~N}=5,000$ & 6.9 & 7.9 & 9.1 & 9.5 \\
$\mathrm{~N}=10,000$ & 6.9 & 7.8 & 9.0 & 9.8 \\
$\mathrm{~N}=30,000$ & 6.8 & 7.5 & 8.7 & 9.9 \\
$\mathrm{~N}=50,000$ & 6.8 & 7.7 & 9.7 & 9.5 \\
\hline & \multicolumn{5}{c}{ Conclusion: upper bounds } \\
\hline $\mathrm{N} \geq 2,000$ & 7.0 & 8.0 & 9.0 & 10.0 \\
\hline
\end{tabular}

\begin{tabular}{ccccc}
\hline $\begin{array}{c}\text { Proportion } \\
f=20 \%\end{array}$ & \multicolumn{5}{c}{ Estimated quantiles (values given in \%) } \\
\hline $\mathrm{N}=1,000$ & 8.7 & $95 \%$ & $98 \%$ & $99 \%$ \\
$\mathrm{~N}=2,000$ & 8.2 & 10.4 & 12.8 & 14.2 \\
$\mathrm{~N}=5,000$ & 8.2 & 10.5 & 13.4 & 14.5 \\
$\mathrm{~N}=10,000$ & 8.5 & 10.2 & 12.6 & 14.3 \\
$\mathrm{~N}=30,000$ & 8.6 & 10.9 & 13.0 & 14.1 \\
$\mathrm{~N}=50,000$ & 8.4 & 9.7 & 11.8 & 13.3 \\
\hline & & Conclusion: upper bounds & \\
\hline $\mathrm{N} \geq 1,000$ & 8.7 & 10.9 & 13.4 & 14.5 \\
\hline
\end{tabular}

Table XIV: Estimates of the quantiles of the distribution of the random variable $\bar{R}_{D, 1000}$ when $D$ is chosen at random according to Benford's law and $f$ equals $5 \%$ (top table), $10 \%$ (middle table), and $20 \%$ (bottom table); estimates were obtained by drawing $D$ at random 1,000 times and computing the associated values of $\bar{R}_{D, 1000}$. 


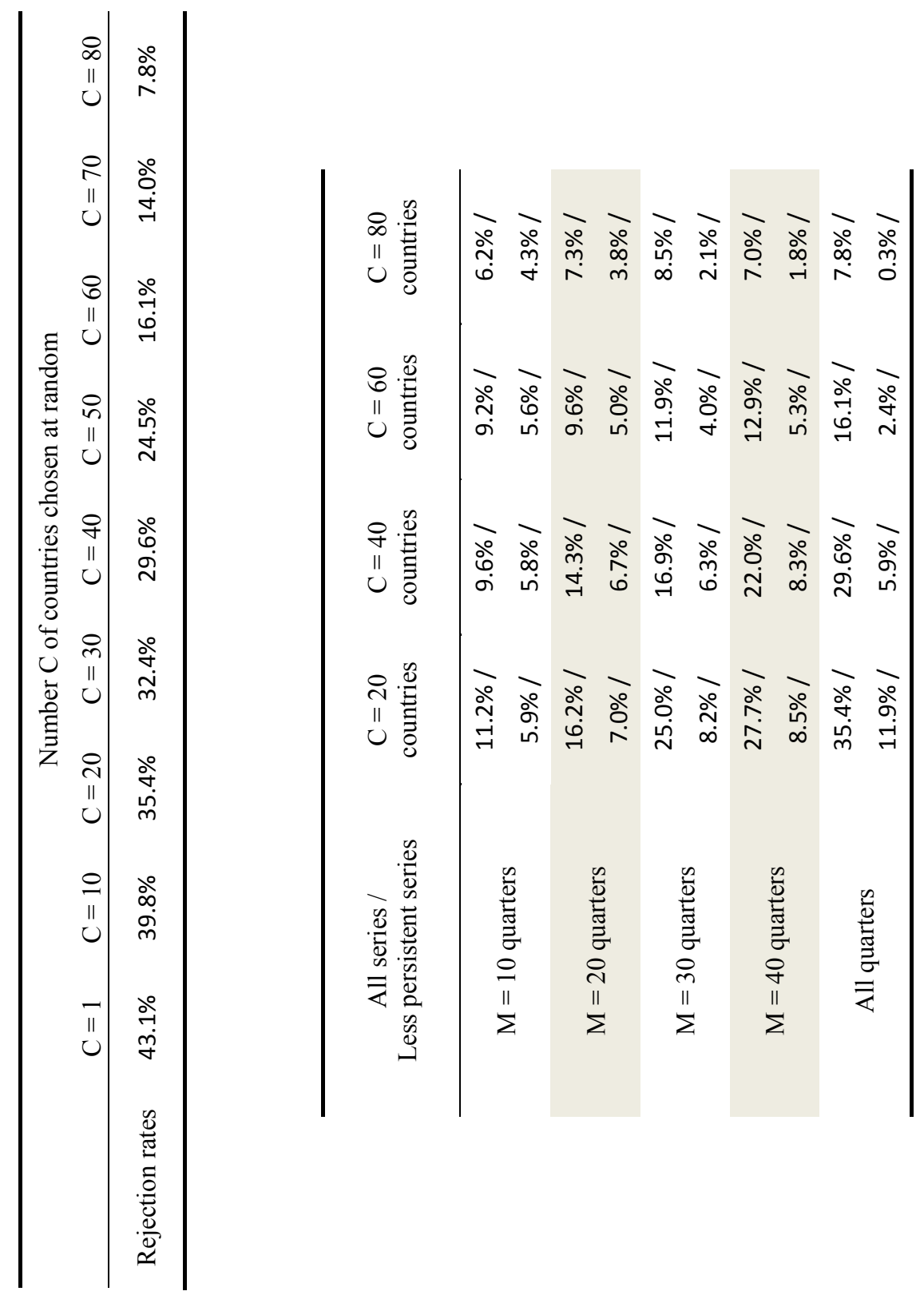

.

$\div \quad 200$

告

के के

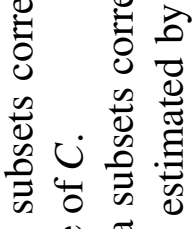

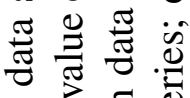

ธี ฮี

总芯芯

$\omega 0$

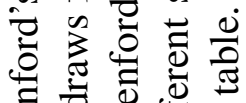

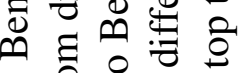

월

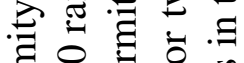

80

号

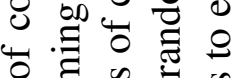

.

仓ै

을을융

焉 䟓

\&

प० 000

ब

ฮ

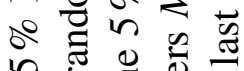

的可导

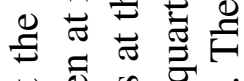

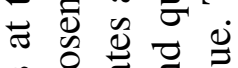

过 흄

जै

욜

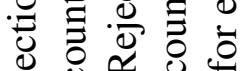

Q

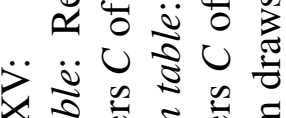

$x$ 㐫

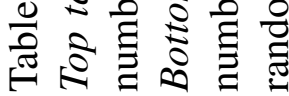




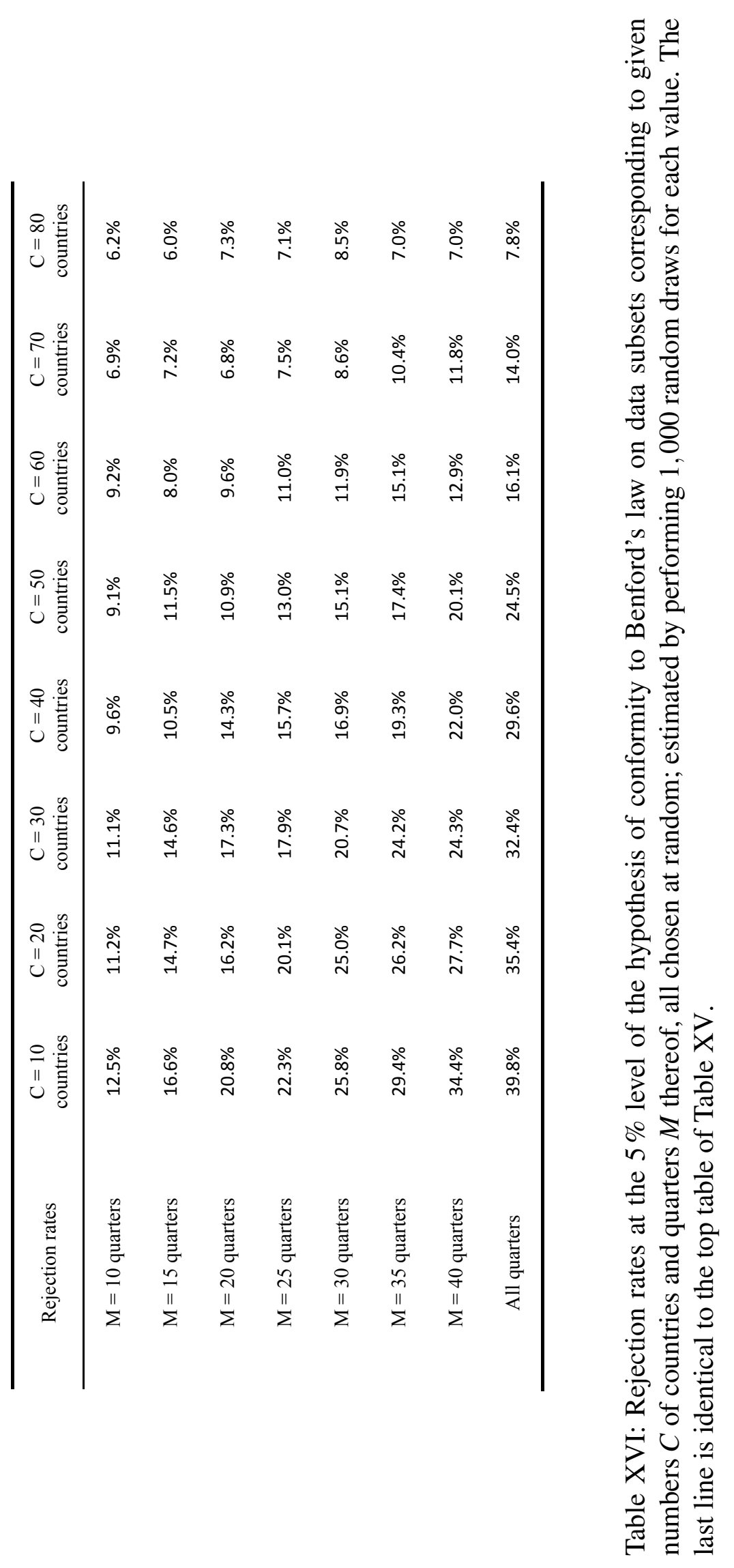




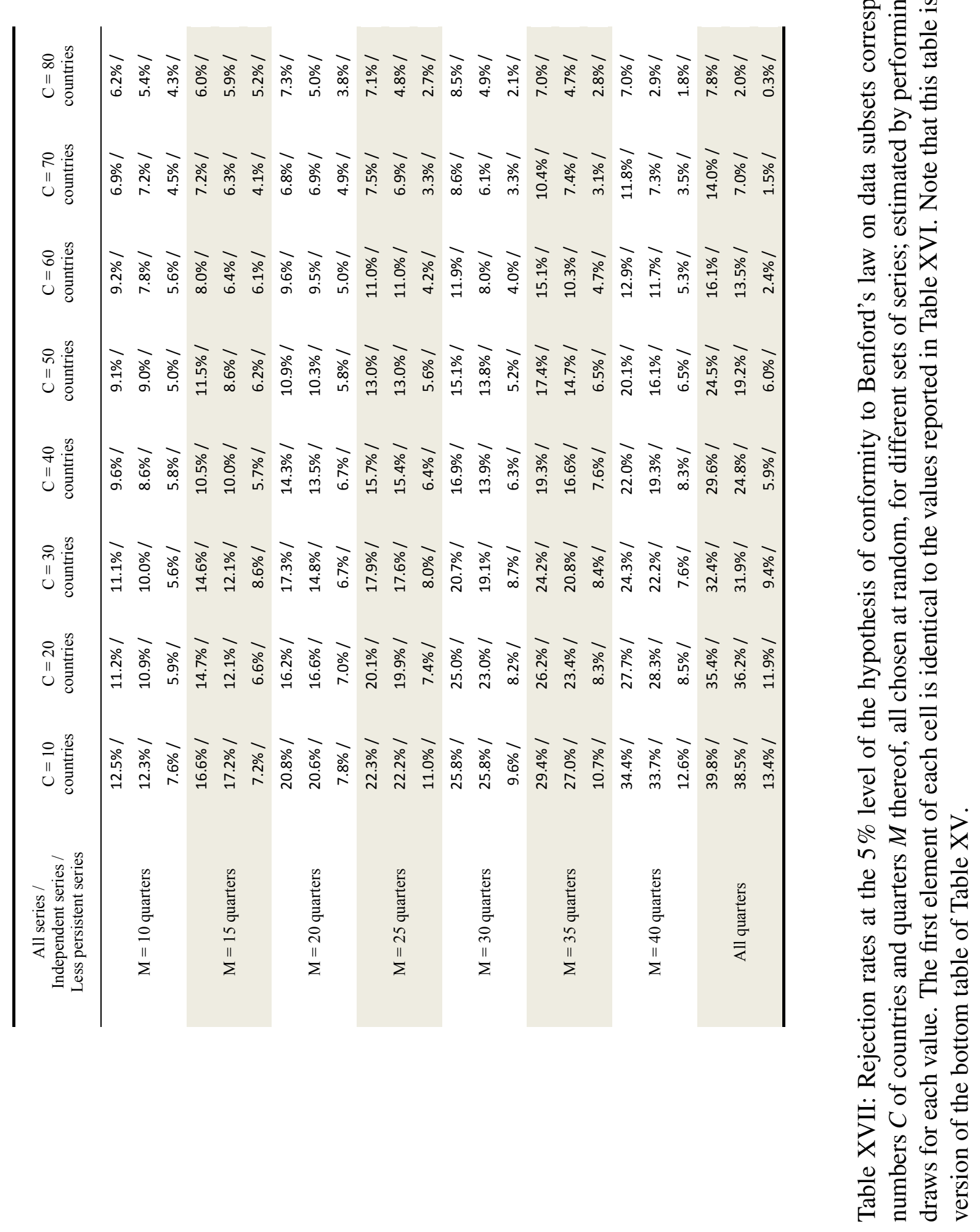




\section{C.3 Countries included in the study}

Albania, Argentina, Armenia, Australia, Austria, Azerbaijan, Bangladesh, Belarus, Belize, Bolivia, Brazil, Bulgaria, Cambodia, Canada, Cape Verde, Chile, Colombia, Costa Rica, Croatia, Cyprus, Czech Republic, Denmark, Ecuador, El Salvador, Eritrea, Estonia, Ethiopia, Finland, France, Georgia, Germany, Greece, Guatemala, Guinea, Honduras, Hungary, Iceland, India, Indonesia, Ireland, Israel, Italy, Japan, Jordan, Kazachstan, Korea, Kirghiz Republic, Laos, Latvia, Lebanon, Lesotho, Lithuania, Macedonia (former Yugoslav Republic of), Madagascar, Malaysia, Malta, Mauritius, Mexico, Moldova, Mongolia, Morocco, Mozambique, Myanmar, Namibia, Nepal, Netherlands, New Zealand, Nicaragua, Nigeria, Norway, Pakistan, Panama, Papua New Guinea, Paraguay, Peru, Philippines, Poland, Portugal, Romania, Russia, Serbia, Singapore, Slovakia, Slovenia, South Africa, Spain, Sri Lanka, Sudan, Suriname, Sweden, Switzerland, Tajikistan, Thailand, Turkey, Turkmenistan, Uganda, Ukraine, United Kingdom, United States, Uruguay, Venezuela, Vietnam, Yemen (Republic of), Zimbabwe

\section{C.4 Complements to Section 6: Results for country-quarter groups based on economic conditions}

\section{C.4.1 Crisis time evidence}

Our model tells us (Proposition 5) that when more than expected fraction of countries experience Bad states of productivity, more countries would misinform investors. Empirically, this is difficult to determine, as we do not know what the expectations of the $\rho$ (the fraction of countries having a Good state) were at any given time point. One can perfectly fathom a period with no global crisis, yet the fraction of the countries with good states of the world being lower than expected, leading to more countries misinforming. Nevertheless, in our sample period, Reinhart and Rogoff (2009) claim that there was one truly unexpected global financial crisis in the years 1997-1998 that originated in Asia and then spread across the globe, and this is what we investigate.

We use the stable sample and yearly data, because we want to have globally roughly the same number of observations for each studied time period. We find some evidence for 1997 but no results for 1998. An interpretation in line with our model is that in 1998 it was already clear that the crisis is going to affect many countries and investors adjusted their $\rho$; so fewer countries misinformed on their state of the world. The evidence for 1997 shows that countries open to capital flows (also those with lower net foreign assets, higher total liabilities and with fixed exchange rates) have rejections of Benford's distribution for their first-digit data at levels lower than a $5 \%$ level. This is what we would expect for our model in Section 3: the benefit of embellishing statistics would be higher when many countries would be in a bad state of the world, and for those that would be open to capital flows. ${ }^{51}$

\footnotetext{
${ }^{51}$ Similar patterns appear for many categories for 1996 and 2006 if we investigate other years; without comprehensive further research it is difficult to determine whether these were years with large unexpected shocks to many countries
} 


\section{C.4.2 Geographical country groups}

For the sake of completeness, and to compare to the previous results of Nye and Moul (2007) or Gonzalez-Garcia and Pastor (2009) who dealt with geographically-based country groupings, we present the goodness-of-fit tests of conformity of the first-digit distributions with Benford's distribution broken down geographically in Table LII (containing both main results and robustness checks). Here we need to rely heavily on the 1-in-10 criterion as the persistence issue may be severe. We find that countries in Africa and the Middle East (grouped together in order to have a sufficient large number of observations) have suspect distributions of the first digits both in the full and the stable samples. Latin American countries in the stable sample, i.e., that provided data at least every year between 1995 and 2007, also have a distribution of first digits for which we can reject the hypothesis that it was drawn from a Benford's distribution. Finally, the 1-in-10 criterion flags the rejection of the null hypothesis at $5 \%$ for the stable sample and at $10 \%$ for the full sample for East European countries. These findings should be taken with caution, however, as there are relatively few countries in each group with many quarters of data each, which may make rejecting the null hypothesis easier (see Section 5.4 for discussion). Tests on the least persistent series do not confirm any of the findings. This may mean that it so happens that African, Middle Eastern and Latin American (and potentially East European) countries have some economic series whose first digits are very persistent (i.e., evolve slowly) when termed in U.S. dollars, and these drive the rejections of Benford's distribution. Furthermore, as there are few countries in each group, there may be little required heterogeneity for the Benford's law to hold in view of property P2; in the goodness-of-fit tests the entire dataseries are taken and mixed together. Wider economic criteria of grouping countries together might therefore be better than narrow geographic ones. It may not be a primary feature of these countries that they provide bad quality data on purpose.

\section{C.4.3 Detailed tables}

The tables included here contain more detailed results for the main results for the stable sample, and the results for the full sample and robustness checks of the findings. Certain tables contain also groupings using some further criteria that may be mentioned in the main text:

- net foreign assets as a ratio of GDP (NFA_GDP);

- liabilities to GDP ratio (LIAB_GDP);

- liabilities excluding foreign direct investment to GDP (LIAB_EXCL_FDI_GDP);

- countries belonging to the OECD (OECD).

across the world that would cause such rejections. There were substantial falls (of over $20 \%$ over some periods) in 2006 global stock market indexes for emerging markets. But this does not indicate how many countries were implicated and whether this would in fact constitute a global and substantial unexpected negative shock. 


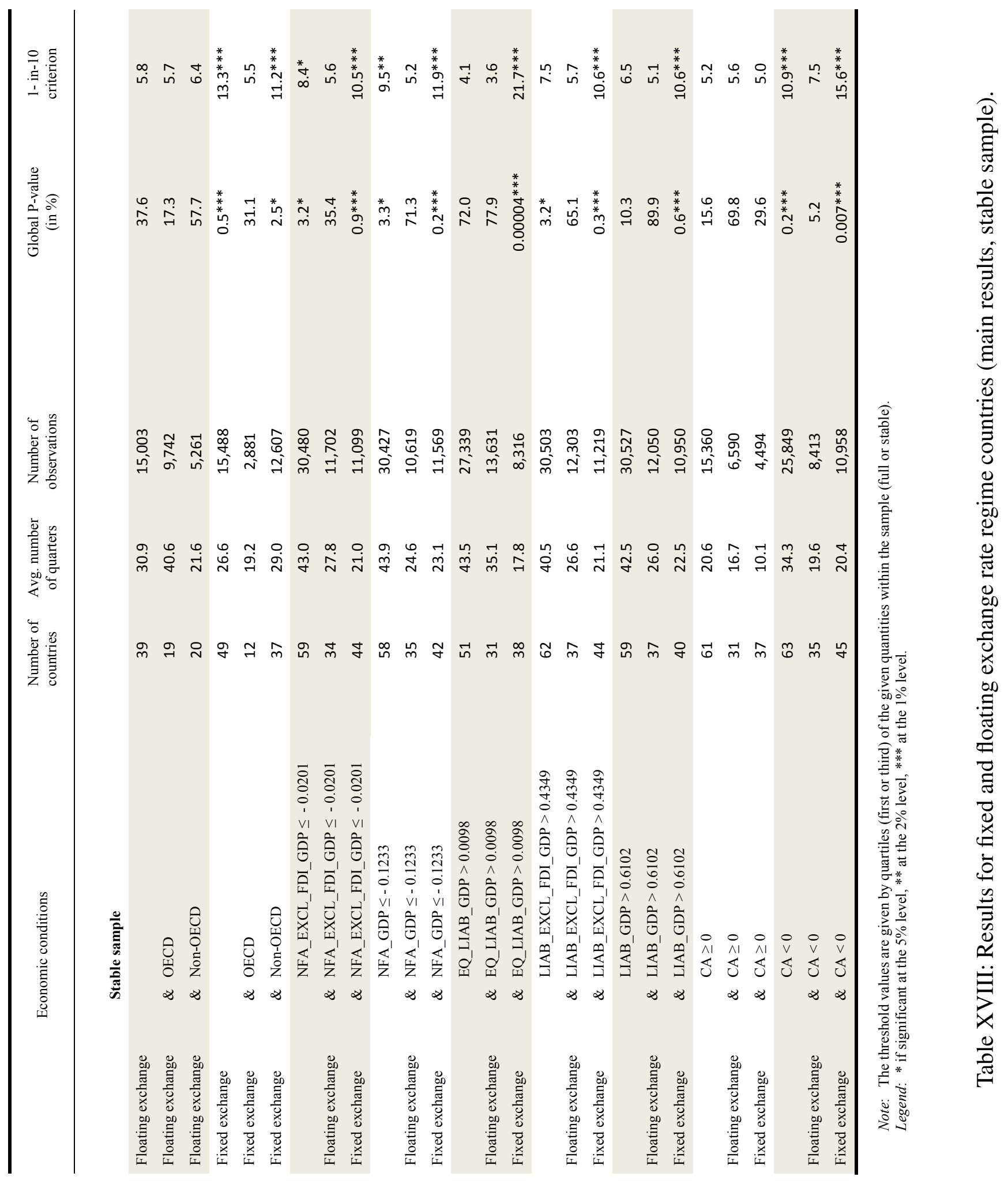




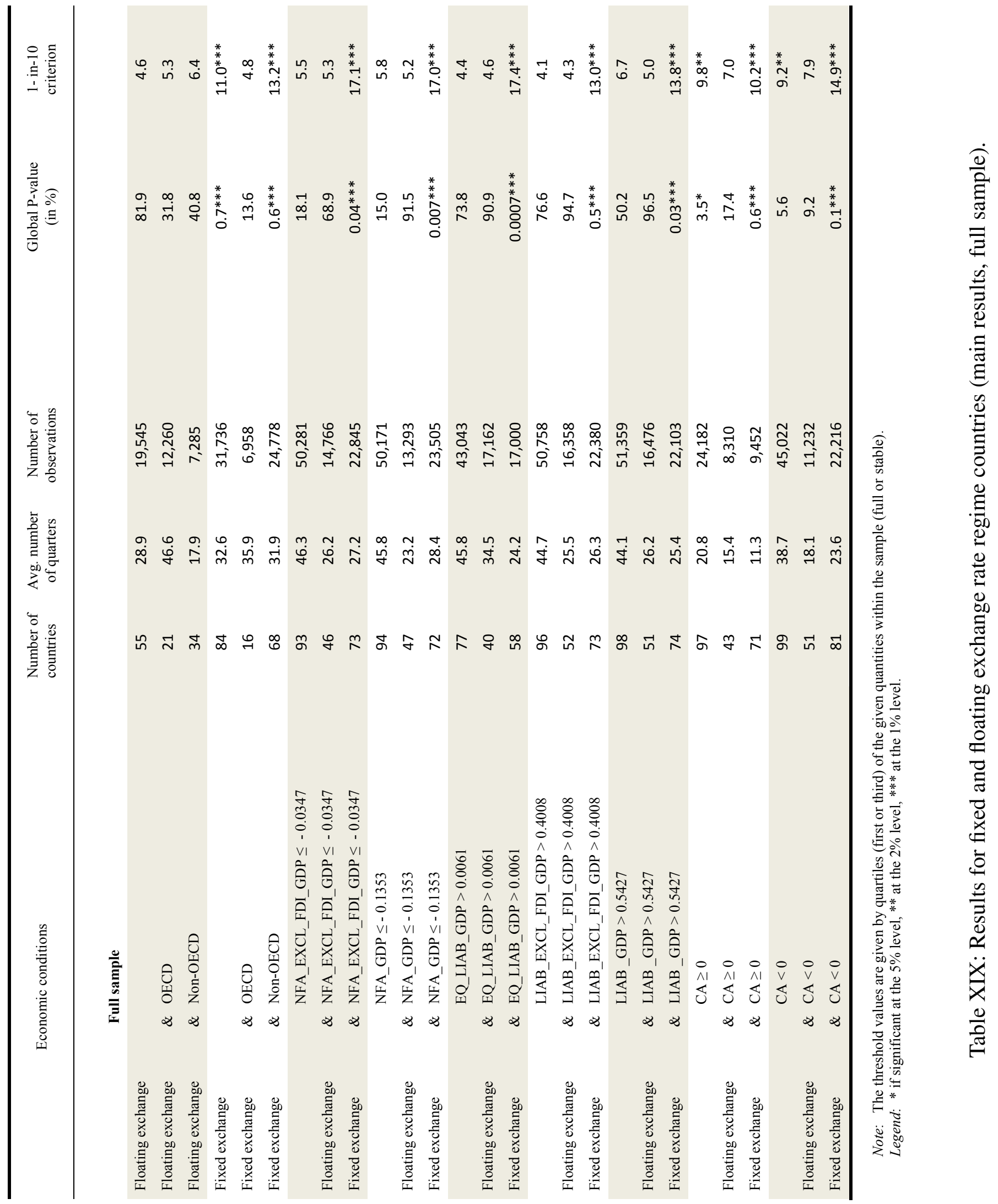




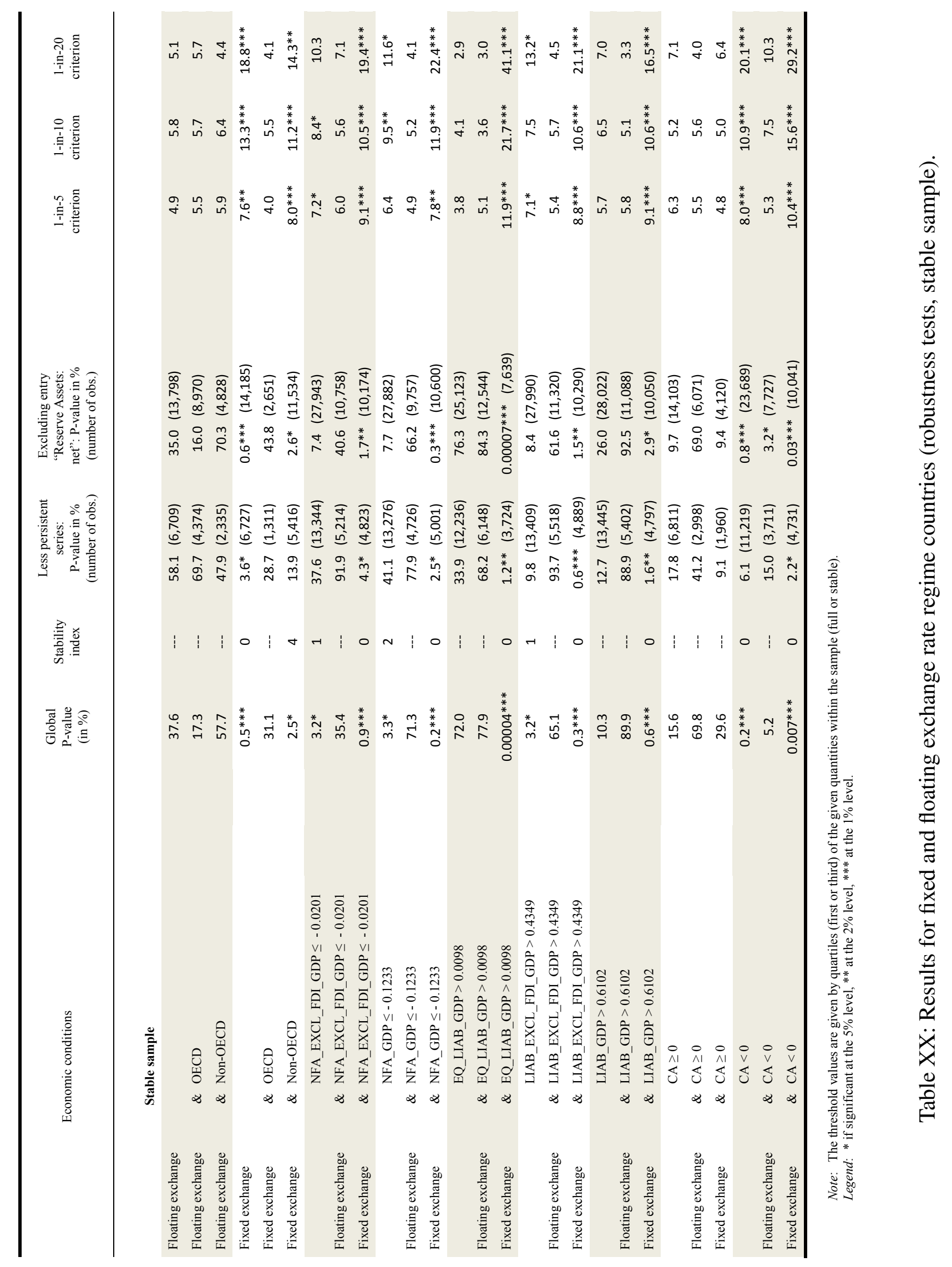




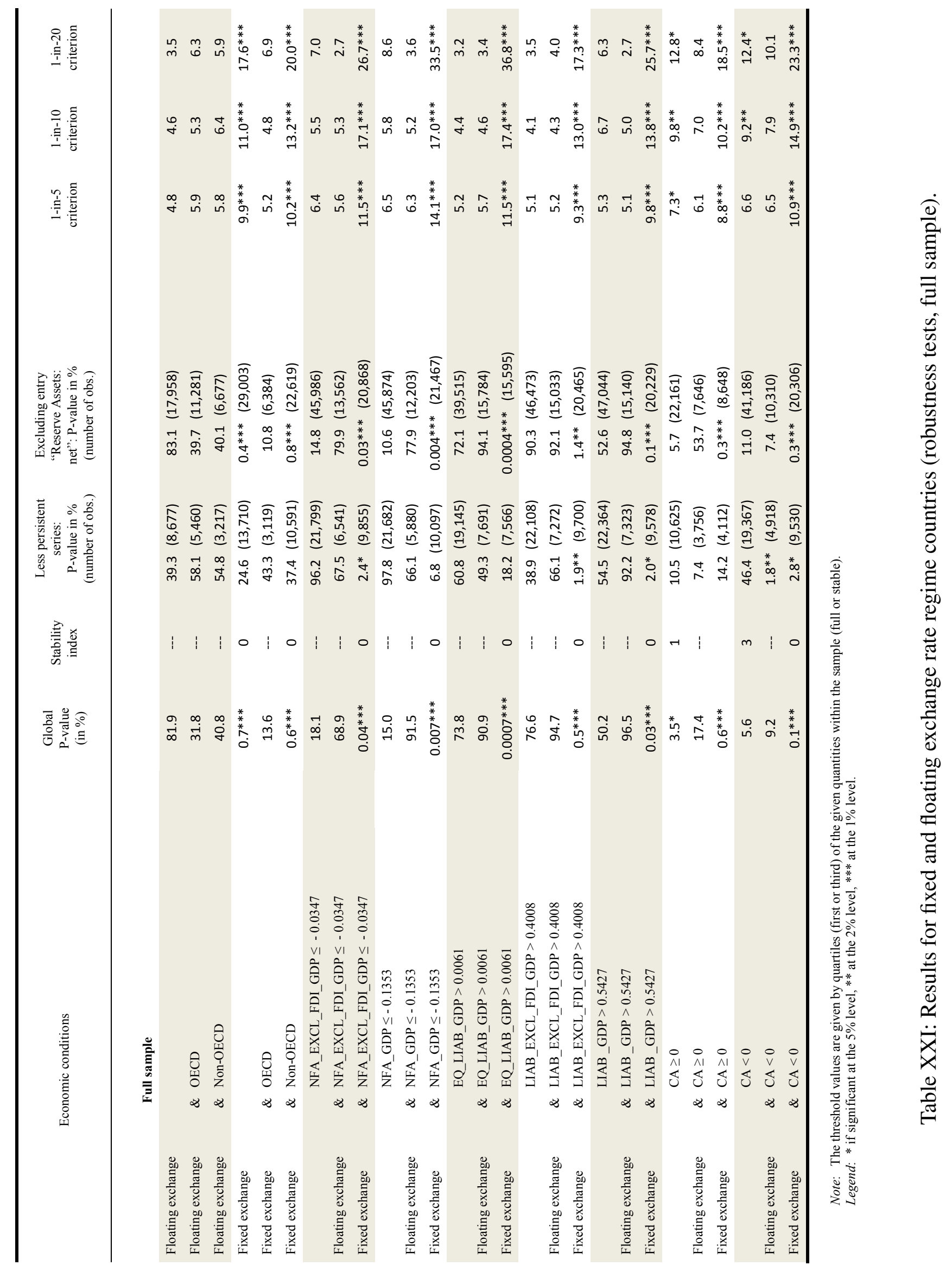




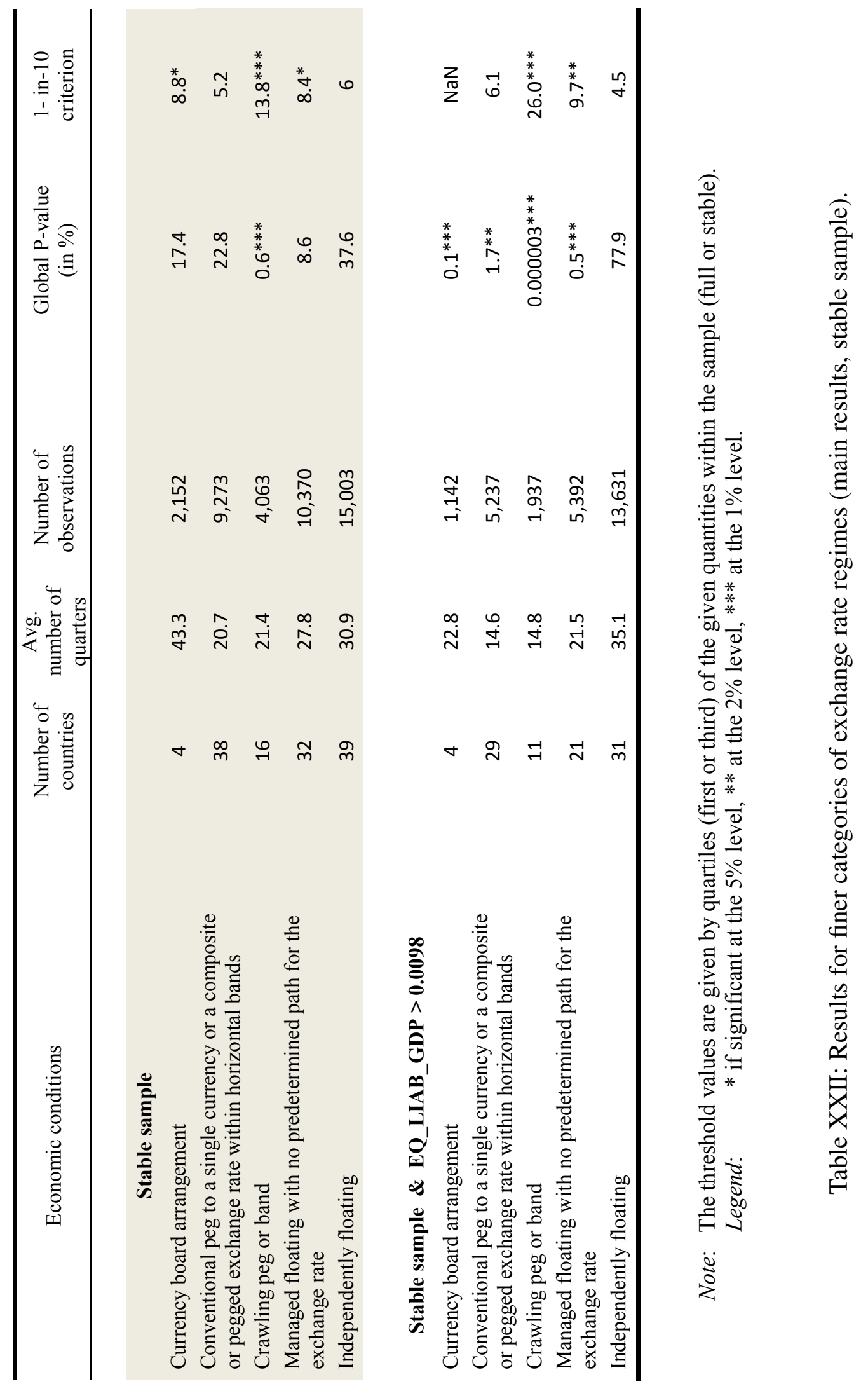




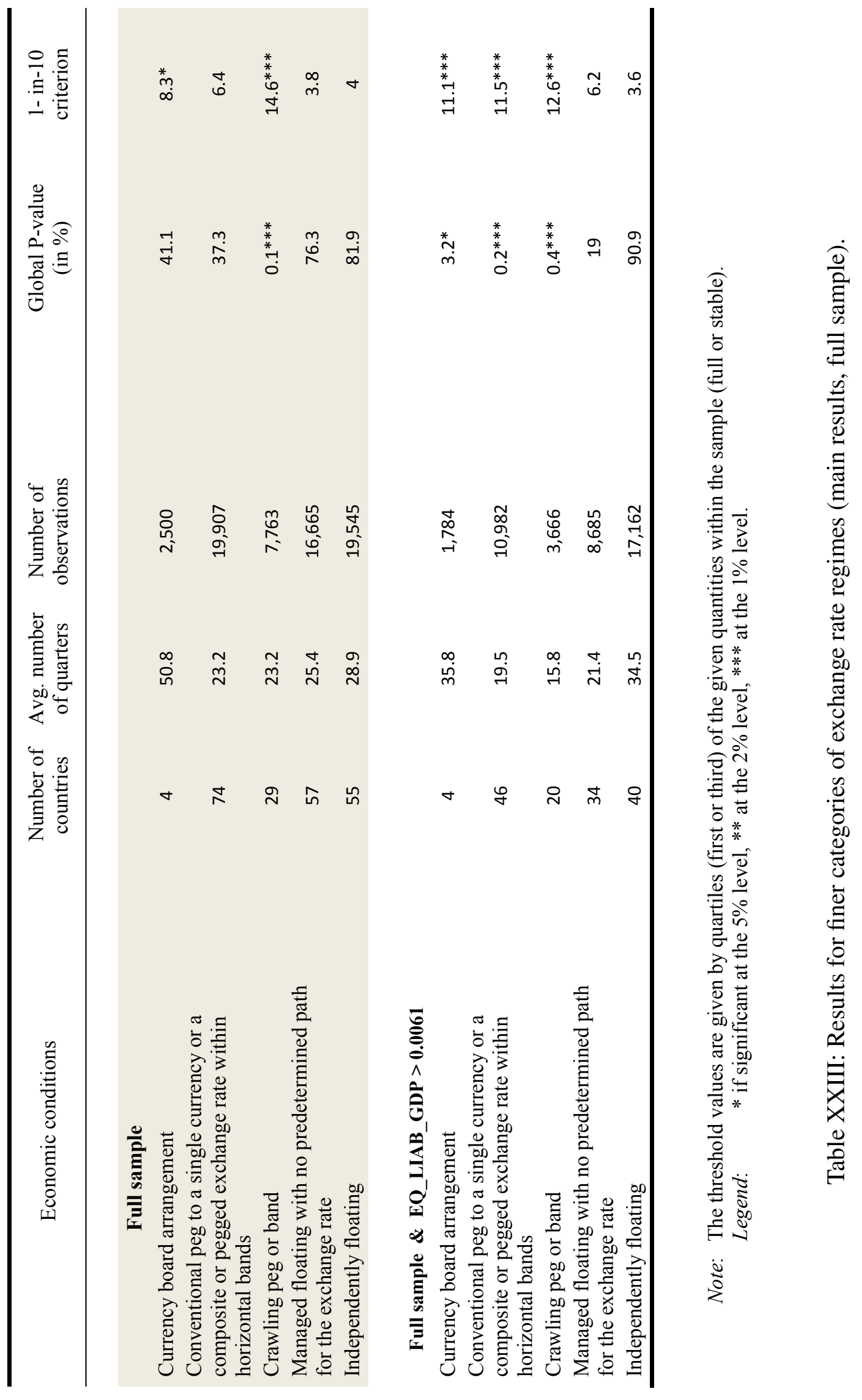




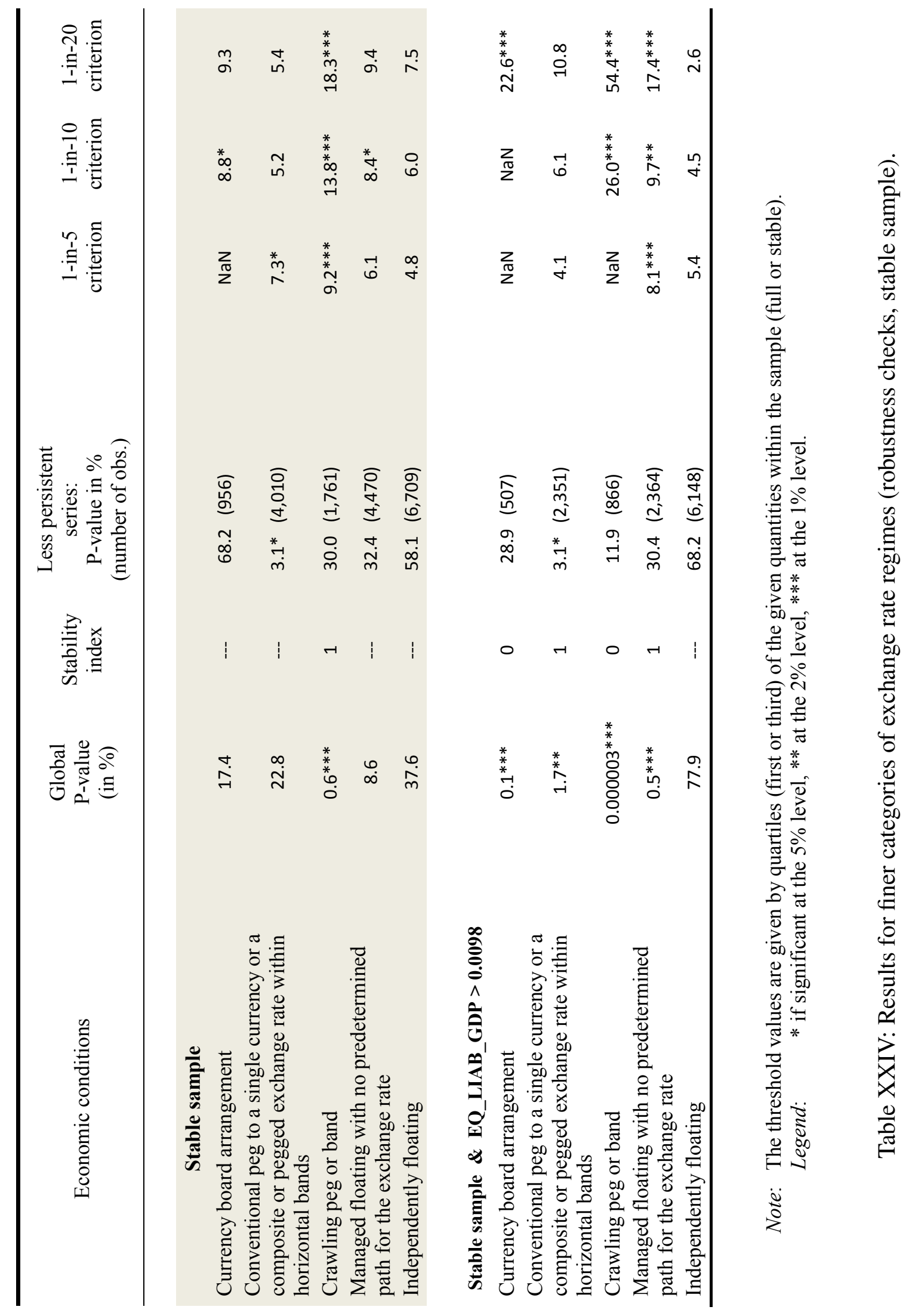




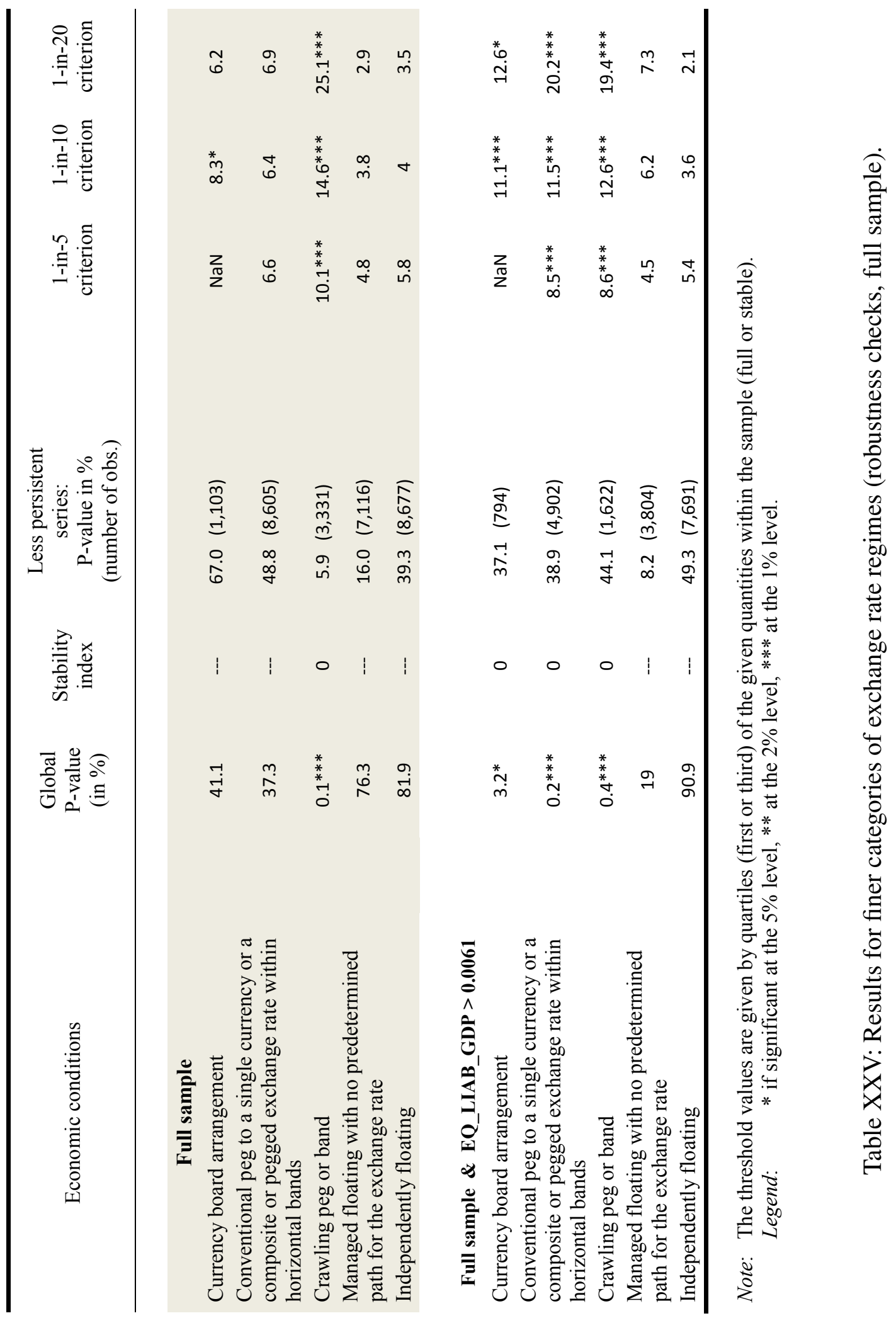




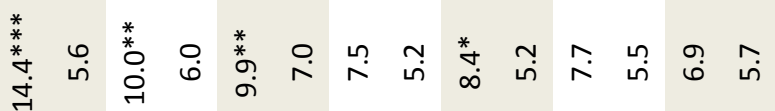

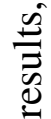

$\frac{\sqrt{2}}{2}$

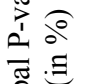

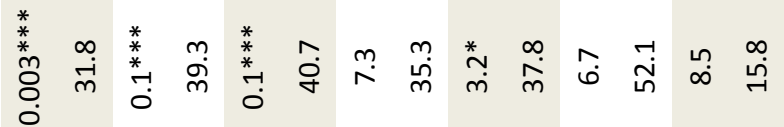

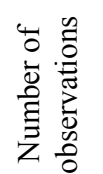

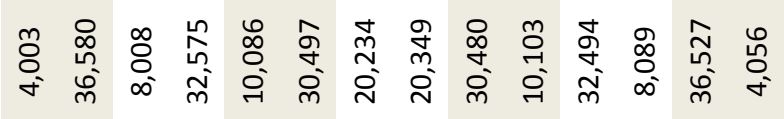

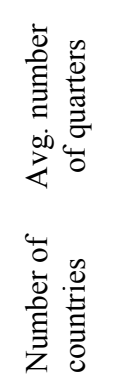

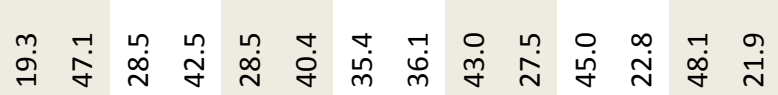

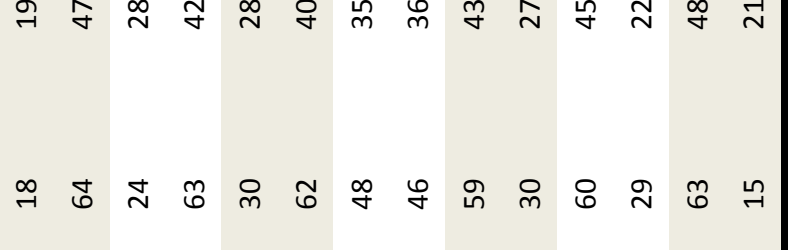

离

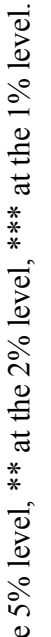

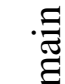

离

苍

อี อี

$\stackrel{\substack{0 \\:}}{:}$

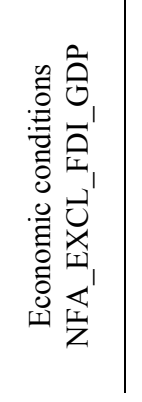

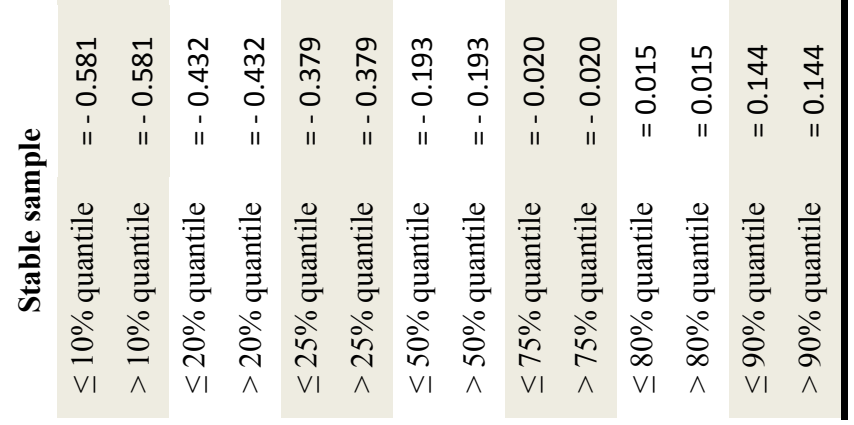

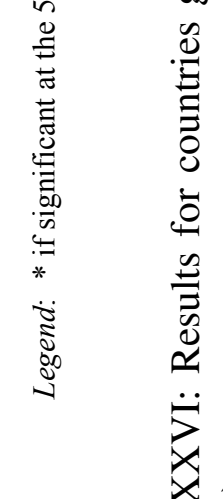

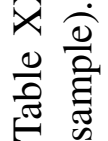




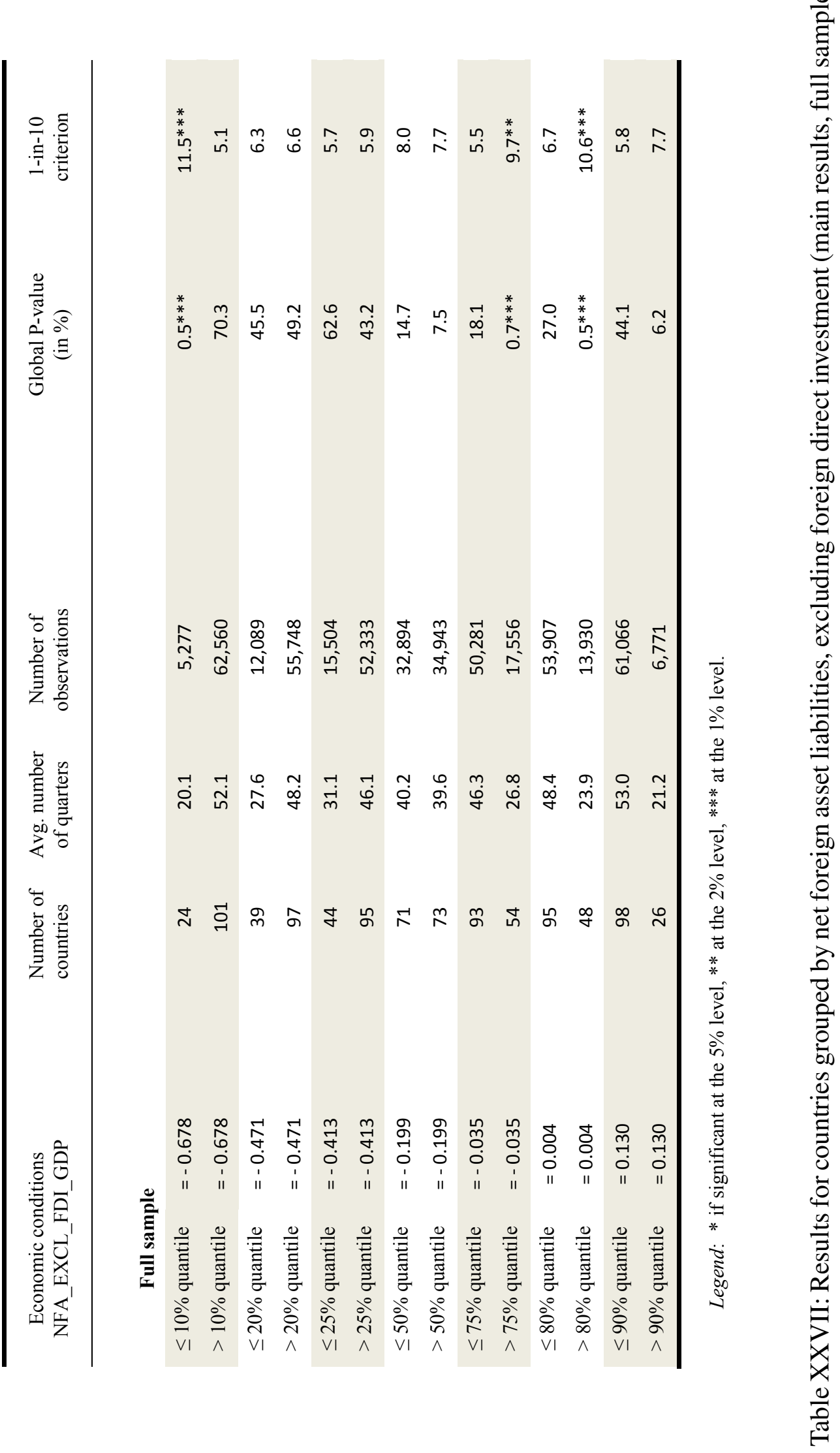




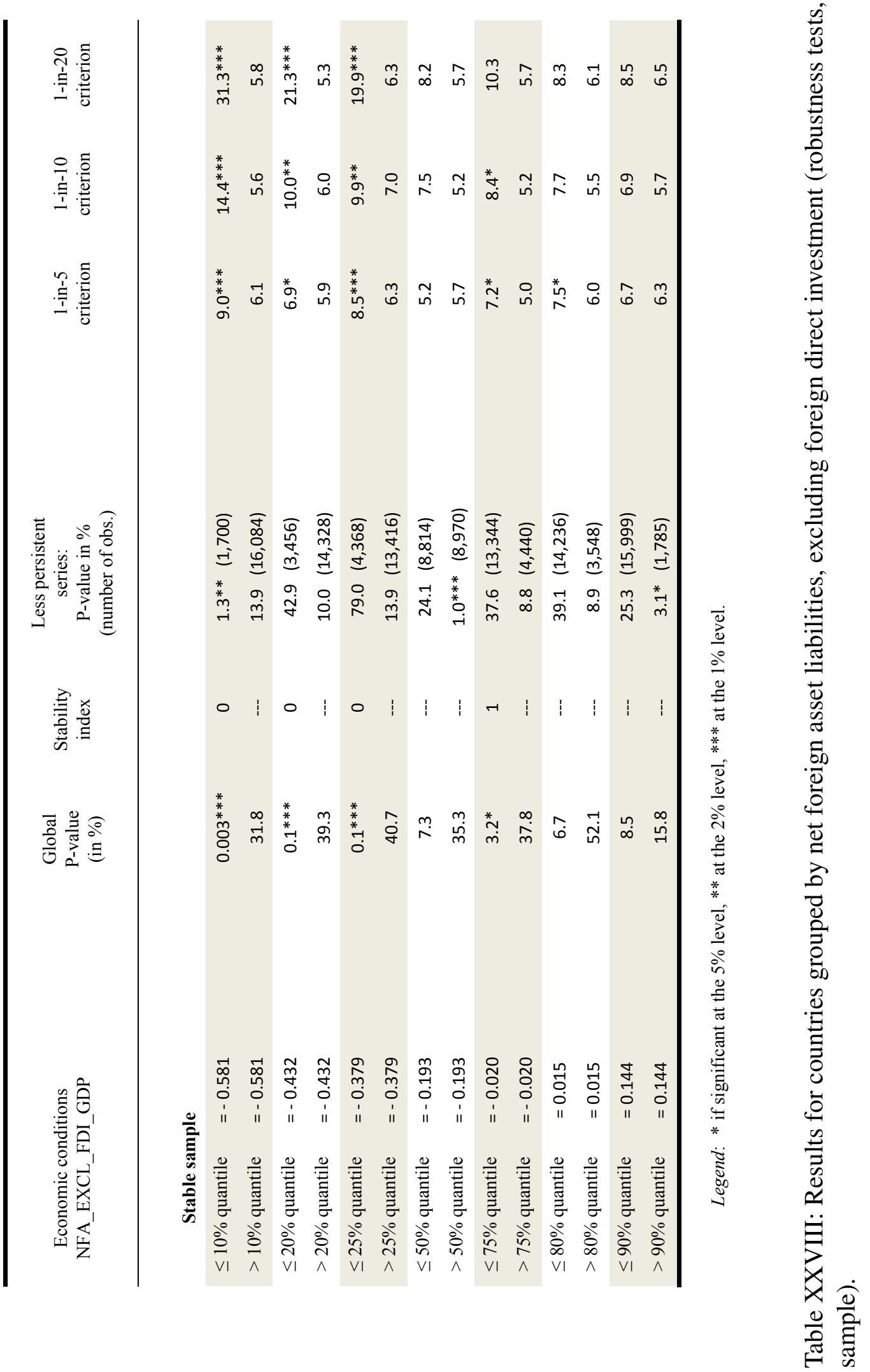




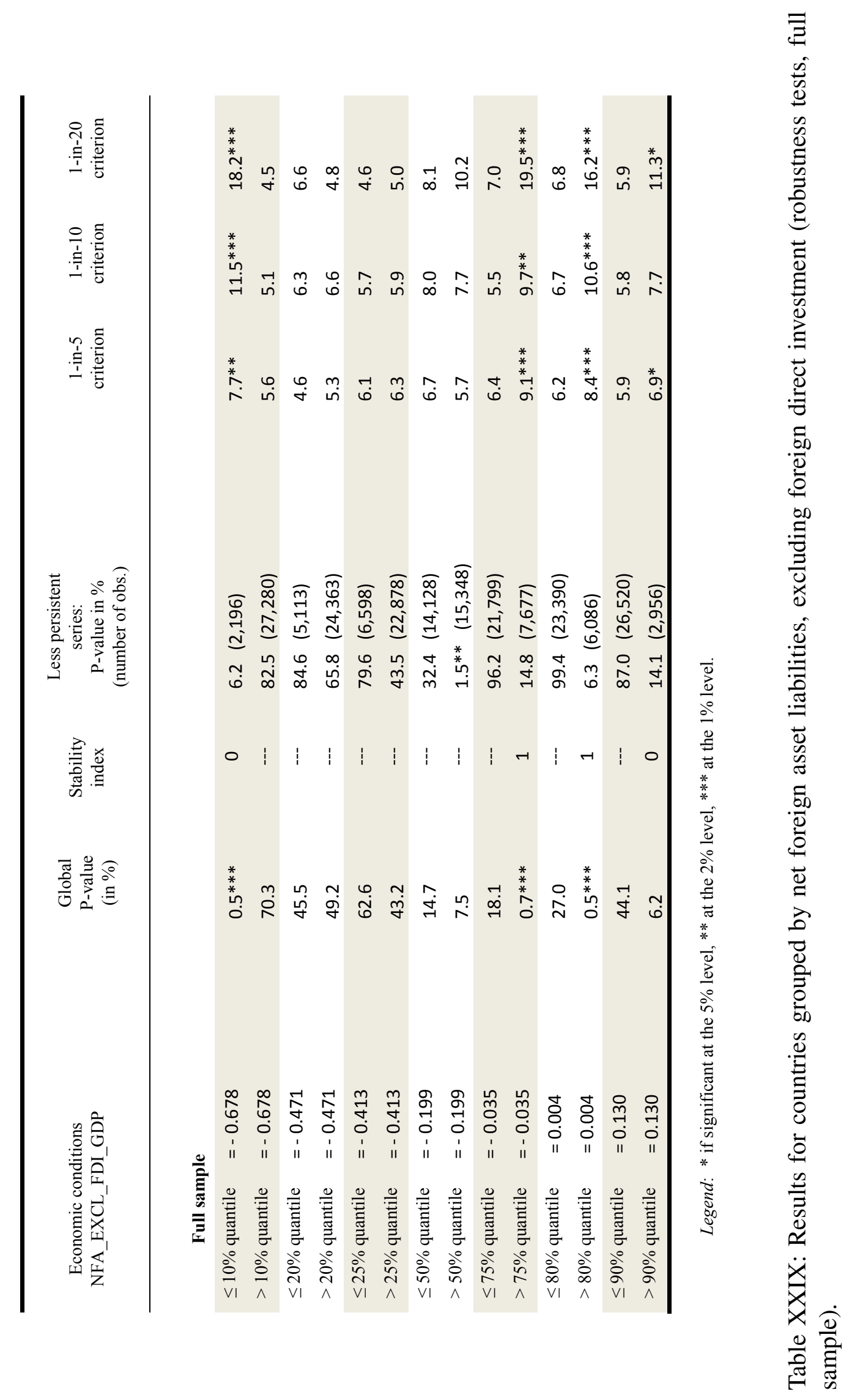




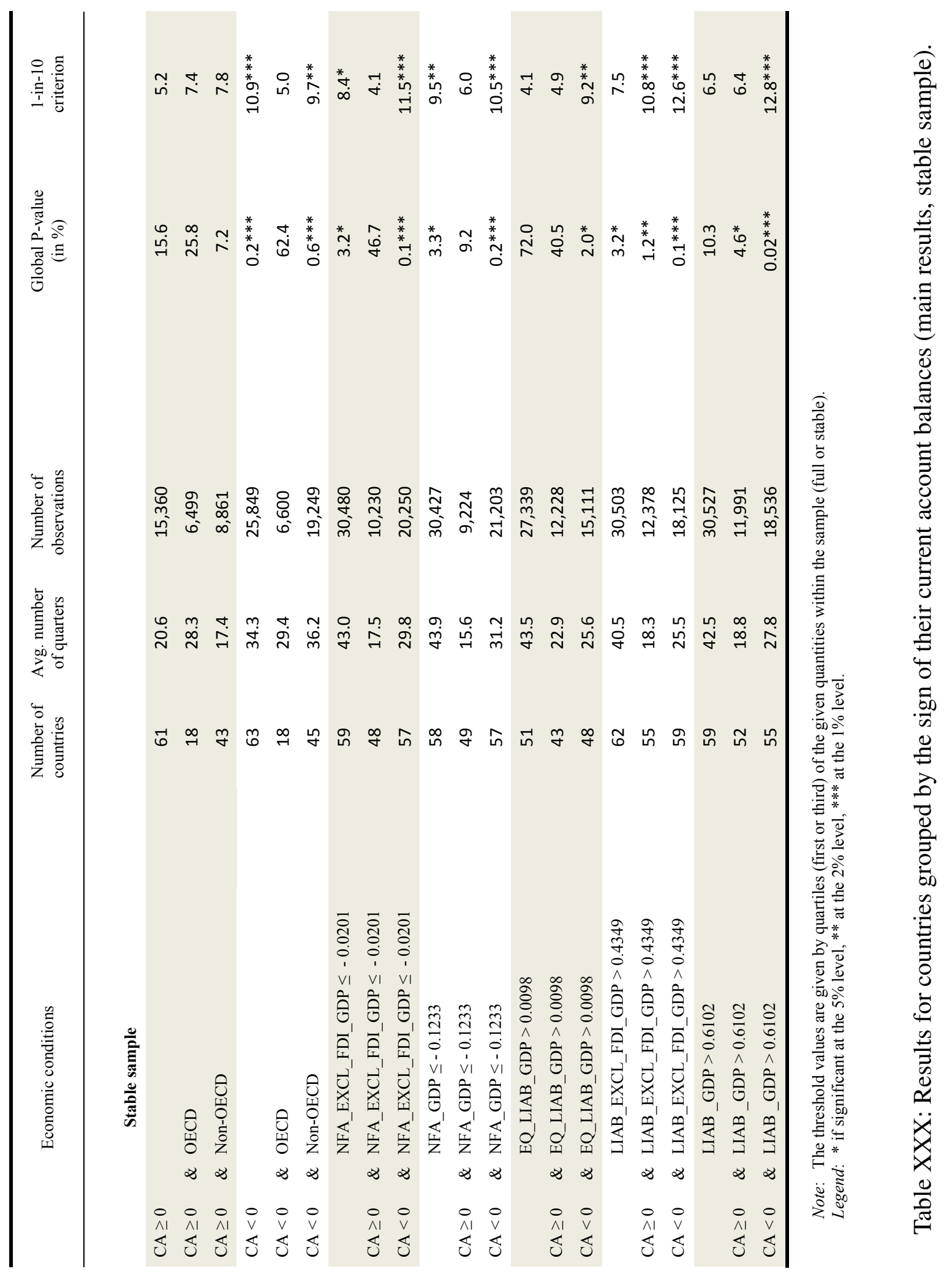




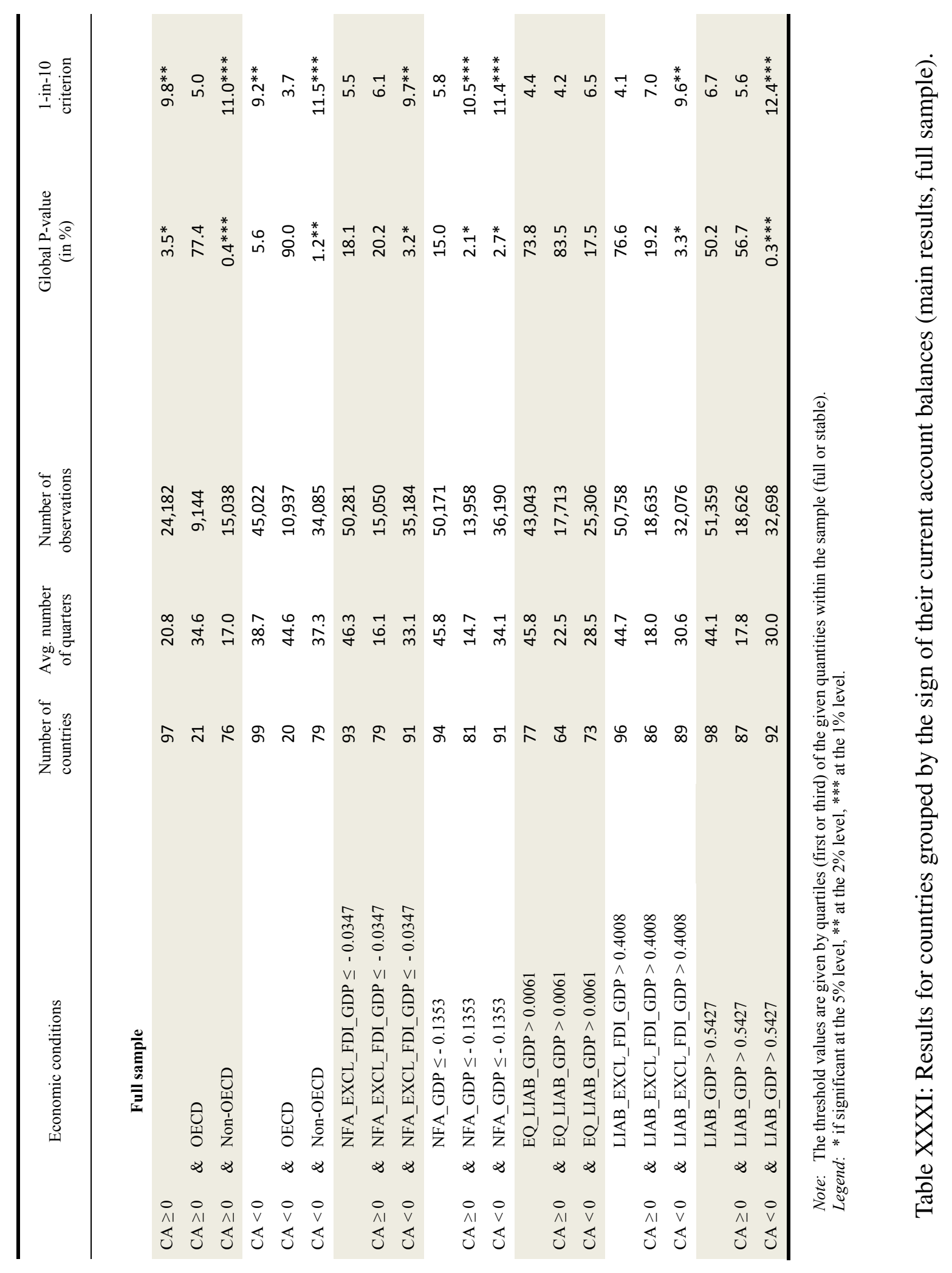




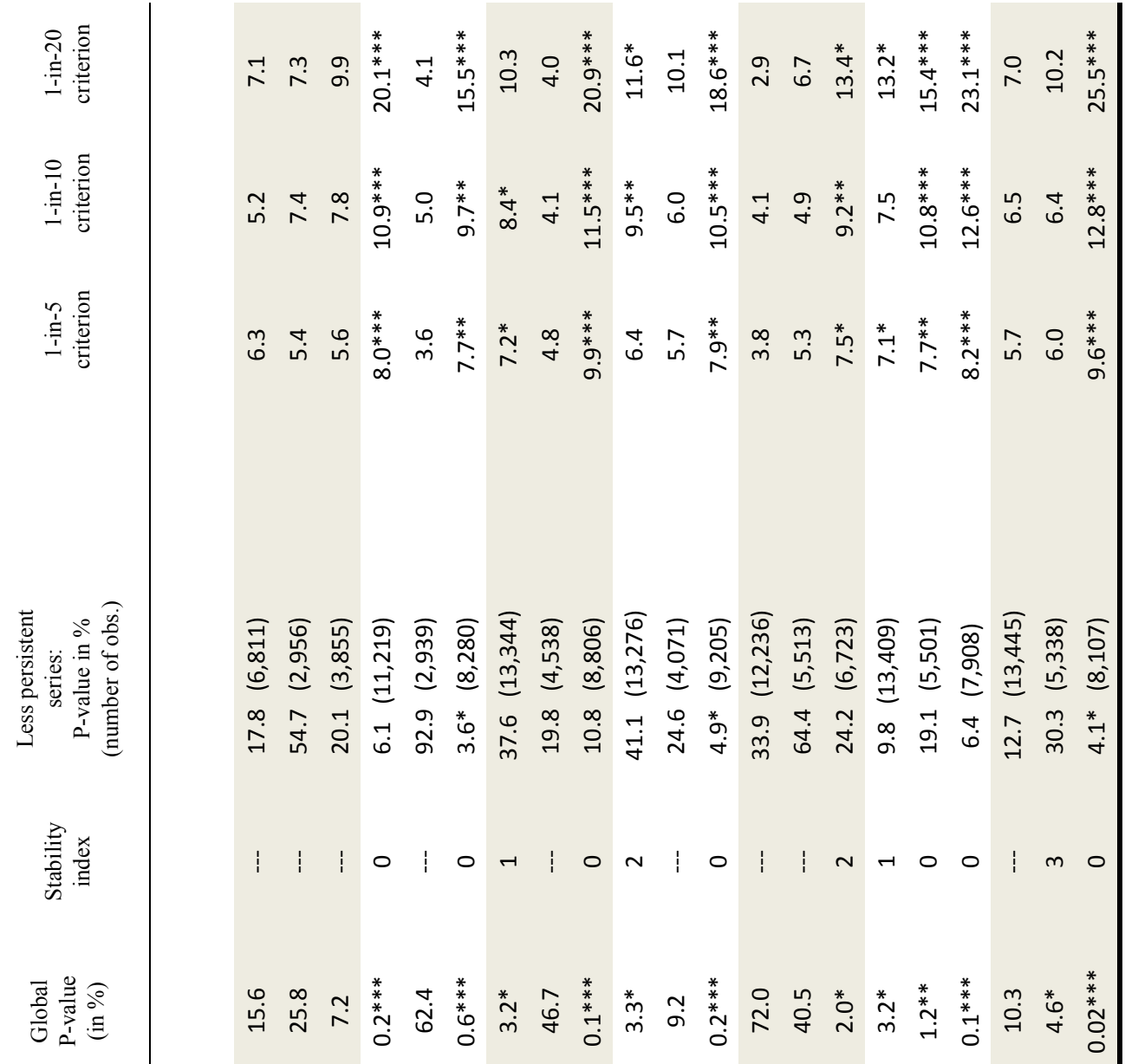

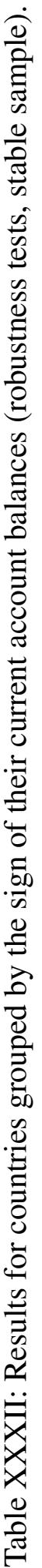




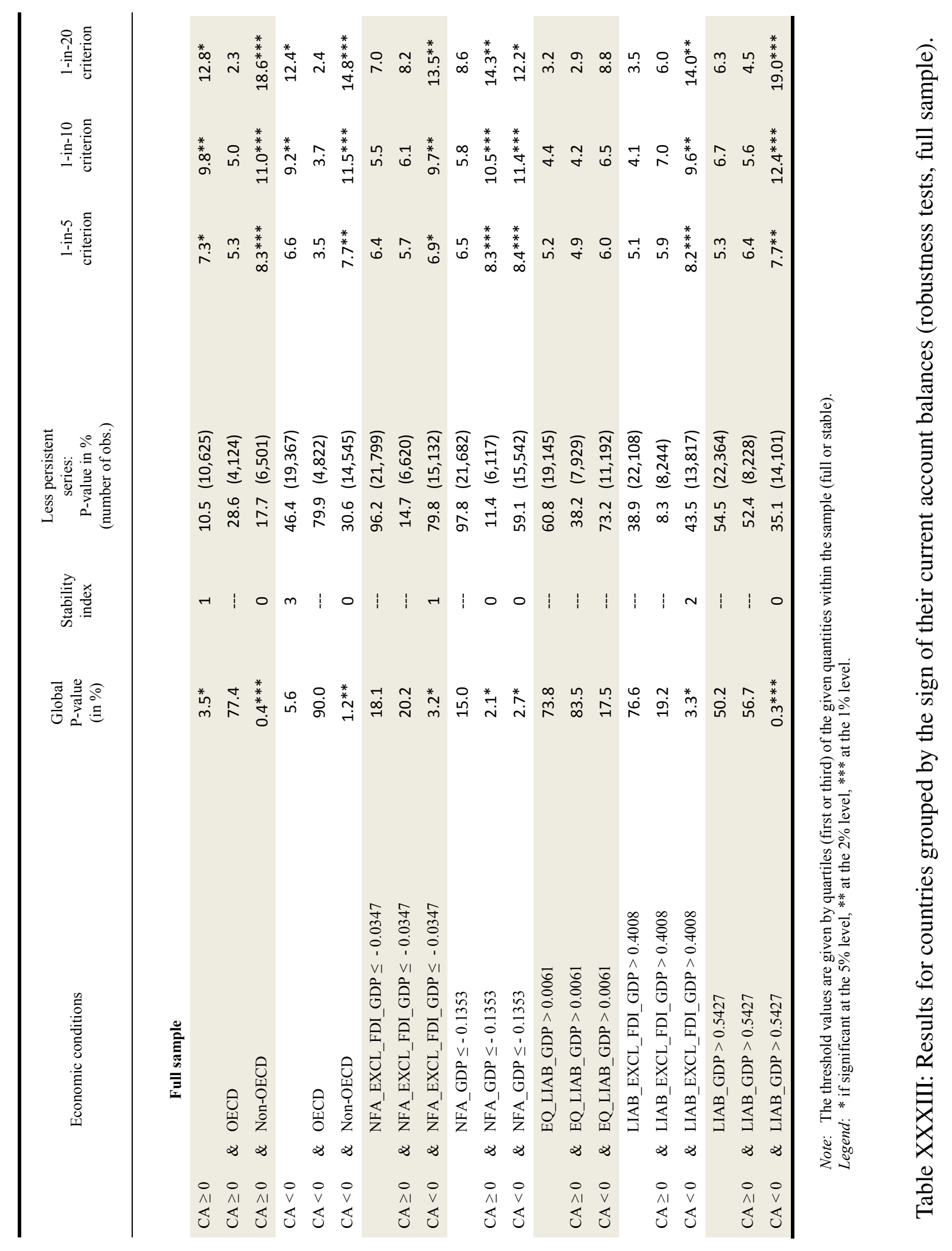




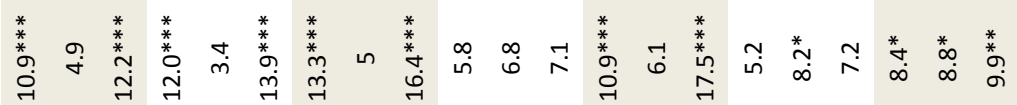

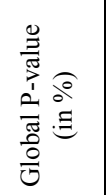

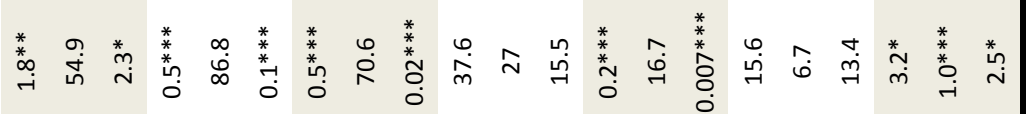

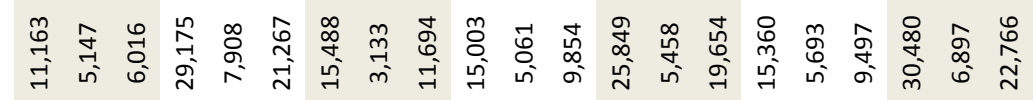

N

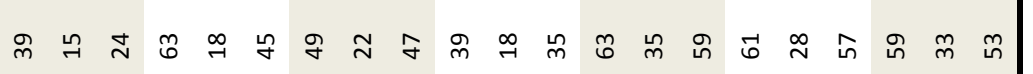

กี

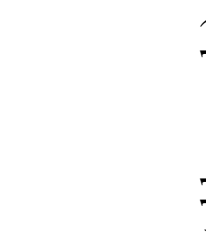

क)

。

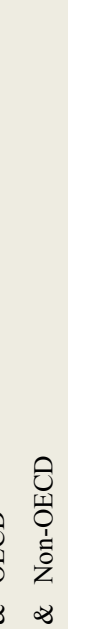$$
+2
$$ 


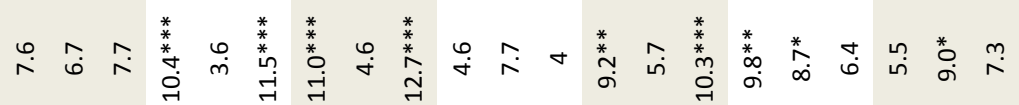

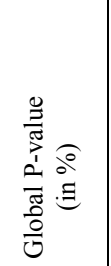

六

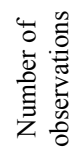

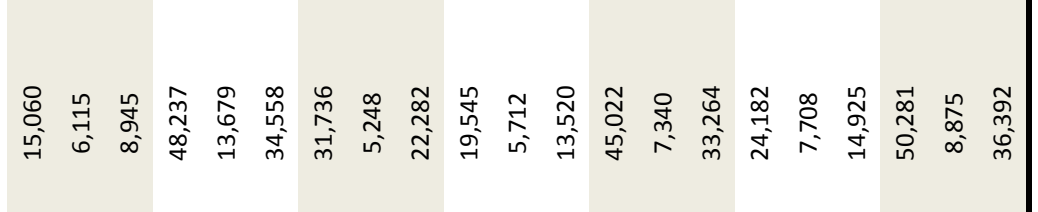

峁

灵

$\sum_{4}^{\infty} \frac{1}{4}$

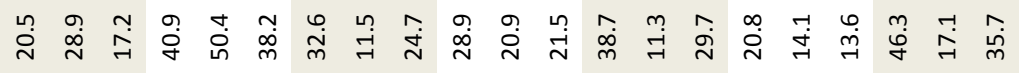

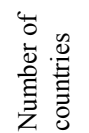

ปี ป

4

(1)

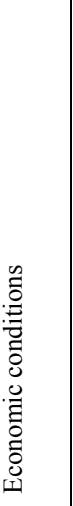

产

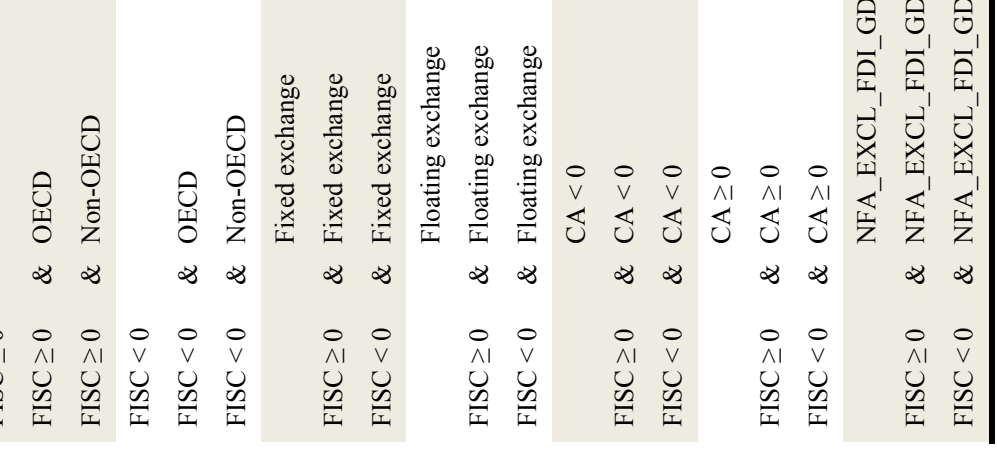

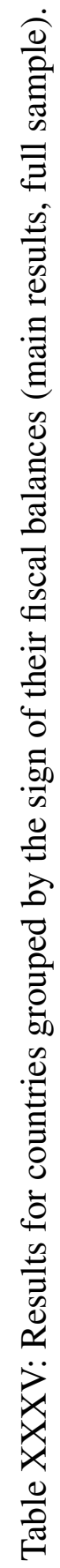




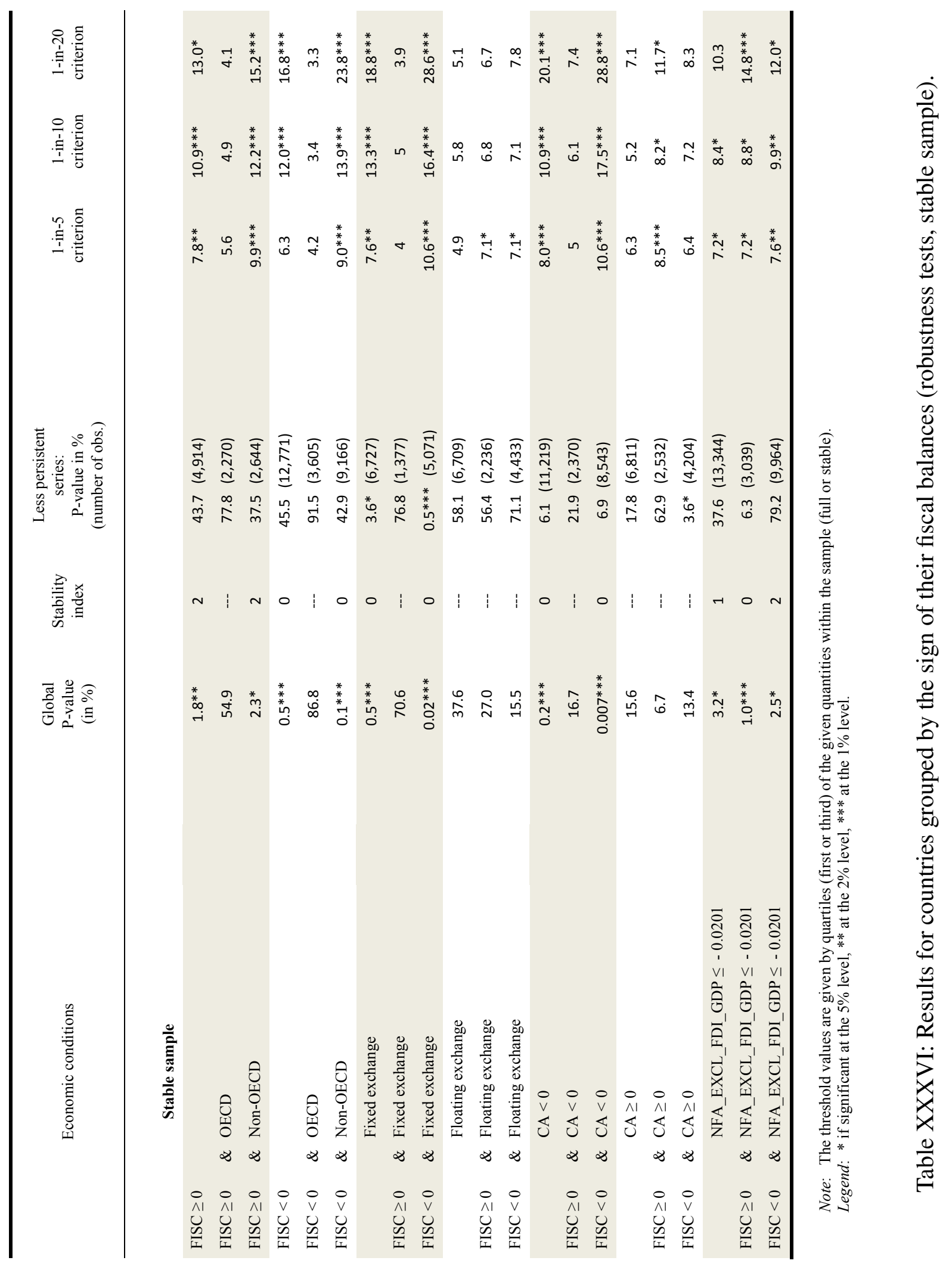




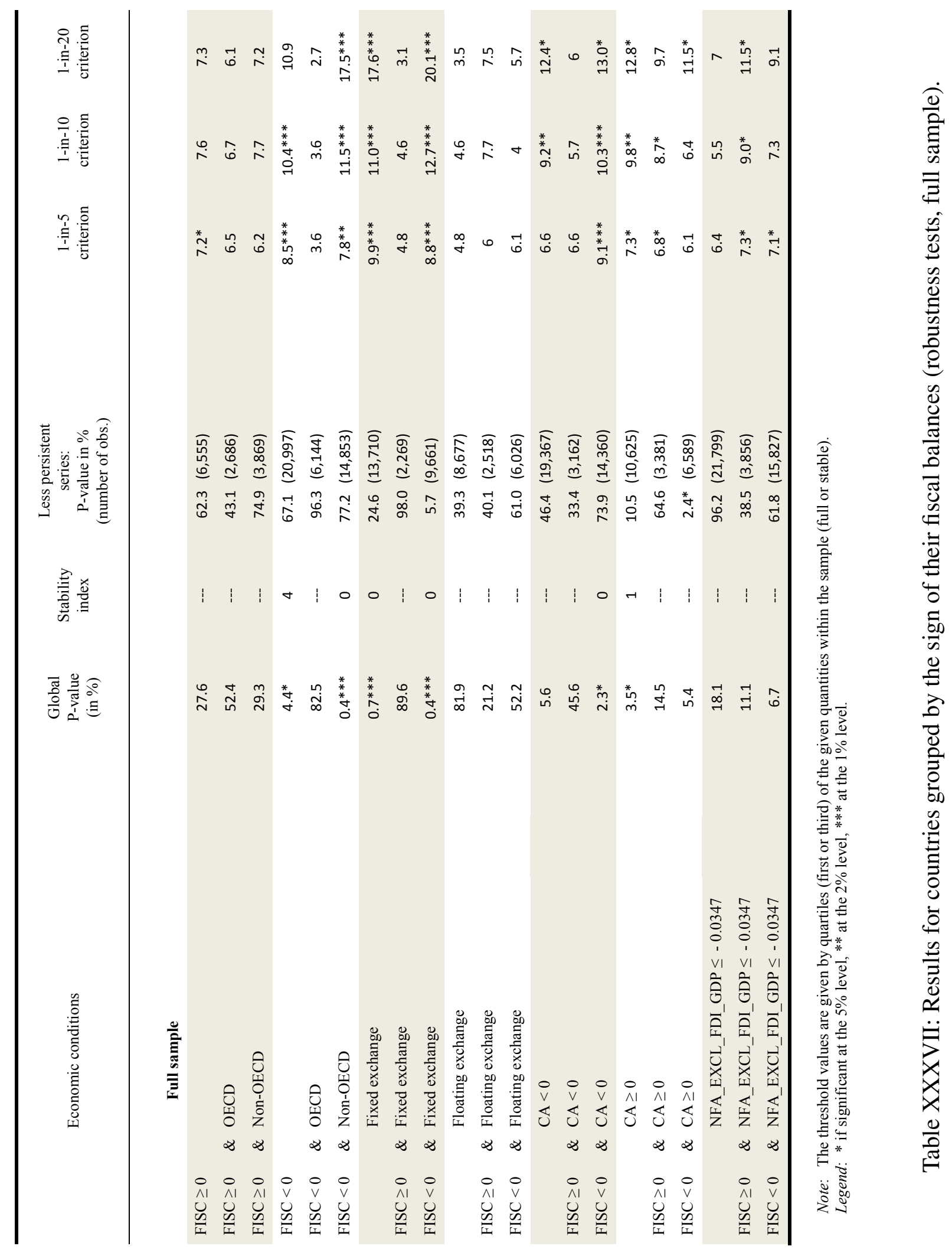




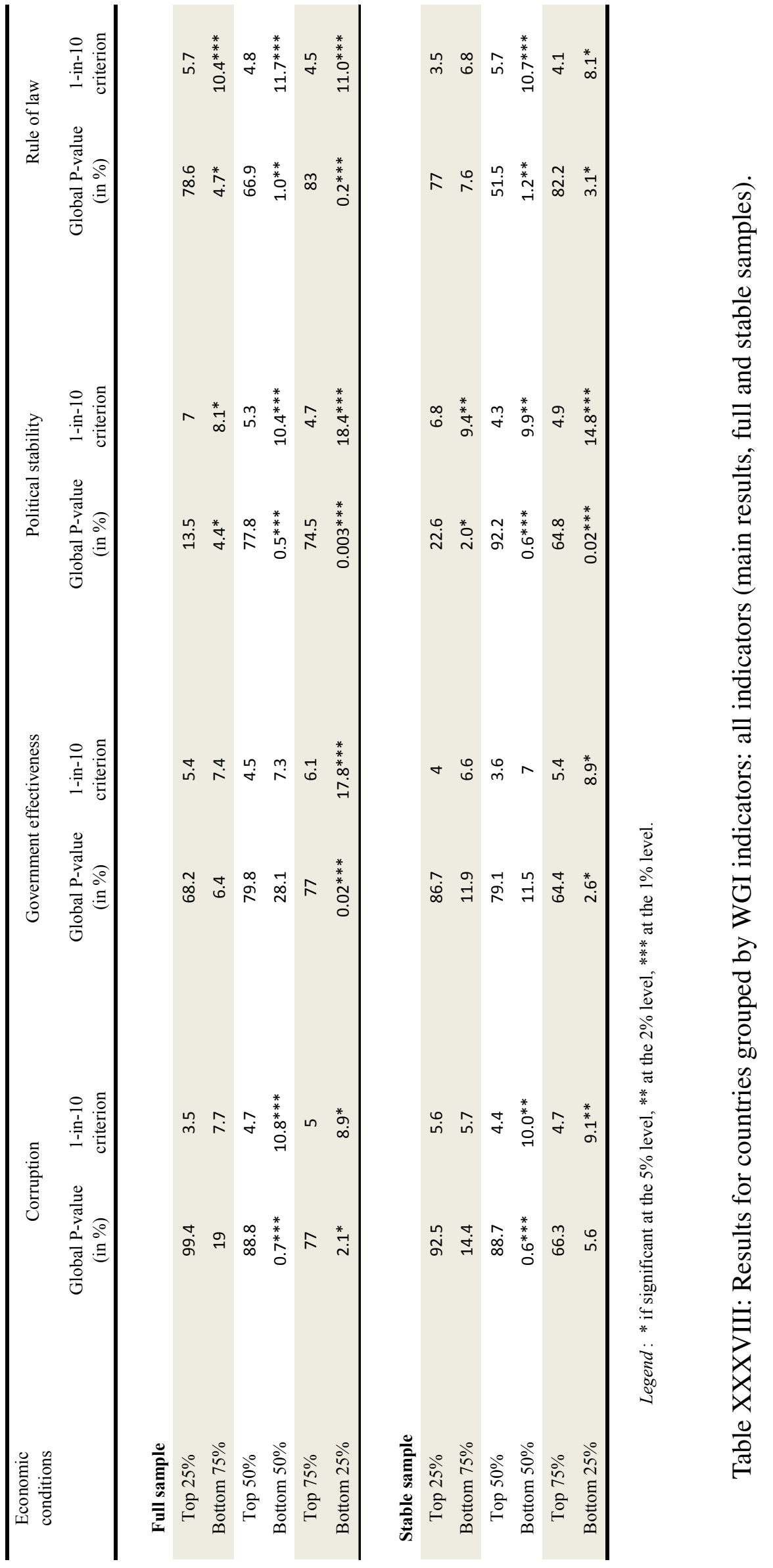




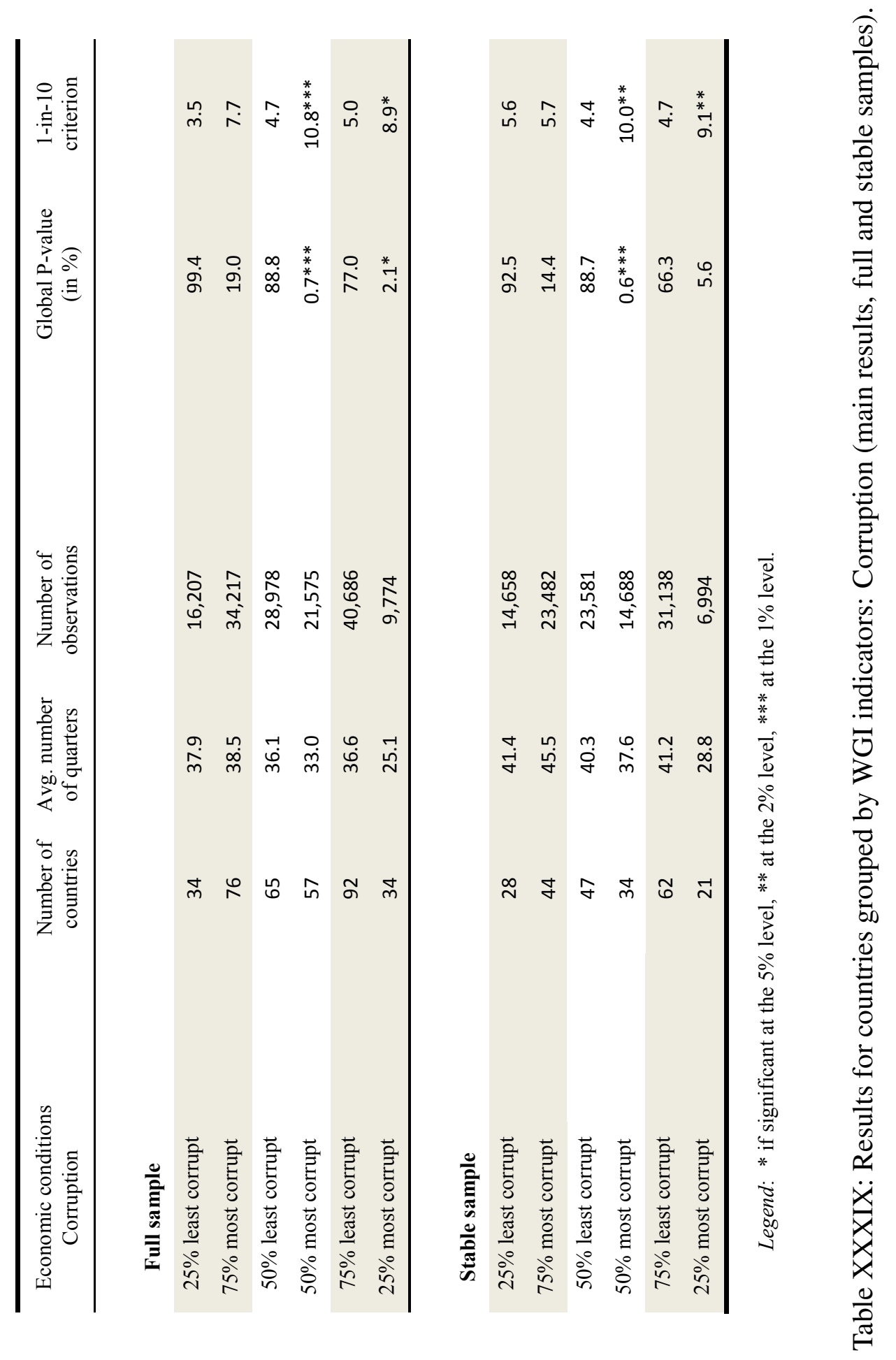




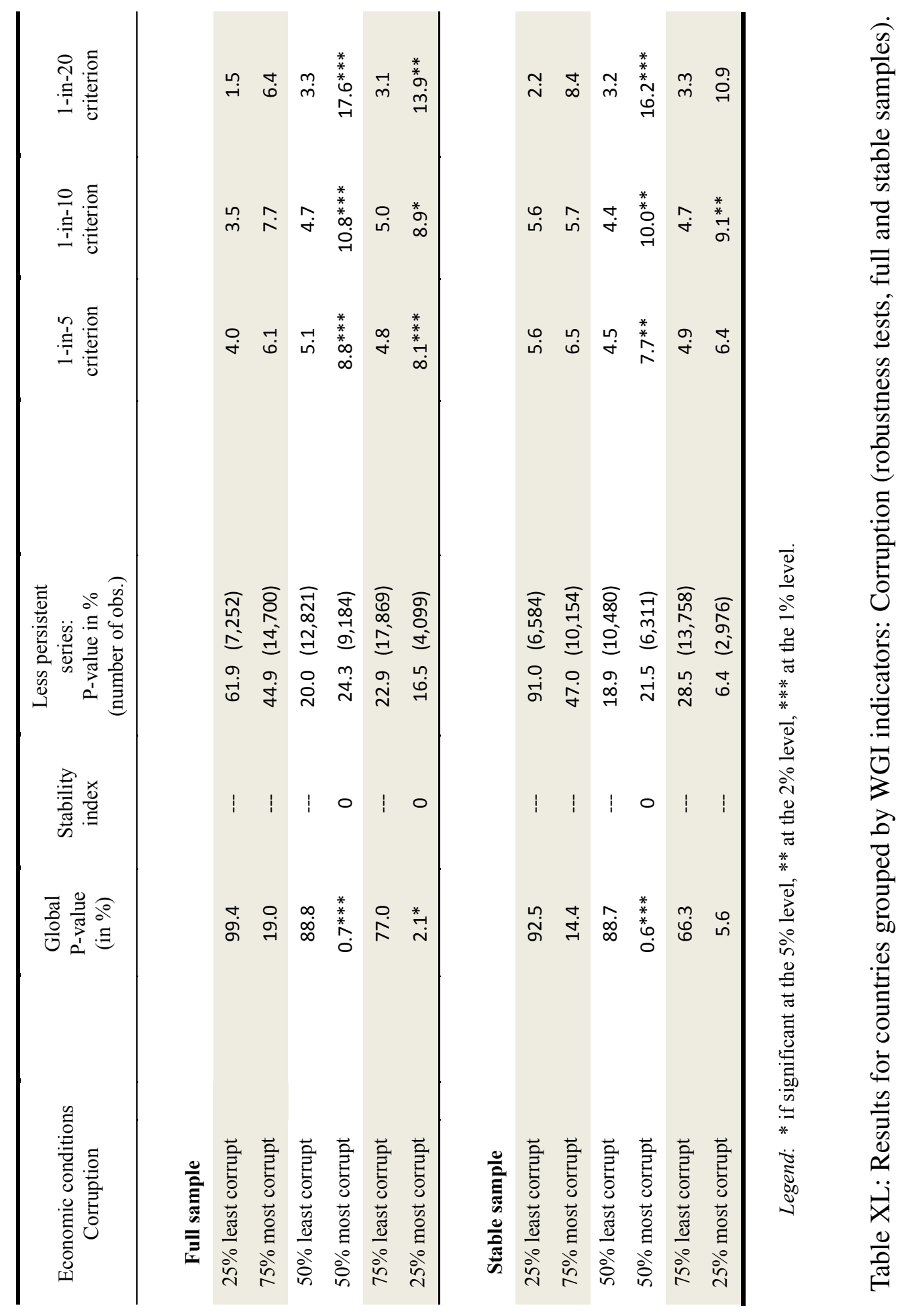




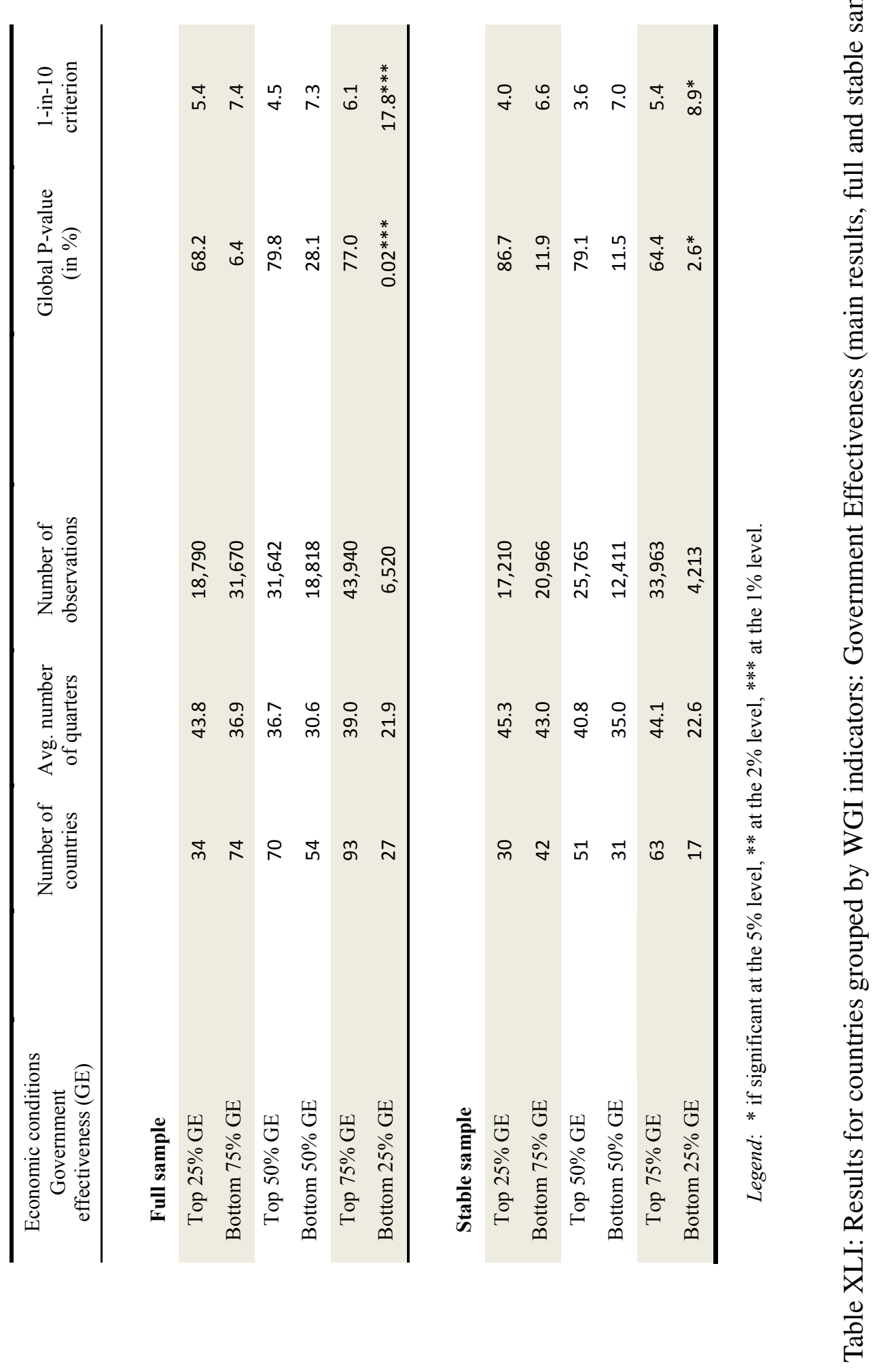




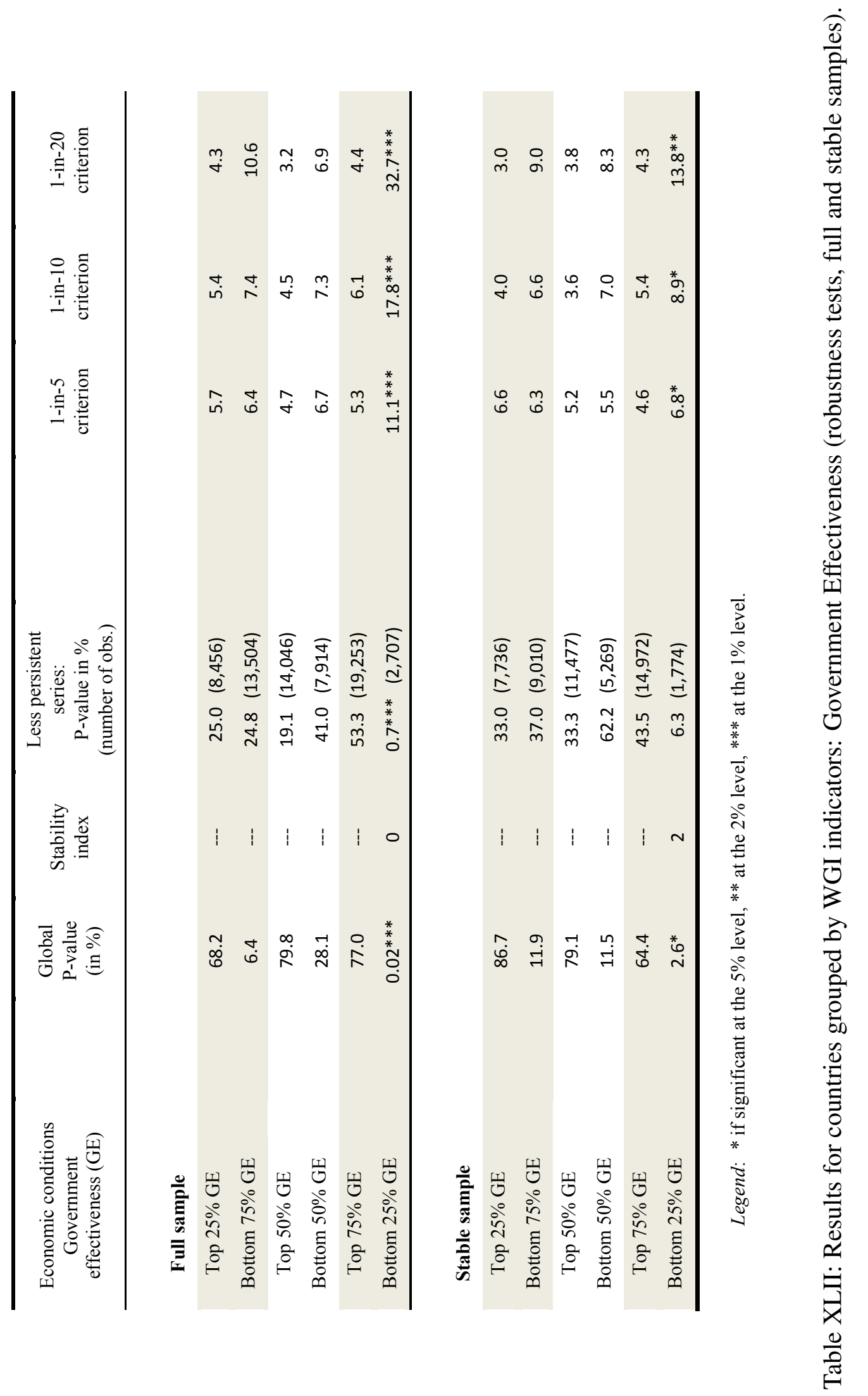




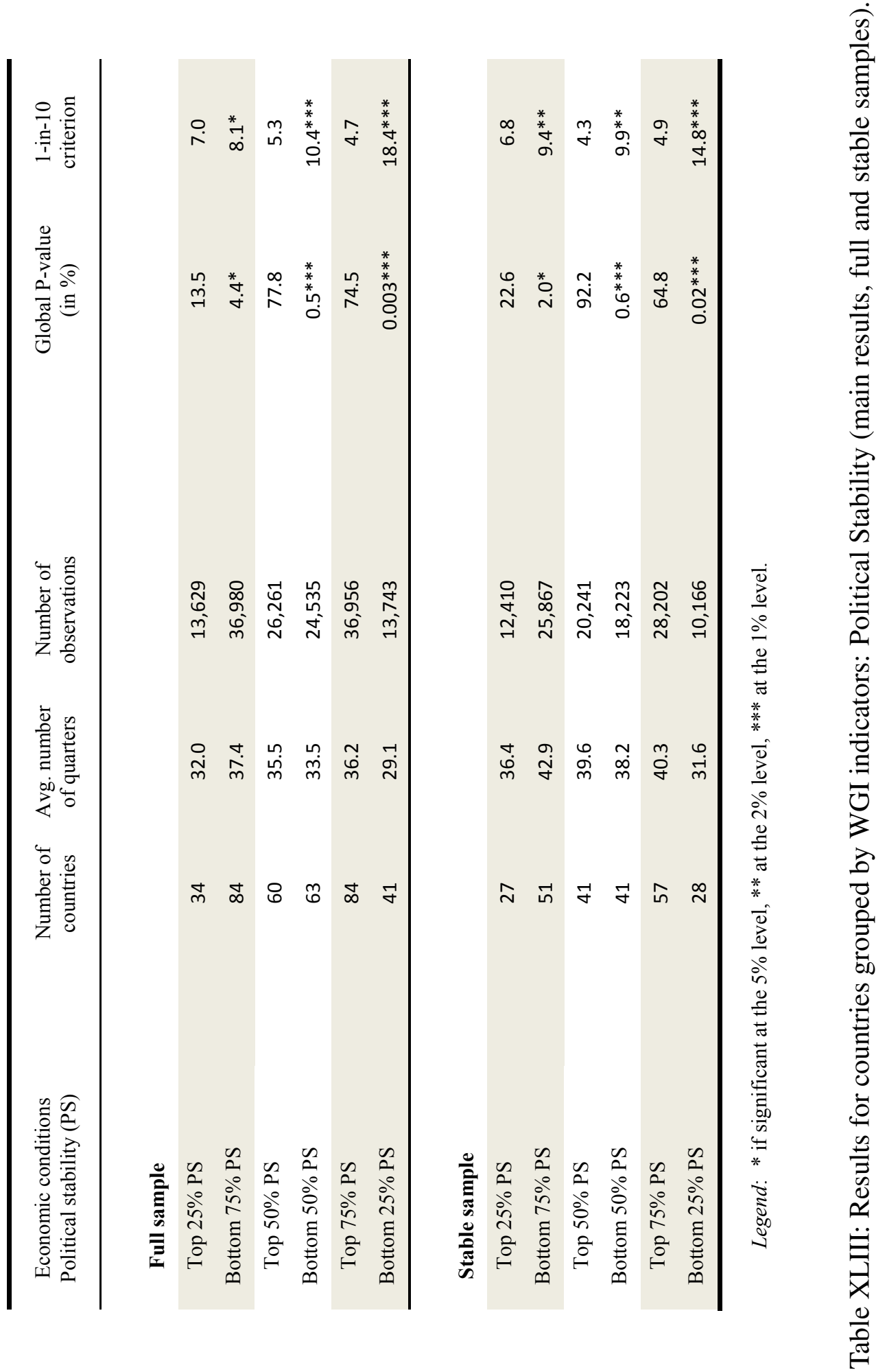




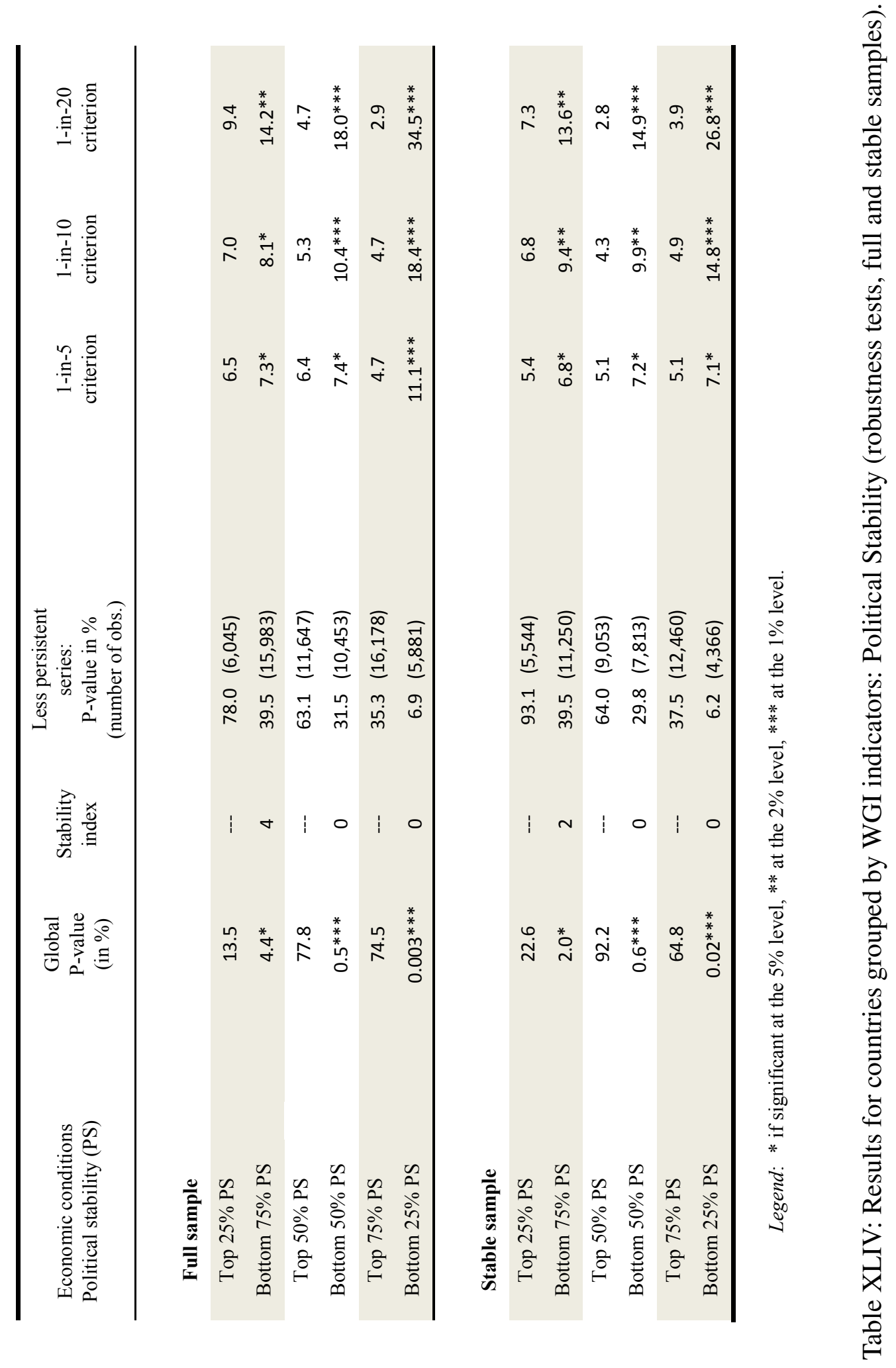




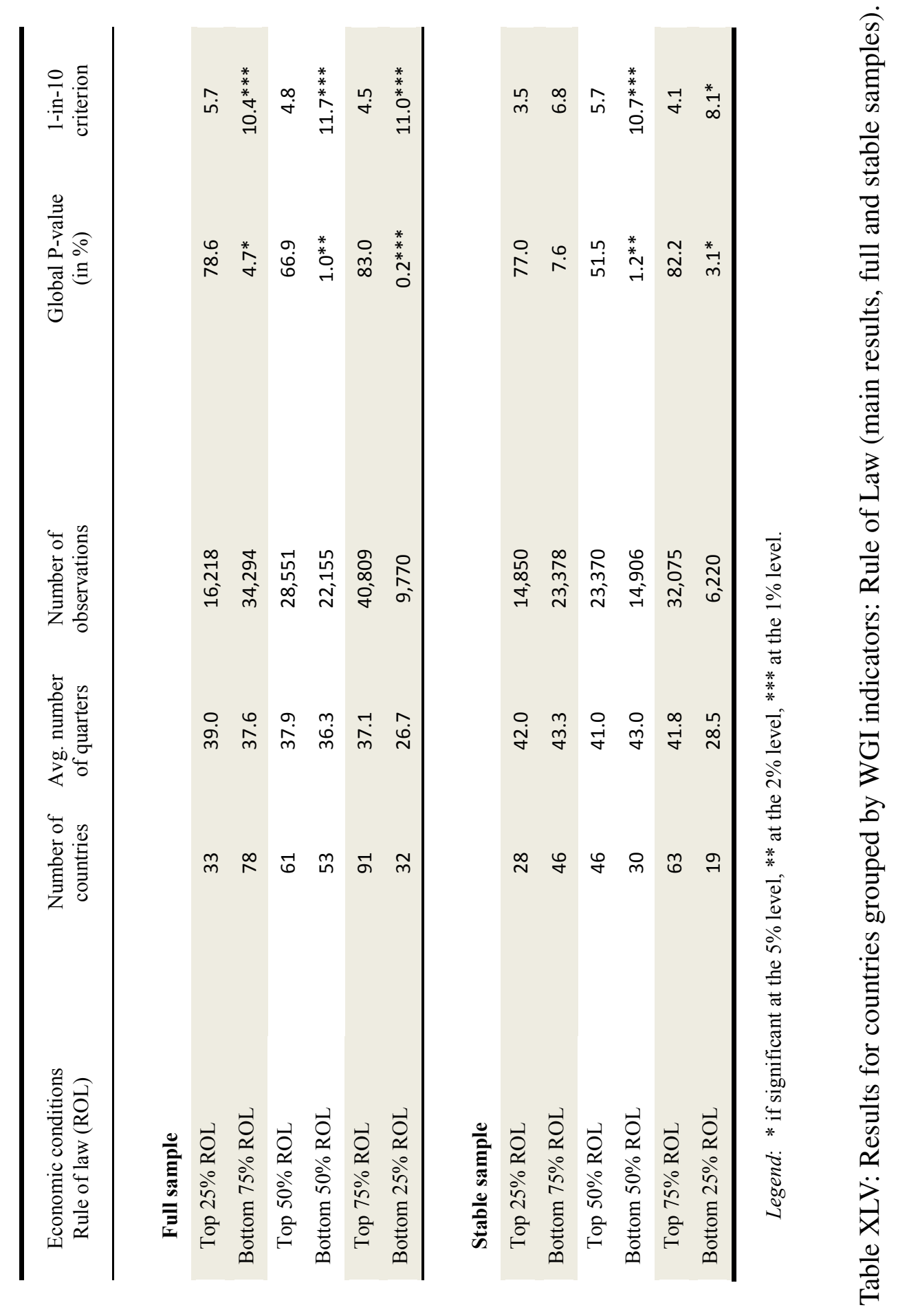




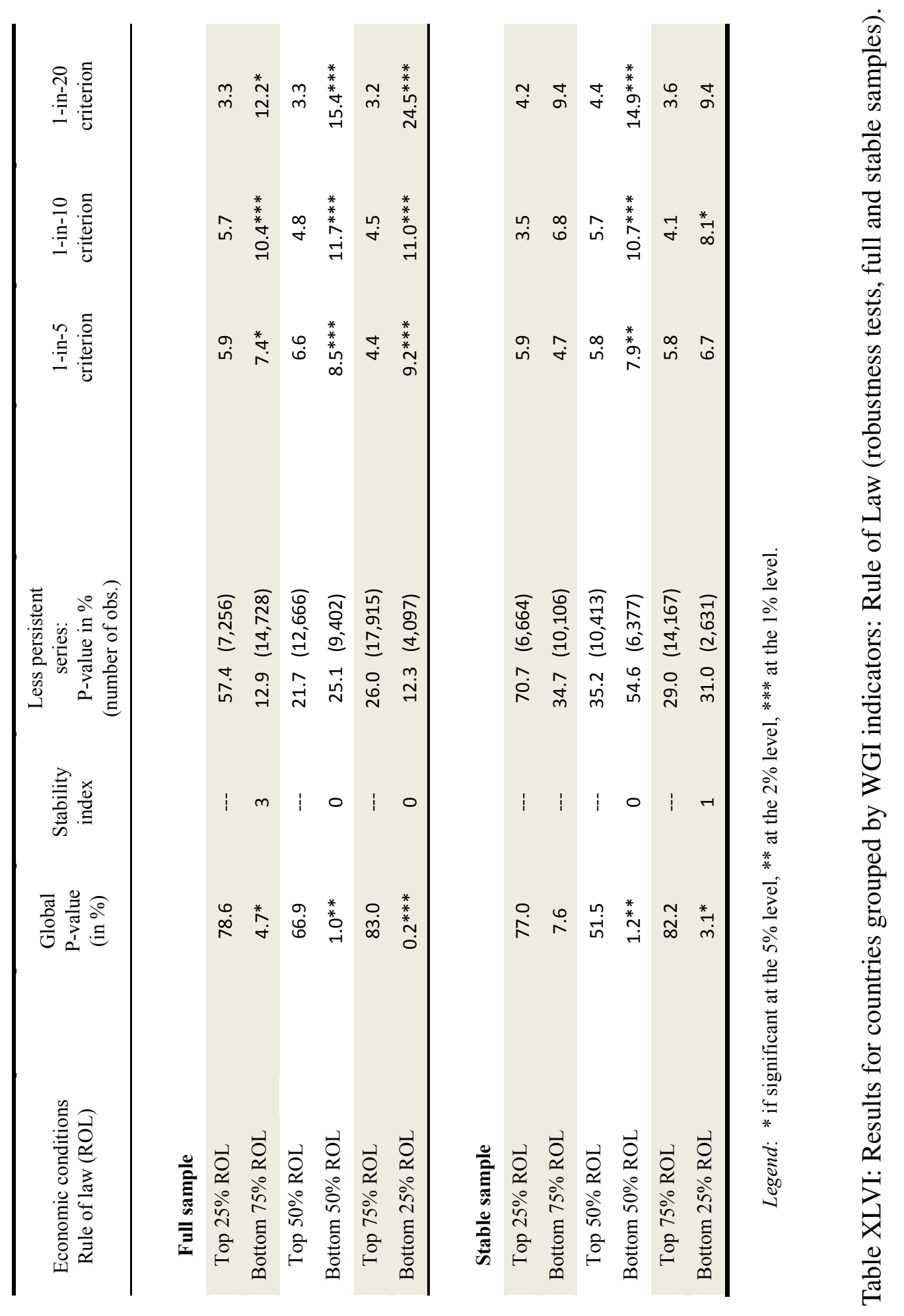



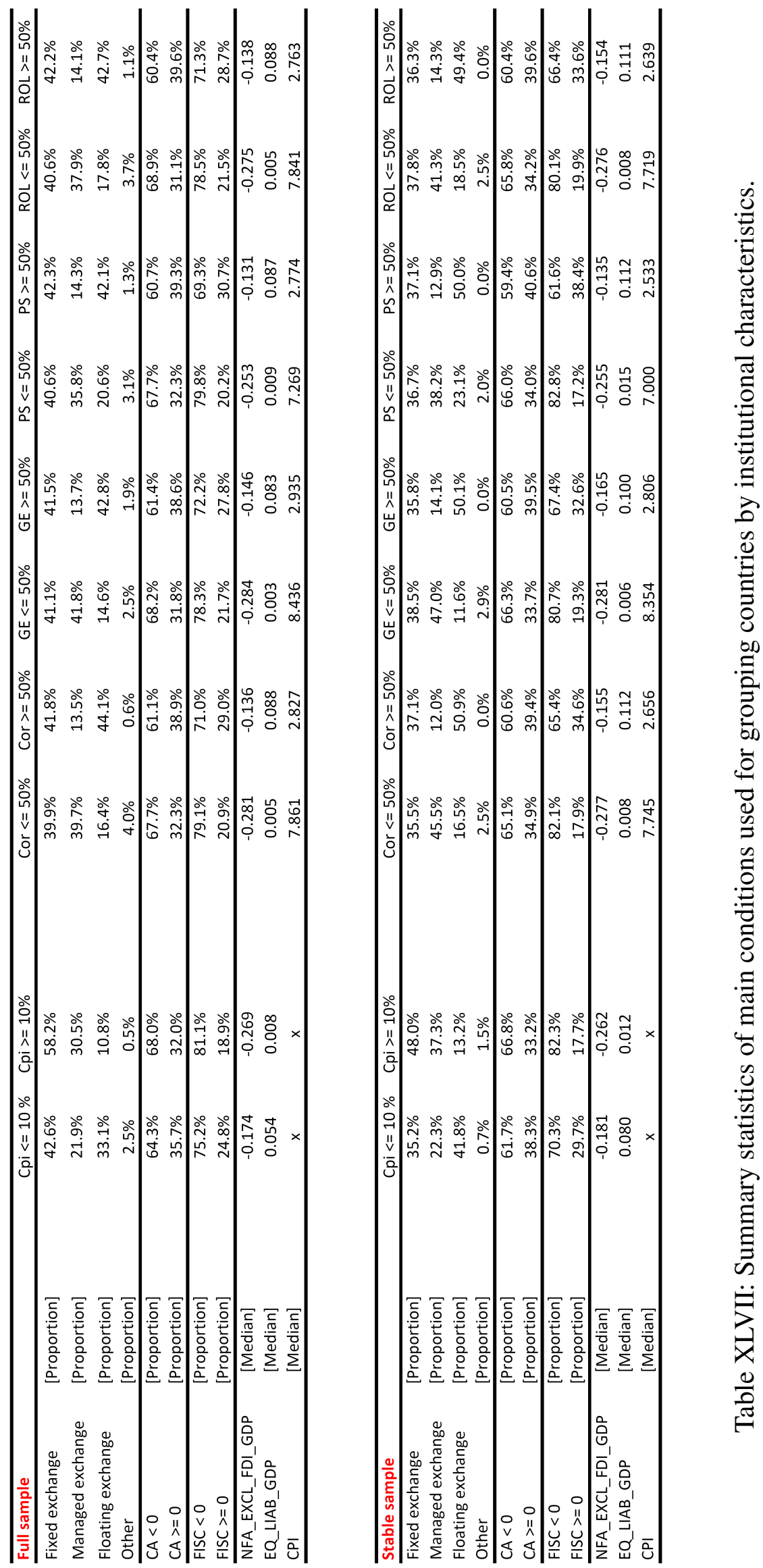


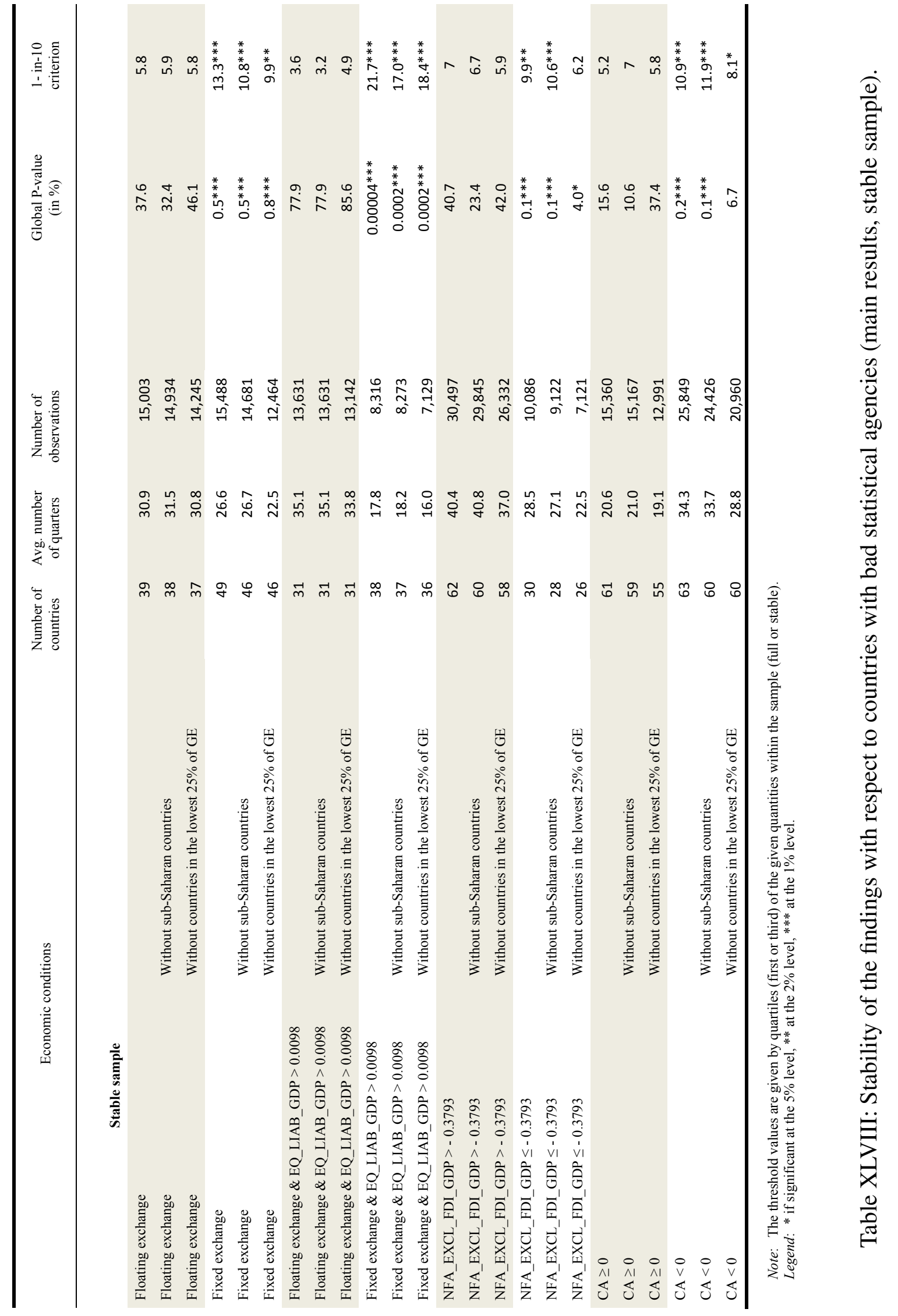




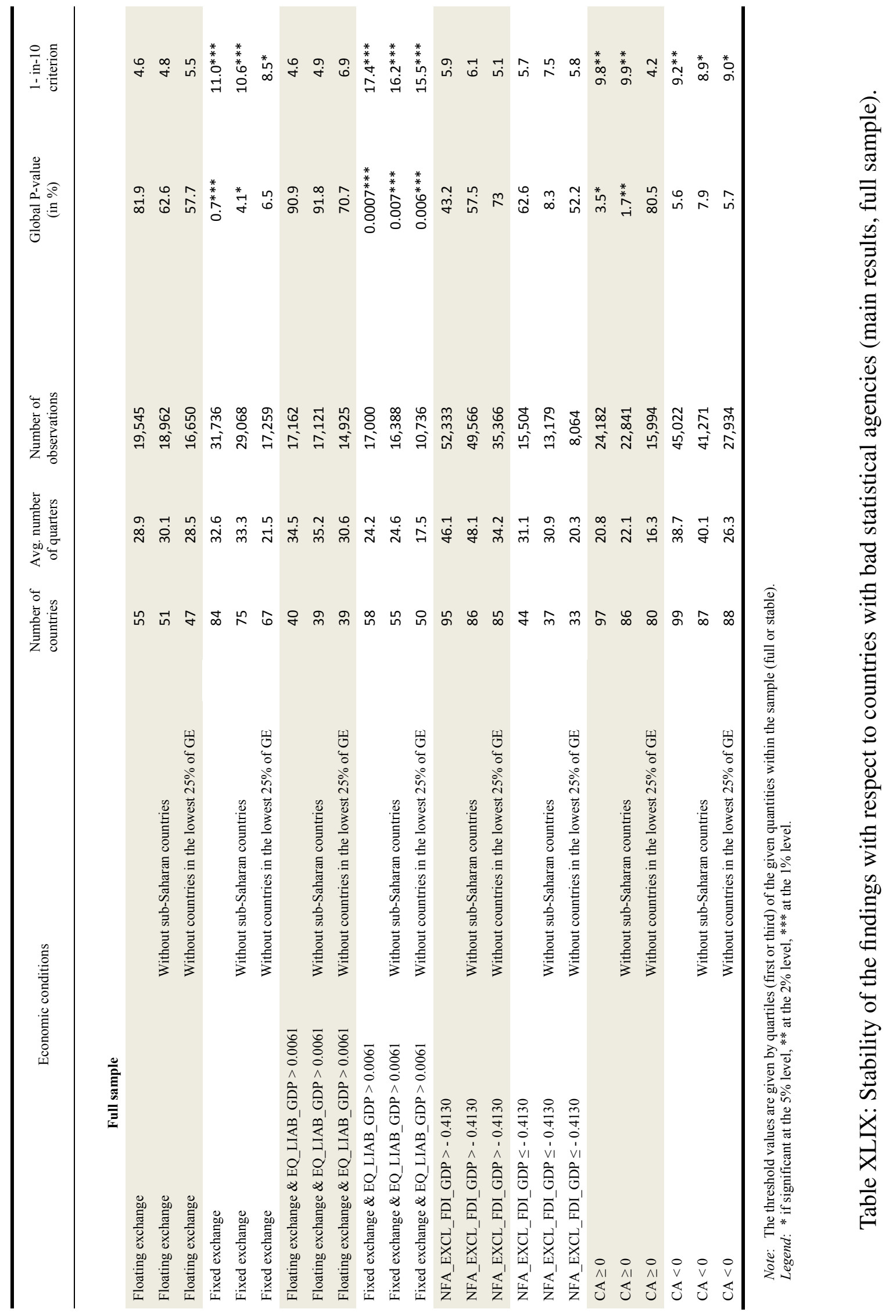




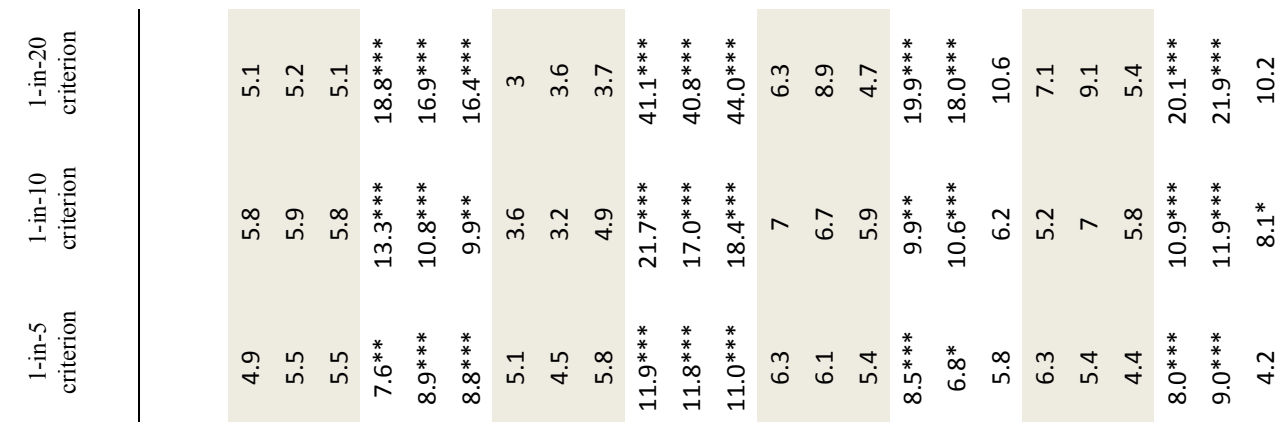

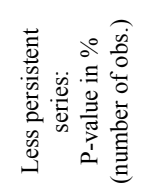

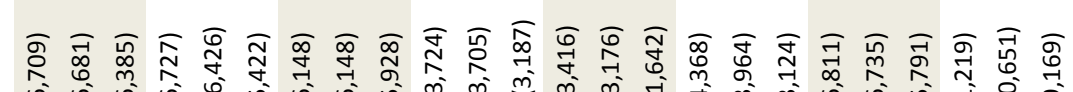
包 南

总总

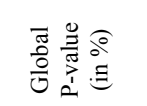
$1: 1000: 1: 000: 1: 000: 11007$

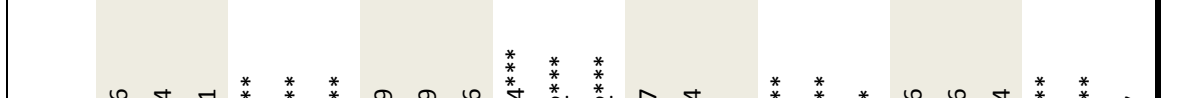

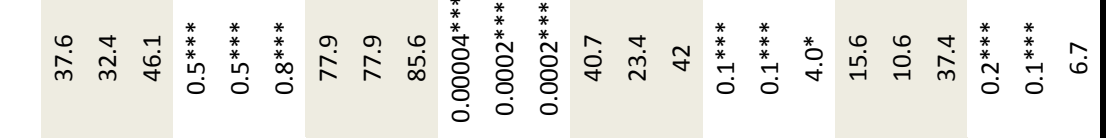
(2)

要

$\frac{1}{2}$

के

葍

$\frac{\mathscr{0}}{0}$

必

:

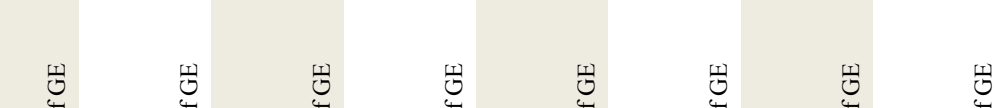

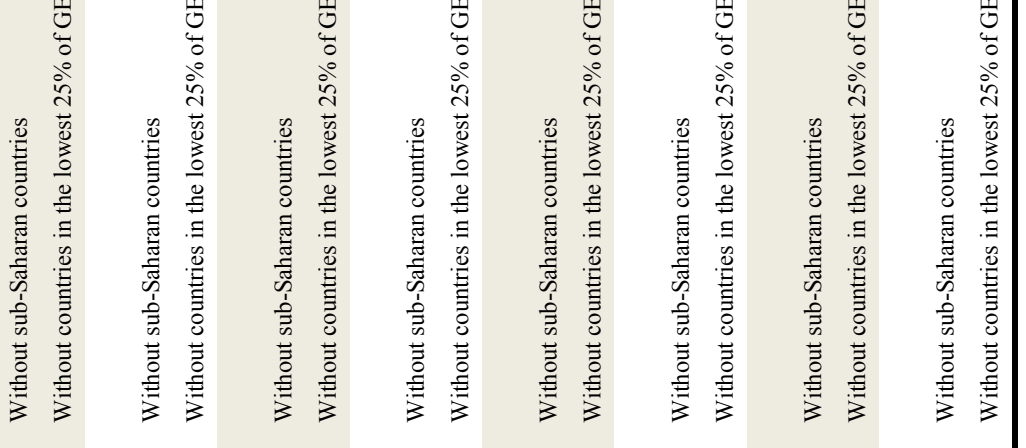

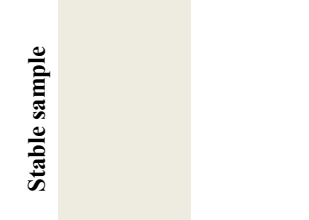

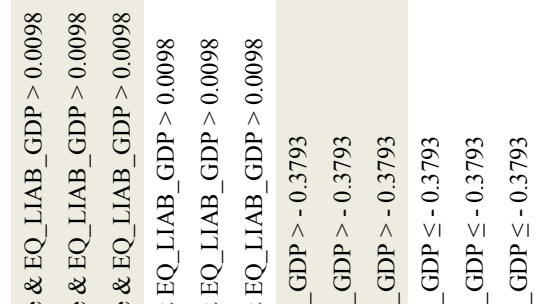

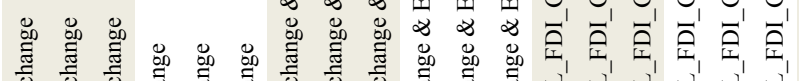




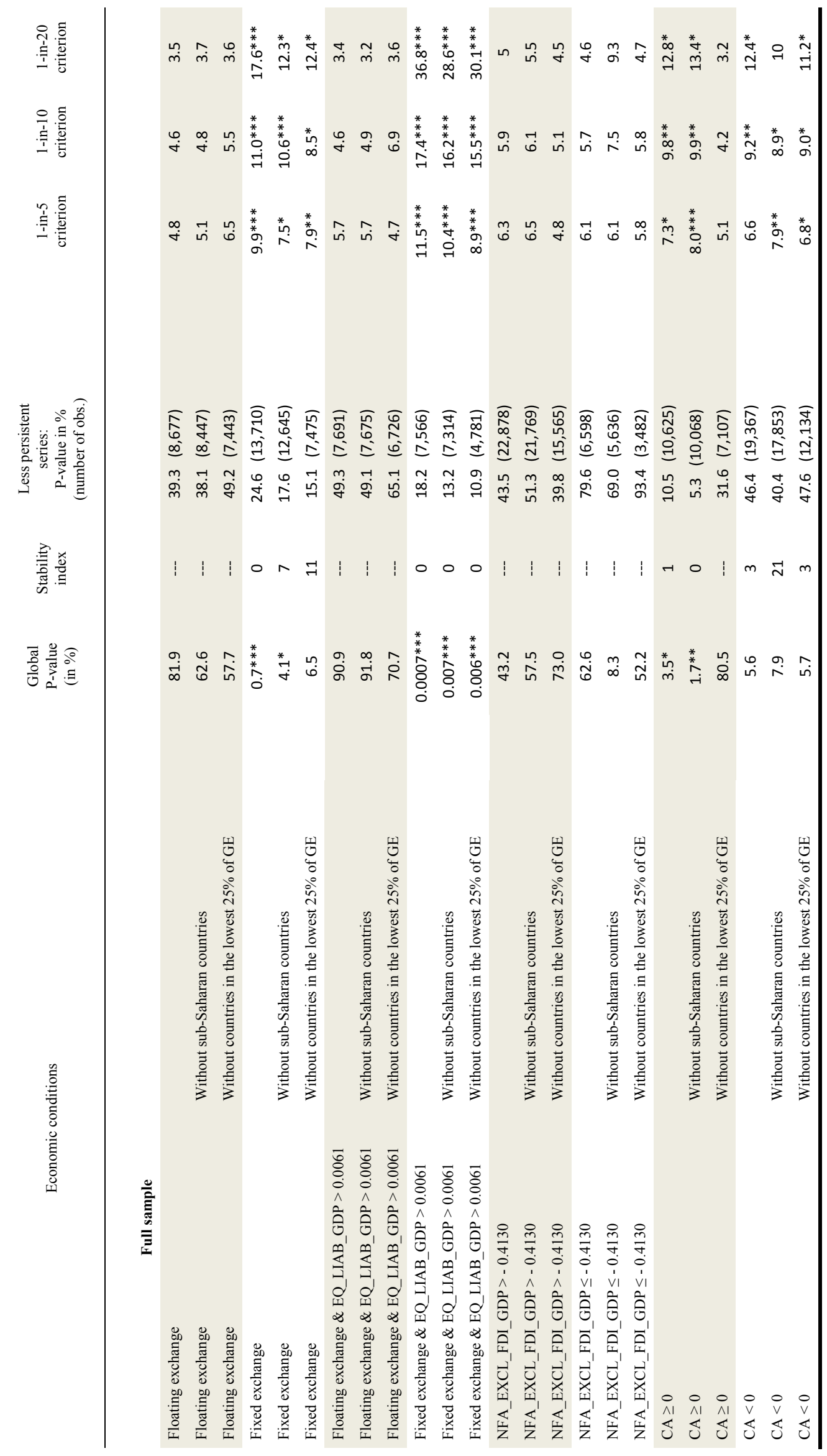

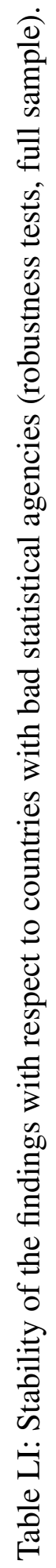




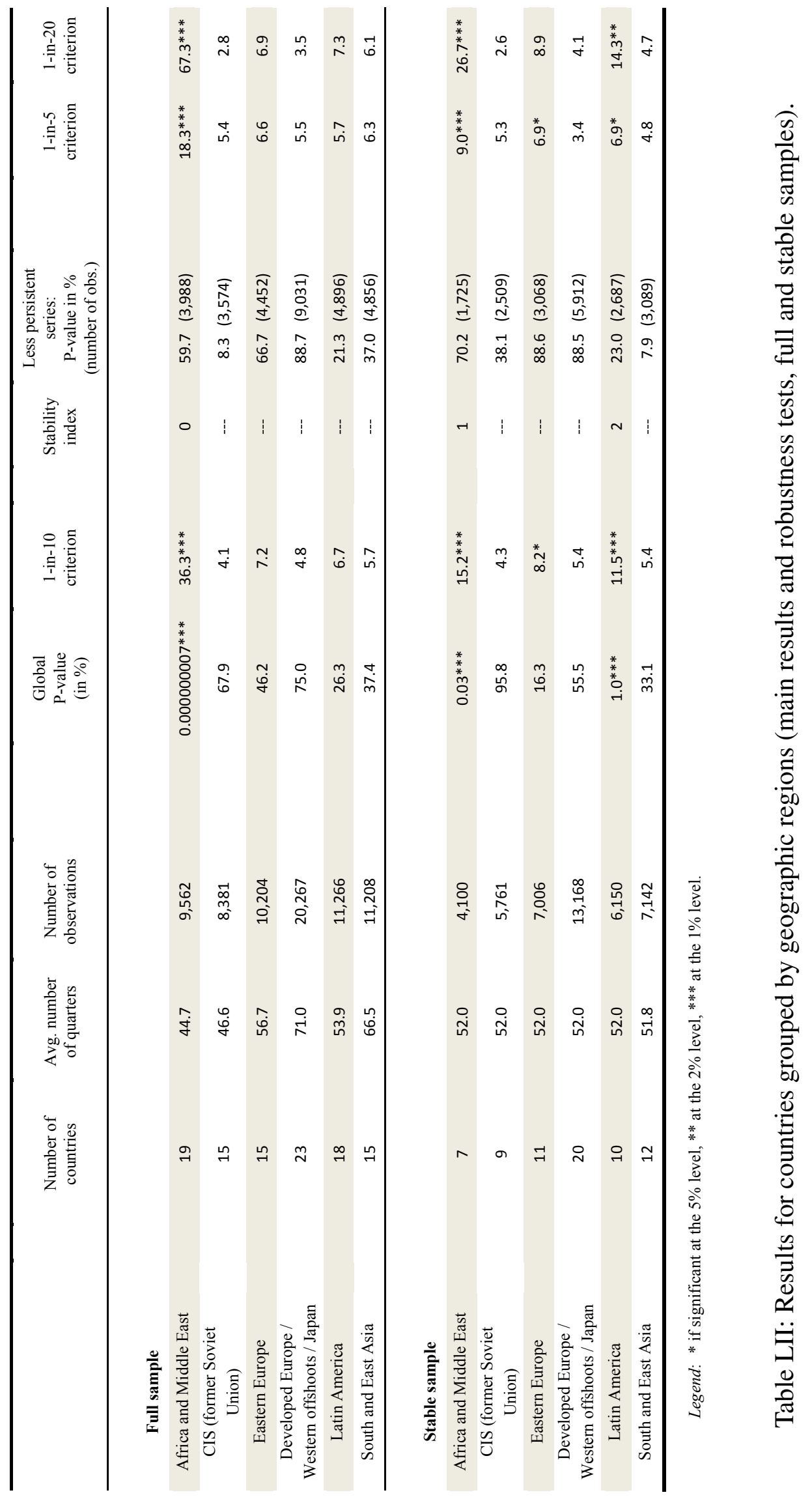




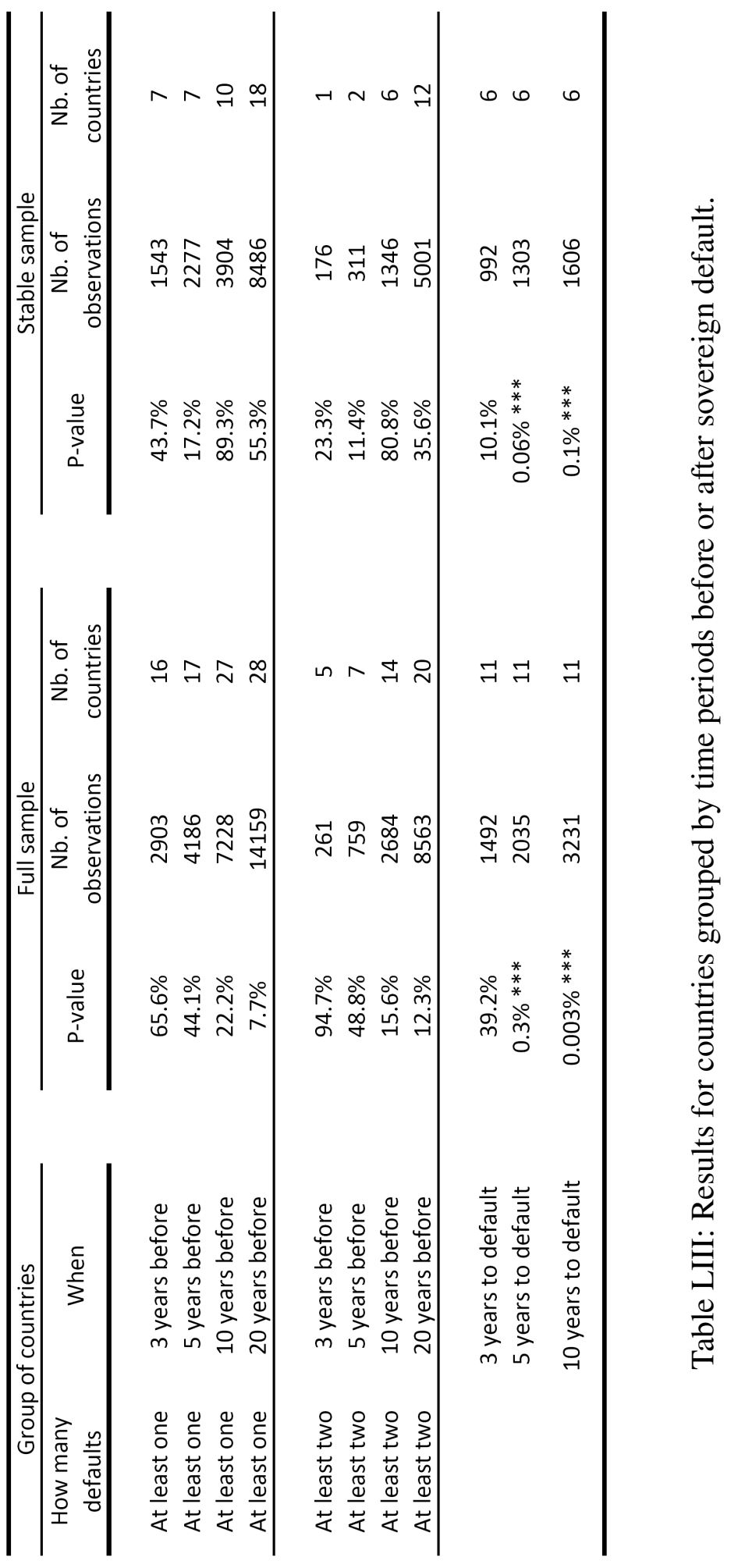




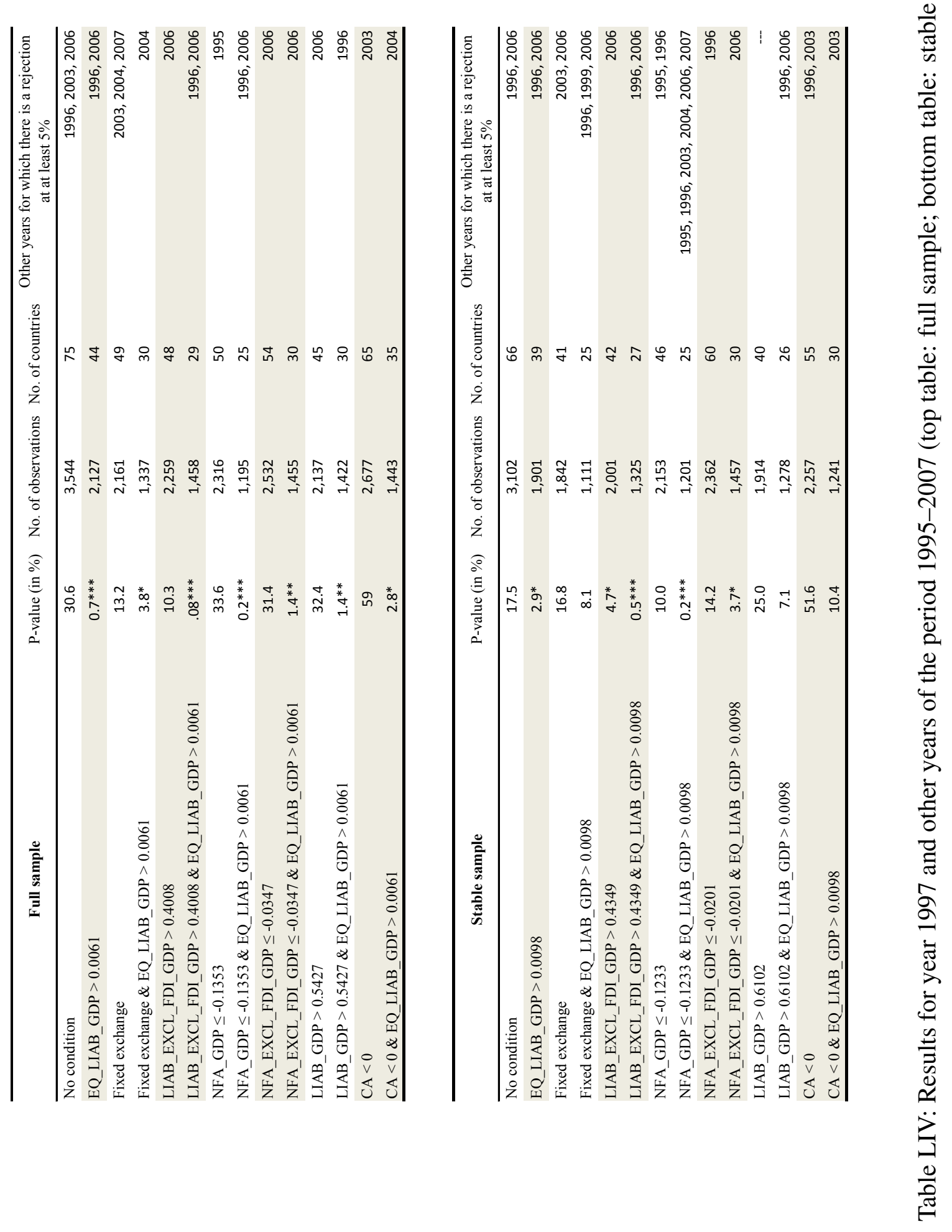

